\title{
Wave condensation with weak disorder vs beam self-cleaning in multimode fibers
}

\author{
J. Garnier ${ }^{1}$, A. Fusaro ${ }^{2}$, K. Baudin ${ }^{2}$, C. Michel, ${ }^{3}$ K. Krupa ${ }^{2}$, G. Millot ${ }^{2}$, A. Picozzi ${ }^{2}$ \\ 1 CMAP, CNRS, Ecole Polytechnique, Institut Polytechnique de Paris, 91128 Palaiseau Cedex, France \\ 2 Laboratoire Interdisciplinaire Carnot de Bourgogne, CNRS, \\ Université Bourgogne Franche-Comté, Dijon, France and \\ ${ }^{3}$ Université Côte d'Azur, CNRS, Institut de Physique de Nice, Nice, France
}

\begin{abstract}
Classical nonlinear waves exhibit a phenomenon of condensation that results from the natural irreversible process of thermalization toward the Rayleigh-Jeans equilibrium spectrum. Wave condensation originates in the divergence of the thermodynamic equilibrium Rayleigh-Jeans distribution, which is responsible for the macroscopic population of the fundamental mode of the system. Several recent experiments revealed a remarkable phenomenon of spatial organization of an optical beam that propagates through a graded-index multimode optical fiber (MMF), a phenomenon termed beam self-cleaning. Our aim in this article is to provide some physical insight into the mechanism underlying optical beam self-cleaning through the analysis of wave condensation in the presence of structural disorder inherent to MMFs. We consider experiments of beam self-cleaning where long pulses are injected and populate many modes of a 10-20m MMF, for which the dominant contribution of disorder originates from polarization random fluctuations (weak disorder). On the basis of the wave turbulence theory, we derive nonequilibrium kinetic equations describing the random waves in a regime where disorder dominates nonlinear effects. The theory reveals that the presence of a conservative weak disorder introduces an effective dissipation in the system, which is shown to inhibit wave condensation in the usual continuous wave turbulence approach. On the other hand, the experiments of beam-cleaning are described by a discrete wave turbulence approach, where the effective dissipation induced by disorder modifies the regularization of wave resonances, which leads to an acceleration of condensation that can explain the effect of beam self-cleaning. By considering different models of weak disorder in MMFs, we show that a model where the modes experience a partially correlated noise is sufficient to accelerate the thermalization, whereas a fully mode-correlated noise does not lead to a dissipation-induced acceleration of condensation. The simulations are in quantitative agreement with the theory, and evidence an effect of beam-cleaning even in a regime of moderate weak disorder. At the leading order linear regime, random mode coupling among degenerate modes (strong disorder) can enforce thermalization and condensation. The analysis also reveals that the effect of beam cleaning is characterized by a partial repolarization as a natural consequence of the condensation process. In addition, the discrete wave turbulence approach explains why optical beam self-cleaning has not been observed in step-index multimode fibers.
\end{abstract}

\section{INTRODUCTION}

Bose-Einstein condensation refers to a quantum process characterized by a thermodynamic transition into a single, macroscopically populated coherent state. This phenomenon has been observed in a variety of quantum systems, such as ultracold atoms and molecules [1], exciton polaritons [2] and photons [3] (also see [4]). On the other hand, several studies on wave turbulence predicted that completely classical waves can undergo a condensation process [5-23]. The picture the reader may have in mind is the following. Considering an ensemble of weakly nonlinear dispersive random waves, a redistribution of energy occurs among different modes, which is responsible for a self-organization process: While the (kinetic) energy is transferred to the small scales fluctuations (higher modes), an inverse cascade increases the 'number of particles' into the lowest allowed mode, which leads to the emergence of a large scale coherent structure. This phenomenon refers to wave condensation. It originates in the natural thermalization of the wave system toward the thermodynamic Rayleigh-Jeans equilibrium distribution, whose divergence is responsible for the macroscopic occupation of the fundamental mode of the system $[6,7,10,13,14,17,20,24-27]$. We recall that this self-organization process takes place in a conservative (Hamiltonian) and formally reversible system: The ('condensate') remains immersed in a sea of small-scale fluctuations ('uncondensed particles'), which store the information for time reversal. In this respect, wave condensation is of different nature than other forms of condensation processes discussed in optical cavity systems, which are inherently nonequilibrium forced-dissipative systems $[20,28-36]$.

The observation of wave thermalization with optical waves in a (cavity-less) free propagation is known to require very large propagation lengths, as discussed recently in different circumstances $[23,26]$. The situation is different when the optical beam propagates in a waveguide configuration. The finite number of modes supported by the waveguide significantly reduces the rate of thermalization, in particular by regularizing the ultraviolet catastrophe inherent to classical waves [17]. In this framework, a remarkable phenomenon of spatial beam self-organization, termed 'beam self-cleaning', has been recently discovered in graded refractive index MMFs [3739]. At variance with an apparently similar effect driven by the dissipative Raman effect in MMFs, known as Ra- 
man beam cleanup [40], this self-organization is due to a purely conservative Kerr nonlinearity [39].

Recent works indicate that this phenomenon of beam self-cleaning can be interpreted as a consequence of wave thermalization and condensation [41-43]. In particular, in Ref.[41] a previously unrecognized mechanism of acceleration of condensation mediated by disorder has been reported, which can help to understand the effect of beam self-cleaning. Indeed, light propagation in MMFs is known to be affected by a structural disorder of the material due to inherent imperfections and external perturbations [44, 45], a feature which is attracting a growing interest, e.g., in image formation [46, 47], or to study the dynamics of completely integrable Manakov equations [48-53].

Our aim in this article is to provide some deeper physical insight into the mechanism underlying optical beam self-cleaning through the analysis of wave condensation in the presence of structural disorder of MMFs. We pursue the work initiated in Ref.[41] along different lines: (i) On the basis of the wave turbulence theory [6, 7, 20, 24] and related developments on finite size effects [55-66], we derive kinetic equations describing the nonequilibrium evolution of random waves in the regime where disorder effects dominate nonlinear effects. Considering the dominant contribution of polarization random fluctuations (weak disorder) [45], the theory shows that a conservative disorder introduces an effective dissipation in the evolution of the moments equations. By following the conventional continuous wave turbulence approach describing very highly MMFs, the analysis reveals that the effective dissipation introduces a frequency broadening of four wave resonances, which inhibits the conservation of the (kinetic) energy and, consequently, the effect of condensation. On the other hand, usual experiments of beam self-cleaning in MMFs are described by a discrete wave turbulence approach whereby dissipation induced by weak disorder modifies the regularization of wave resonances, which leads to a significant acceleration of the process of wave condensation. (ii) By considering different models of weak disorder in MMFs, the theory shows that when all modes experience the same (correlated) noise, the effective dissipation induced by disorder vanishes and the system no longer exhibits a fast process of condensation. On the other hand, even a small decorrelation among the noise experienced by the modes is sufficient to re-establish a dissipation-induced acceleration of condensation mediated by disorder. (iii) To improve our understanding of beam cleaning experiments, we have considered a regime where disorder effects are of the same order as nonlinear effects. In this case the simulations reveal the existence of a mixed coherent-incoherent regime, which is still characterized by a relatively fast process of self-cleaning condensation that is consistent with the experiments. (iv) By studying polarization effects [54], we show that optical beam cleaning is responsible for an effective repolarization of the central part of the optical beam, a feature that can be explained by the macroscopic condensed population of the fundamental mode of the MMF. (v) The discrete wave turbulence approach also explains why optical beam self-cleaning has not been observed in step-index MMFs. This is due to a significant reduction of the number of resonances and their corresponding efficiencies, which leads to an effective freezing of the process of thermalization and condensation. The same argument also explains why condensation is not observed in the recent experiments of beam cleaning under specific injection conditions [67], a feature that will be discussed in relation with the impact of a perturbation on the dispersion relations.

It is important to note that in this work we address the effect of optical beam self-cleaning in the quasicontinuous wave regime, where long (sub-nanosecond) temporal pulses are injected in the MMF. Temporal dispersion effects such as modal group-velocity mismatch and chromatic dispersion can be neglected within the short MMF lengths considered in these experiments. Accordingly, in this work we do not consider experiments of beam self-cleaning with high-power femto-second pulses [68], whose underlying mechanism should be related to complex spatio-temporal effects involving multimode solitons [69-74].

\section{THEORETICAL MODEL}

\section{A. Modal NLS equation}

We consider the (2+1)D nonlinear Schrödinger (NLS) equation (or Gross-Pitaevskii equation), which is known to describe the transverse spatial evolution of an optical beam propagating along the $z$-axis of a waveguide modelled by a confining potential $V(\boldsymbol{r})$ (with $\boldsymbol{r}=(x, y)$ ) [75]. Considering long temporal pulses (in the subnanosecond range), we neglect temporal effects related to first- and second-order chromatic dispersion. The vector NLS equation accounting for the polarization degree of freedom can then be written

$$
i \partial_{z} \boldsymbol{\psi}=-\alpha \nabla^{2} \boldsymbol{\psi}+V(\boldsymbol{r}) \boldsymbol{\psi}-\gamma_{0} \mathcal{P}(\boldsymbol{\psi}),
$$

where $\boldsymbol{\psi}(\boldsymbol{r}, z)=\left(\psi_{x}, \psi_{y}\right)^{T}$ with $\psi_{x, y}(\boldsymbol{r}, z)$ the vector field in the linear polarization basis, the superscript ${ }^{T}$ denoting the transpose operation. The parameter $\alpha=$ $1 /\left(2 k_{0} n_{0}\right)$ is the diffraction coefficient where $k_{0}=2 \pi / \lambda$ with $\lambda$ the vacuum laser wavelength and $n_{0}$ the refractive index of the fiber core. The nonlinear term reads:

$$
\mathcal{P}(\boldsymbol{\psi})=\frac{1}{3} \boldsymbol{\psi}^{T} \boldsymbol{\psi} \boldsymbol{\psi}^{*}+\frac{2}{3} \boldsymbol{\psi}^{\dagger} \boldsymbol{\psi} \boldsymbol{\psi},
$$

where the nonlinear coefficient is $\gamma_{0}=k_{0} n_{2}$, with $n_{2}$ the nonlinear Kerr coefficient, and the superscripts $*, \dagger$ stand for the conjugate, and conjugate transpose operations $\left(n_{2}>0\right.$ for a focusing fiber nonlinearity in $\mathrm{m}^{2} / \mathrm{W}$, $|\boldsymbol{\psi}|^{2}$ in $\left.\mathrm{W} / \mathrm{m}^{2}\right)$.

We expand the random wave into the orthonormal basis $\left(\int u_{p}(\boldsymbol{r}) u_{m}^{*}(\boldsymbol{r}) d \boldsymbol{r}=\delta_{p m}^{K}\right)$ of the eigenmodes $\left\{u_{p}(\boldsymbol{r})\right\}$ 
of the linearized NLS equation with the potential $V(\boldsymbol{r})$

$$
\boldsymbol{\psi}(\boldsymbol{r}, z)=\sum_{p=0}^{N_{*}-1} \boldsymbol{B}_{p}(z) u_{p}(\boldsymbol{r}) \exp \left(-i \beta_{p} z\right),
$$

where $N_{*}$ denotes the number of modes of the waveguide, $\boldsymbol{B}_{p}(z)=\left(B_{p, x}, B_{p, y}\right)^{T}$ refer to the linear polarization components of the $p$-th mode and $\beta_{p}$ are the corresponding eigenvalues verifying $\beta_{p} u_{p}(\boldsymbol{r})=-\alpha \nabla^{2} u_{p}(\boldsymbol{r})+$ $V(\boldsymbol{r}) u_{p}(\boldsymbol{r})$.

In the following we focus the analysis on GRIN (graded-index) MMFs in which the effect of beam self-cleaning has been observed experimentally, while the case of step-index MMFs will be discussed in Sec. VI A. For the ideal parabolic potential $V(\boldsymbol{r})=q|\boldsymbol{r}|^{2}$ for $|\boldsymbol{r}| \leq a$, $a$ being the fiber core radius, $u_{p}(\boldsymbol{r})$ refer to the normalized Hermite-Gaussian functions with corresponding eigenvalues $\beta_{p}=\beta_{p_{x}, p_{y}}=\beta_{0}\left(p_{x}+p_{y}+1\right), u_{p_{x}, p_{y}}(x, y)=$ $\kappa\left(\pi p_{x} ! p_{y} ! 2^{p_{x}+p_{y}}\right)^{-1 / 2} H_{p_{x}}(\kappa x) H_{p_{y}}(\kappa y) \exp \left[-\kappa^{2}\left(x^{2}+\right.\right.$ $\left.y^{2}\right) / 2$ ], where $\kappa=(q / \alpha)^{1 / 4}, q=k_{0}\left(n_{0}^{2}-n_{1}^{2}\right) /\left(2 n_{0} a^{2}\right)$, $\beta_{0}=2 \sqrt{\alpha q}, n_{1}$ being the cladding refractive index. The number of modes (without including polarization degeneracy) is $N_{*} \simeq V_{0}^{2} /\left(2 \beta_{0}^{2}\right)$, where $V_{0}$ denotes the depth of the parabolic potential. Defining $\boldsymbol{A}_{p}(z)=\boldsymbol{B}_{p}(z) \exp \left(-i \beta_{p} z\right)$, the evolutions of the modal components are governed by

$$
i \partial_{z} \boldsymbol{A}_{p}=\beta_{p} \boldsymbol{A}_{p}-\gamma \boldsymbol{P}_{p}(\boldsymbol{A}) .
$$

The nonlinear term in (4) reads

$$
\boldsymbol{P}_{p}(\boldsymbol{A})=\sum_{l, m, n=0}^{N_{*}-1} S_{p l m n}\left(\frac{1}{3} \boldsymbol{A}_{l}^{T} \boldsymbol{A}_{m} \boldsymbol{A}_{n}^{*}+\frac{2}{3} \boldsymbol{A}_{n}^{\dagger} \boldsymbol{A}_{m} \boldsymbol{A}_{l}\right),
$$

where $S_{l m n p}=\int u_{l}(\boldsymbol{r}) u_{m}(\boldsymbol{r}) u_{n}^{*}(\boldsymbol{r}) u_{p}^{*}(\boldsymbol{r}) d \boldsymbol{r} / \int\left|u_{0}\right|^{4}(\boldsymbol{r}) d \boldsymbol{r}$ so that $S_{0000}=1$, and $\gamma=\gamma_{0} / A_{\text {eff }}^{0}$, where $A_{\text {eff }}^{0}=$ $1 / \int\left|u_{0}\right|^{4}(\boldsymbol{r}) d \boldsymbol{r}$ is the effective area of the fundamental mode $\left(\left|\boldsymbol{A}_{p}\right|^{2}\right.$ in Watts).

The modal NLS Eq.(4) has a form analogous to the basic equation usually considered to study light propagation in MMFs in the absence of disorder [51, 52, 75-77]. It conserves the total power $N=\int|\boldsymbol{\psi}|^{2} d \boldsymbol{r}=\sum_{p=0}^{N_{*}-1}\left|\boldsymbol{A}_{p}\right|^{2}$, and the Hamiltonian $H=E+U$, which has a linear contribution $E=\alpha \int|\nabla \boldsymbol{\psi}|^{2} d \boldsymbol{r}+\int V(\boldsymbol{r})|\boldsymbol{\psi}|^{2} d \boldsymbol{r}=$ $\sum_{p=0}^{N_{*}-1} \beta_{p}\left|\boldsymbol{A}_{p}\right|^{2}$, and a nonlinear contribution:

$$
U=\frac{\gamma}{4} \sum_{l, m, n, p=0}^{N_{*}-1} S_{l m n p} \bar{U}_{l m n p}+\text { c.c. }
$$

where $\bar{U}_{l m n p}=\frac{1}{3}\left(\boldsymbol{A}_{l}^{T} \boldsymbol{A}_{m}\right)\left(\boldsymbol{A}_{n}^{\dagger} \boldsymbol{A}_{p}^{*}\right)+\frac{2}{3}\left(\boldsymbol{A}_{n}^{\dagger} \boldsymbol{A}_{m}\right)\left(\boldsymbol{A}_{l}^{T} \boldsymbol{A}_{p}^{*}\right)$ and c.c. denotes the complex conjugate.

The characteristic length $L_{0}=1 /(\gamma N)=$ $1 /\left(\gamma_{0} N / A_{\text {eff }}^{0}\right)$ corresponds to the nonlinear propagation length when all the power is confined in the fundamental mode. Usually the optical beam populates many higher-order modes and the nonlinear length is $L_{n l}=$
$1 /\left(\gamma_{0} P / S_{\text {eff }}\right)$ where $S_{\text {eff }}$ is the effective surface section of the beam. We also define the linear characteristic length from Eq.(4) as $L_{\text {lin }}=1 / \delta \beta_{\text {eff }}$, where $\delta \beta_{\text {eff }}$ denotes the effective ('spectral') bandwidth of the optical beam in the mode space. In usual experiments of beam self-cleaning in GRIN fibers we have $\beta_{0} \sim 10^{3} \mathrm{~m}^{-1}$, so that the optical wave evolves in the weakly nonlinear regime

$$
L_{l i n} \ll L_{n l},
$$

where $L_{\text {lin }} \lesssim 1 \mathrm{~mm}$ while $L_{n l}$ is typically larger than $10 \mathrm{~cm}$.

\section{B. Coherent modal regime: Impact of a confining parabolic potential on wave resonances}

In a typical experiment of optical beam self-cleaning a laser beam featured by a coherent transverse phase front is launched into the MMF. Under these conditions, the modal components excited at the fiber input exhibit a strong phase-correlation among each other. We remark that this is in contrast to usual simulations in wave turbulence where one imposes a random phase among the modes in the initial condition $[7,20,22,24,25]$. The numerical simulations of the NLS Eq.(4) reveal a remarkable fact: The strong phase-correlation among the low-order modes is preserved during the propagation, thus leading to a phase-sensitive mode interaction, as illustrated in Fig. 1(a). In this coherent regime, the phase relation-ship among the modes plays an important role in the dynamics. The low-order modal components $\boldsymbol{A}_{p}(z)$ that are strongly populated then experience a quasi-reversible exchange of power with each other. The corresponding intensity pattern $|\boldsymbol{\psi}|^{2}(\boldsymbol{r}, z)$ exhibits itself an oscillatory dynamics during the propagation: The beam populates several modes of the potential $V(\boldsymbol{r})$ and does not exhibit an enhanced brightness characterizing a stable self-cleaning effect. As already discussed in Ref.[41], this coherent regime of mode interactions freezes the thermalization process. In addition, we report in Fig. 1(b) a simulation of the NLS Eq.(4) starting from a speckle beam. Although the initial random phases modify the regular oscillatory behavior shown in Fig. 1(a), the low-order modal amplitudes still exhibit a rapid and significant power exchange among each other. This indicates that the phase relation-ship among the modes still plays a non-trivial role thus leading to an effective freezing of the process of thermalization. As will be discussed throughout this paper, these simulations reflect the discrete nature of the resonance manifold underlying wave turbulence in MMFs. We note that understanding the mechanisms that can freeze the process of thermalization is an important problem that is currently analyzed in various systems, such as, e.g., finite size effects in discrete or mesoscopic wave turbulence [55-66], in Fermi-PastaUlam chains [78-82], or in nonlinear disordered systems $[83,84]$. 

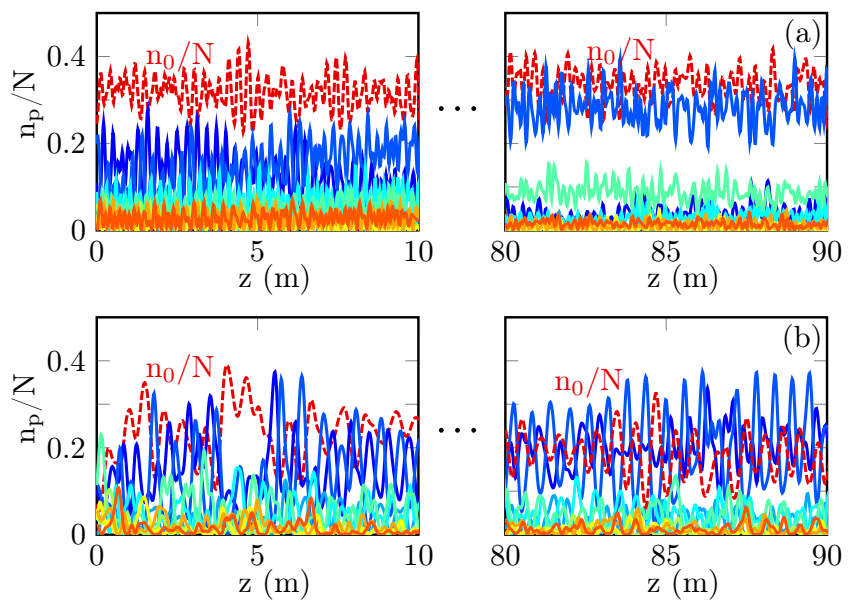

FIG. 1: Coherent regime of mode interaction:

Numerical simulations of the NLS Eq.(4) showing the evolutions of the modal components $n_{p}(z)=\left|\boldsymbol{A}_{p}(z)\right|^{2}$ (in the absence of disorder), starting from a coherent initial condition (a), starting from a 'speckle' beam with random phase among the modes (b). Evolutions of the modal populations $n_{p} / N$ : fundamental mode $p=0$ (red dashed), $p=1$ (dark blue solid), $p=2$ (blue solid), $p=3$ (light blue solid), $p=4$ (cyan solid), $p=5$ (light green solid), $p=6$ (green solid), $p=7$ (yellow solid), $p=8$ (orange solid). The system exhibits a persistent oscillatory dynamics among the low-order modes. At complete thermal equilibrium the system would reach the condensate fraction $n_{0}^{e q} / N \simeq 0.68$ in (a), and $n_{0}^{e q} / N \simeq 0.58$ in (b). The power is $N=47.5 \mathrm{~kW}$, $N_{*}=120$ modes, $a=26 \mu \mathrm{m}$.

The phase-sensitive regime of interaction among the highly populated modes of the MMF originates in the small number of modes confined in the parabolic potential (typically $\sim 15$ groups of degenerate modes, i.e., $\sim 120$ modes), which significantly reduces the effective number of modes that can interact efficiently. To illustrate this aspect, one can compare the number of resonances in a GRIN MMF used in the experiments of beam cleaning and the corresponding number of resonances in a 2D box. Let consider such two systems with approximately the same number of modes, say $N_{*}=120$ for the GRIN fiber and $N_{*}=121$ for the $2 \mathrm{D}$ box. In a $2 \mathrm{D}$ box with periodic boundary conditions the resonances verify energy conservation $\left(\omega_{\boldsymbol{k}_{1}}+\omega_{\boldsymbol{k}_{2}}=\omega_{\boldsymbol{k}_{3}}+\omega_{\boldsymbol{k}_{4}}, \omega_{\boldsymbol{k}}=\alpha|\boldsymbol{k}|^{2}\right)$, and momentum conservation $\left(\boldsymbol{k}_{1}+\boldsymbol{k}_{2}=\boldsymbol{k}_{3}+\boldsymbol{k}_{4}\right)$. In a GRIN fiber, the resonances verify energy conservation $\beta_{p}+\beta_{l}=\beta_{m}+\beta_{n}$, while the tensor $S_{l m n p}$ reflects the efficiency of the corresponding resonance. Because the eigenvalues $\beta_{p}$ are regularly spaced for a parabolic potential, there are much more resonances in a GRIN fiber than in a $2 \mathrm{D}$ box. However, most of the resonances are not efficient: only 41 (1067) resonances are characterized by a coefficient $S_{l m n p}>0.4\left(S_{l m n p}>0.2\right)$, where we re- call that $0<S_{l m n p} \leq 1$. In contrast, the $2 \mathrm{D}$ box exhibits $\sim 10^{5}$ exact resonances for energy and momentum conservation (the computation being independent on the box size). The analysis indicates that, as compared to the $2 \mathrm{D}$ box, the GRIN fiber effectively behaves as a dynamical system with a relative small number of degrees of freedom, which provides an intuitive interpretation for the persistent coherent regime of mode interaction discussed above. In the following we will see that the introduction of a structural disorder of the MMF breaks the coherent phase-sensitive regime of modal interaction.

\section{WEAK DISORDER: KINETIC EQUATIONS AND SIMULATIONS}

The modal NLS Eq.(4) assumes that the MMF is ideal, in the sense that the model does not account for any form of disorder. However, in practice light propagation in MMFs is known to be affected by random fluctuations of the longitudinal and transverse profiles of the index of refraction as a consequence of external factors such as bending, twisting, tensions, kinks, or core-size variations in the process of fabrication of the fiber. Such multiple physical perturbations introduce random polarization fluctuations as well as a random coupling among the modes of the fiber. The specific mechanisms and models that describe how fiber imperfections impact light propagation in MMFs still remains an active topic of research [44, 45, 49-53]. Usually, one considers that for relative short propagation lengths $(\sim 10 \mathrm{~m})$ as those considered in optical beam self-cleaning experiments, the dominant contribution of noise arises from polarization modal fluctuations (weak disorder), while for larger propagation lengths mode coupling occurs among degenerate modes (mode group coupling state of strong disorder), and for even larger fiber lengths mode coupling takes place for non-degenerate modes (strong disorder) [45]. In the following we consider the dominant contribution of weak disorder [45], while strong disorder will be considered in Sec. IV. We will see that the source of noise introduced by weak disorder is sufficient to break the coherent regime of modal interaction discussed here above in Sec. II B. The resulting random phase dynamics then leads to a turbulent incoherent regime of the modal components that we describe in the framework of the wave turbulence theory.

In the following we discuss in detail the impact of a weak disorder on the derivation of the wave turbulence kinetic equation for MMFs. We first discuss the conventional continuous wave turbulence regime relevant for very highly multimode fibers, and then the discrete wave turbulence regime relevant to the experiments of beam self-cleaning. We provide a detailed derivation of the discrete wave turbulence kinetic equation accounting for weak disorder - a sketch of the derivation was reported in Ref.[41]. We first consider a model of weak disorder in which the modes experience an independent 
(decorrelated) noise. We note that, in the opposite limit where the modes experience the same disorder, the analysis reveals that the optical wave recovers a quasi-coherent regime of mode interaction. In other words, this fully mode-correlated model of disorder appears ineffective and in this limit the optical wave does not exhibit a fast process of condensation. Accordingly, we consider an intermediate model of partially correlated disorder where degenerate modes experience the same noise. The theory shows that this partially mode-correlated disorder is sufficient to re-establish an efficient acceleration of condensation.

\section{A. Decorrelated model of disorder}

\section{Model}

As discussed above, in the weak disorder regime different spatial modes do not couple, but the two polarization states of each spatial mode exhibit a random coupling $[51,52]$. The evolutions of the modal components are governed by

$$
i \partial_{z} \boldsymbol{A}_{p}=\beta_{p} \boldsymbol{A}_{p}+\mathbf{D}_{p}(z) \boldsymbol{A}_{p}-\gamma \boldsymbol{P}_{p}(\boldsymbol{A}) .
$$

Let us recall that longitudinal and transverse fluctuations in the refractive index profile of the MMF lead to a random coupling among the modes, as expressed by the coupled-mode theory $[44,51]$. However, by following this procedure one obtains a scalar perturbation that does not introduce coupling among the polarization components, a feature commented in particular in Ref.[52]. Accordingly, random polarization fluctuations are usually introduced in a phenomenological way, see e.g. $[48,51,52]$. Here, we consider the most general form of polarization disorder that conserves the power of the optical beam. The Hermitian matrices $\mathbf{D}_{p}(z)$ are expanded into the Pauli matrices, which are known to form a basis for the vector space of $2 \times 2$ Hermitian matrices. The matrices then have the form

$$
\mathbf{D}_{p}(z)=\sum_{j=0}^{3} \nu_{p, j}(z) \boldsymbol{\sigma}_{j},
$$

where $\boldsymbol{\sigma}_{j}(j=1,2,3)$ are the Pauli matrices and $\boldsymbol{\sigma}_{0}$ is the identity matrix. The functions $\nu_{p, j}(z)$ are independent and identically distributed Gaussian real-valued random processes, with

$$
\left\langle\nu_{p, j}(z) \nu_{p^{\prime}, j^{\prime}}\left(z^{\prime}\right)\right\rangle=\sigma_{\beta}^{2} \delta_{p p^{\prime}}^{K} \delta_{j j^{\prime}}^{K} \mathcal{R}\left(\frac{z-z^{\prime}}{l_{\beta}}\right) .
$$

Here $l_{\beta}$ is the correlation length of the random process and $\sigma_{\beta}^{2}$ is its variance. The normalized correlation function is such that $\mathcal{R}(0)=O(1), \int_{-\infty}^{\infty} \mathcal{R}(\zeta) d \zeta=O(1)$, and $\mathcal{R}\left(z / l_{\beta}\right) \rightarrow l_{\beta} \delta(z)$ in the limit $l_{\beta} \rightarrow 0$. We will consider Ornstein-Uhlenbeck processes:

$$
d \nu_{p, j}=-\frac{1}{l_{\beta}} \nu_{p, j} d z+\frac{\sigma_{\beta}}{\sqrt{l_{\beta}}} d W_{p, j}(z)
$$

where $W_{p, j}$ are independent Brownian motions. This means that $\nu_{p, j}$ are Gaussian processes with mean zero and covariance function of the form (10) with $\mathcal{R}(\zeta)=$ $\exp (-|\zeta|) / 2$. We introduce an effective parameter of disorder $\Delta \beta=\sigma_{\beta}^{2} l_{\beta}$ and the associated length scale

$$
L_{d}=1 / \Delta \beta
$$

We assume that disorder effects dominate nonlinear effects

$$
L_{d} \ll L_{n l},
$$

and that $l_{\beta} \ll L_{d}$ (or $\sigma_{\beta} l_{\beta} \ll 1$ ). We recall that usual experiments of beam self-cleaning refer to a weakly nonlinear regime $L_{\text {lin }} \ll L_{n l}$, where we have typically $\beta_{0} \sim 10^{3} \mathrm{~m}^{-1}$, so that $\beta_{0}^{-1} \ll L_{d}$ (or $\Delta \beta \ll \beta_{0}$ ). Aside from beam-cleaning experiments, in the following we will also consider a regime where the mode-spacing can be very small $\beta_{0} \ll \Delta \beta$ (MMFs with huge number of modes $\left.N_{*} \simeq V_{0}^{2} /\left(2 \beta_{0}^{2}\right) \gg 1\right)$ so as to address a regime described by a continuous wave turbulence approach. Finally note that since the disorder is ('time') $z$-dependent, our system is of different nature than those studying the interplay of thermalization and Anderson localization [85].

\section{Vanishing correlations among modes}

First of all, we study the correlations among the modes through the analysis of the evolution of the second-order moments in the $2 \times 2$ matrix $\left\langle\boldsymbol{A}_{p}^{*} \boldsymbol{A}_{q}^{T}\right\rangle(z)$. The impact of disorder is treated by making use of the Furutsu-Novikov theorem. This reveals that the conservative disorder introduces an effective dissipation in the system. In this respect, we recall that in principle the laser beam excites strongly correlated modes at the fiber input, as discussed above through the coherent modal regime of interaction. It is the effective dissipation due to disorder that breaks such a strong modal phase-correlation.

The analysis developed in Appendix A (section IX A) completes that reported in the Supplemental of Ref.[41], which was focused on correlations between different modes, $\left\langle\boldsymbol{A}_{p}^{*} \boldsymbol{A}_{q}^{T}\right\rangle$ for $p \neq q$. The correlations within a single mode (i.e., among the orthogonal polarization components for $p=q$ ) is more delicate and it is reported in detail in Appendix A (section IX A). The theory reveals that in the regime discussed in Sec. III A 1, the correlations among different modes $(p \neq q)$, or within a single mode $(p=q)$, both have a form $\sim \gamma \boldsymbol{G}_{p q}(\boldsymbol{A}(z)) /(4 \Delta \beta-$ $\left.i\left(\beta_{p}-\beta_{q}\right)\right)$, where $\boldsymbol{G}_{p q}(\boldsymbol{A}(z))=\boldsymbol{P}_{p}(\boldsymbol{A}(z))^{*} \boldsymbol{A}_{q}^{T}(z)-$ $\boldsymbol{A}_{p}^{*}(z) \boldsymbol{P}_{q}(\boldsymbol{A}(z))^{T}$. Recalling that $L_{d} \ll L_{n l}$ we see that, at leading order, modal correlations vanish for propagation lengths larger than the nonlinear length, $\left\langle\boldsymbol{A}_{p}^{*} \boldsymbol{A}_{q}^{T}\right\rangle(z) \simeq 0$ for $z \gg L_{n l}$. 


\section{Closure of the moments equations}

In the following we derive the kinetic equation by following the wave turbulence perturbation expansion procedure in which linear dispersive effects dominate nonlinear effects $L_{l i n} \ll L_{n l}$. Accordingly, an effective large separation of the linear and the nonlinear lengths scales takes place $[6,7]$. When combined with disorder effects, the modes exhibit random phases with quasi-Gaussian statistics, which allows one to achieve the closure of the infinite hierarchy of moment equations. More precisely, because of the nonlinear character of the NLS equation, the evolution of the second-order moment of the field depends on the fourth-order moment, while the equation for the fourth-order moment depends on the sixth-order moment, and so on. In this way, one obtains an infinite hierarchy of moment equations, in which the $n$-th order moment depends on the $(n+2)$-th order moment of the field. This makes the equations impossible to solve unless some way can be found to truncate the hierarchy. The closure of the infinite hierarchy of moment equations can be realized in the weakly nonlinear regime by virtue of the Gaussian moment theorem. We remark in this respect that the key assumption underlying the wave turbulence approach is the existence of a random phase among the modes rather than a genuine Gaussian statistics, as recently discussed in detail in Refs. $[7,86,87]$. We emphasize that in the present work the random phase of the modes is induced by the structural disorder of the medium that dominates nonlinear effects $\left(L_{d} \ll L_{n l}\right)$.

As discussed here above, the nondiagonal components of the $2 \times 2$ matrix $\left\langle\boldsymbol{A}_{p}^{*} \boldsymbol{A}_{p}^{T}\right\rangle(z)$ vanish. Then our aim is to derive an equation governing the evolutions of the diagonal components $w_{p}(z)=\frac{1}{2}\left\langle\left|\boldsymbol{A}_{p}(z)\right|^{2}\right\rangle$. Starting from the modal NLS Eq.(8), we have

$$
\begin{aligned}
\partial_{z} w_{p} & =\frac{1}{3} \gamma\left\langle X_{p}^{(1)}\right\rangle+\frac{2}{3} \gamma\left\langle X_{p}^{(2)}\right\rangle, \\
X_{p}^{(1)} & =\operatorname{Im}\left\{\sum_{l, m, n} S_{l m n p}^{*}\left(\boldsymbol{A}_{l}^{\dagger} \boldsymbol{A}_{m}^{*}\right)\left(\boldsymbol{A}_{n}^{T} \boldsymbol{A}_{p}\right)\right\}, \\
X_{p}^{(2)} & =\operatorname{Im}\left\{\sum_{l, m, n} S_{l m n p}^{*}\left(\boldsymbol{A}_{n}^{T} \boldsymbol{A}_{m}^{*}\right)\left(\boldsymbol{A}_{l}^{\dagger} \boldsymbol{A}_{p}\right)\right\} .
\end{aligned}
$$

The detailed derivation of the equations for the fourth order correlators $J_{l m n p}^{(1)}(z)=\left\langle\left(\boldsymbol{A}_{l}^{\dagger} \boldsymbol{A}_{m}^{*}\right)\left(\boldsymbol{A}_{n}^{T} \boldsymbol{A}_{p}\right)\right\rangle$ and $J_{l m n p}^{(2)}(z)=\left\langle\left(\boldsymbol{A}_{n}^{T} \boldsymbol{A}_{m}^{*}\right)\left(\boldsymbol{A}_{l}^{\dagger} \boldsymbol{A}_{p}\right)\right\rangle$ is given in the Appendix A (section IX B). As already noticed, as a result of the Furutsu-Novikov theorem, the conservative disorder introduces an effective dissipation in the system, so that the evolutions of the fourth-order moments have the form of a forced-damped oscillator equation:

$$
\left.\partial_{z} J_{l m n p}^{(j)}=\left(-8 \Delta \beta+i \Delta \omega_{l m n p}\right) J_{l m n p}^{(j)}+i \gamma\left\langle Y_{l m n p}^{(j)}\right\rangle 16\right)
$$

where $\Delta \omega_{l m n p}=\beta_{l}+\beta_{m}-\beta_{n}-\beta_{p}$ is the frequency resonance, while $\left\langle Y_{l m n p}^{(j)}\right\rangle$ denote the six-th order moments that have been split into products of second-order moments by virtue of the factorizability property of statistical Gaussian fields (see the detailed expressions of $\left\langle Y_{l m n p}^{(j)}\right\rangle$ for $j=1,2$ in Eq.(52) and Eq.(59) in the Appendix A, section IX B). The solution to Eq.(16) reads

$$
J_{l m n p}^{(j)}(z)=J_{l m n p}^{(j)}(0) G_{l m n p}(z)+\mathcal{I}_{l m n p}^{(j)}(z)
$$

where the convolution integral reads

$$
\mathcal{I}_{l m n p}^{(j)}(z)=i \gamma \int_{0}^{z}\left\langle Y_{l m n p}^{(j)}\right\rangle\left(z-z^{\prime}\right) G_{l m n p}\left(z^{\prime}\right) d z^{\prime}
$$

with the Green function

$$
G_{l m n p}(z)=H(z) \exp \left(i \Delta \omega_{l m n p} z-8 \Delta \beta z\right),
$$

and $H(z)$ the Heaviside function. We now arrive at the key point of our analysis, in which one distinguishes the continuous from the discrete wave turbulence approaches.

\section{Continuous wave turbulence}

In general, an exchange of power among four different modes does not need to satisfy the exact resonance condition $\Delta \omega_{l m n p}=0$. Indeed, it is sufficient for a mode quartet to verify the quasi-resonant condition

$$
\left|\Delta \omega_{l m n p}\right| \lesssim 1 / L_{k i n}^{\text {disor }}
$$

to provide a non-vanishing contribution to the convolution integral (18), where $L_{k i n}^{\text {disor }}$ denotes the characteristic evolution length scale of the moments of the field $\left\langle Y_{l m n p}^{(j)}\right\rangle(z)$ (see Eq.(26) below). In the usual continuous wave turbulence approach, there are a large number of non-resonant mode quartets $\Delta \omega_{l m n p} \neq 0$ that verify the quasi-resonant condition (20) and that contribute significantly to the evolution of the moments equations.

We anticipate that the continuous wave turbulence regime does not correspond to the usual experiments of optical beam self-cleaning [38, 39], since we have typically $\beta_{0} \sim 10^{3} \mathrm{~m}^{-1}$, so that $\beta_{0} \gg 1 / L_{\text {kin }}^{\text {disor }}$. In other words, since any non-resonant mode quartet verifies $\left|\Delta \omega_{l m n p}\right| \geq \beta_{0} \gg 1 / L_{\text {kin }}^{\text {disor }}$, the system does not exhibit quasi-resonances, but solely exact resonances $\Delta \omega_{l m n p}=$ 0 . Actually, the experiments of beam self-cleaning are described by the discrete wave turbulence approach that will be discussed in detail in the next Sec. III A 5. Here, for the sake of clarity, we first discuss the conventional continuous wave turbulence regime [7], which can be relevant for MMFs characterized by a huge number of modes $\left(N_{*} \simeq V_{0}^{2} /\left(2 \beta_{0}^{2}\right) \gg 1\right.$ such that $\left.\beta_{0} \ll \Delta \beta\right)$. The discussion of the usual continuous regime is also important in that it enlightens the effect of acceleration of thermalization due to the presence of structural disorder in the system.

Recalling that $L_{d}=1 / \Delta \beta \ll L_{n l}$, then the Green function decays on a length scale much smaller than the 
evolution length of $\left\langle Y_{l m n p}^{(j)}\right\rangle(z)$, so that the convolution integral can be approximated for $z \gg L_{d}$

$$
J_{l m n p}^{(j)} \simeq \gamma\left\langle Y_{l m n p}^{(j)}\right\rangle \frac{i 8 \Delta \beta-\Delta \omega_{l m n p}}{\Delta \omega_{l m n p}^{2}+(8 \Delta \beta)^{2}} .
$$

By substitution of Eq.(21) in the fourth-order moments (17), one obtains the expressions of the averaged moments $\left\langle X_{p}^{(j)}\right\rangle$ given in (14-15). Collecting all terms in (13) gives the equation for the modal amplitudes $n_{p}(z)=2 w_{p}(z)$. Since the MMF supports a large number of modes $N_{*} \simeq V_{0}^{2} /\left(2 \beta_{0}^{2}\right) \gg 1$, we consider the continuous limit where the discrete sums in (14-15) are replaced by continuous integrals $\left(\beta_{0} / V_{0} \ll 1\right)$. We obtain the continuous kinetic equation

$$
\begin{aligned}
& \partial_{z} \tilde{n}_{\boldsymbol{\kappa}_{4}}(z)=\frac{4 \gamma^{2}}{3 \beta_{0}^{6}} \iiint d \boldsymbol{\kappa}_{1,2,3} \frac{\overline{\Delta \beta}}{\Delta \tilde{\omega}_{\boldsymbol{\kappa}_{1} \boldsymbol{\kappa}_{2} \boldsymbol{\kappa}_{3} \boldsymbol{\kappa}_{4}}^{2}+\overline{\Delta \beta}^{2}} \\
& \times\left|\tilde{S}_{\kappa_{1} \kappa_{2} \kappa_{3} \kappa_{4}}\right|^{2} \tilde{M}_{\kappa_{1} \kappa_{2} \kappa_{3} \kappa_{4}} \\
& +\frac{32 \gamma^{2}}{9 \beta_{0}^{2}} \int d \boldsymbol{\kappa}_{1} \frac{\overline{\Delta \beta}}{\Delta \tilde{\omega}_{\boldsymbol{\kappa}_{1} \boldsymbol{\kappa}_{4}}^{2}+\overline{\Delta \beta}^{2}}\left|\tilde{s}_{\boldsymbol{\kappa}_{1} \boldsymbol{\kappa}_{4}}(\tilde{\boldsymbol{n}})\right|^{2}\left(\tilde{n}_{\boldsymbol{\kappa}_{1}}-\tilde{n}_{\boldsymbol{\kappa}_{4}}\right)
\end{aligned}
$$

where $d \boldsymbol{\kappa}_{1,2,3}=d \boldsymbol{\kappa}_{1} d \boldsymbol{\kappa}_{2} d \boldsymbol{\kappa}_{3}, \overline{\Delta \beta}=8 \Delta \beta$ and

$$
\tilde{s}_{\boldsymbol{\kappa}_{1} \boldsymbol{\kappa}_{4}}(\tilde{\boldsymbol{n}})=\frac{1}{\beta_{0}^{2}} \int d \boldsymbol{\kappa}^{\prime} \tilde{S}_{\boldsymbol{\kappa}_{1} \boldsymbol{\kappa}^{\prime} \boldsymbol{\kappa}^{\prime} \boldsymbol{\kappa}_{4}} \tilde{n}_{\boldsymbol{\kappa}^{\prime}}
$$

The functions with a tilde refer to the natural continuum extension of the corresponding discrete functions, i.e., $\tilde{n}_{\boldsymbol{k}}(z)=n_{\left[\boldsymbol{k} / \beta_{0}\right]}(z), \quad \tilde{\beta}_{\boldsymbol{\kappa}}=\beta_{\left[\boldsymbol{\kappa} / \beta_{0}\right]}, \quad \tilde{S}_{\boldsymbol{\kappa}_{1} \boldsymbol{\kappa}_{2} \kappa_{3} \kappa_{4}}=$ $S_{\left[\boldsymbol{\kappa}_{1} / \beta_{0}\right]\left[\boldsymbol{\kappa}_{2} / \beta_{0}\right]\left[\boldsymbol{\kappa}_{3} / \beta_{0}\right]\left[\boldsymbol{\kappa}_{4} / \beta_{0}\right]}$ and so on, where $[x]$ denotes the integer part of $x$. With these notations we have $\tilde{M}_{\boldsymbol{\kappa}_{1} \boldsymbol{\kappa}_{2} \boldsymbol{\kappa}_{3} \boldsymbol{\kappa}_{4}}=\tilde{n}_{\boldsymbol{\kappa}_{1}} \tilde{n}_{\boldsymbol{\kappa}_{2}} \tilde{n}_{\boldsymbol{\kappa}_{3}} \tilde{n}_{\boldsymbol{\kappa}_{4}}\left(\tilde{n}_{\boldsymbol{\kappa}_{4}}^{-1}+\tilde{n}_{\boldsymbol{\kappa}_{3}}^{-1}-\tilde{n}_{\boldsymbol{\kappa}_{1}}^{-1}-\tilde{n}_{\boldsymbol{\kappa}_{2}}^{-1}\right)$, $\Delta \tilde{\omega}_{\kappa_{1} \kappa_{2} \kappa_{3} \kappa_{4}}=\tilde{\beta}_{\kappa_{1}}+\tilde{\beta}_{\boldsymbol{\kappa}_{2}}-\tilde{\beta}_{\kappa_{3}}-\tilde{\beta}_{\boldsymbol{\kappa}_{4}}, \Delta \tilde{\omega}_{\kappa_{1} \kappa_{2}}=\tilde{\beta}_{\boldsymbol{\kappa}_{1}}-\tilde{\beta}_{\boldsymbol{\kappa}_{2}}$, and $\tilde{\beta}_{\boldsymbol{\kappa}}=\kappa_{x}+\kappa_{y}+\beta_{0}$, with $\boldsymbol{\kappa}=\beta_{0}\left(p_{x}, p_{y}\right)$ [17].

The main novelty of the kinetic Eq.(23) with respect to the previous work without any structural disorder [17], is that the mechanism of disorder-induced dissipation introduces a finite bandwidth into the four-wave resonances among the modes. Accordingly, instead of the Dirac $\delta$ distribution that guarantees energy conservation at each four-wave interaction, here the kinetic equation involves a Lorentzian distribution. We remark that this aspect was already discussed in different circumstances [5], in particular in the recent work [34] dealing with random fiber lasers in the presence of gain and losses. Here, the originality is that the finite bandwidth of the Lorentzian distribution and the associated effective dissipation $\Delta \beta$ originate in the conservative structural disorder of the material.

Taking the formal limit $\Delta \beta \rightarrow 0: \overline{\Delta \beta} /\left(\Delta \tilde{\omega}_{\kappa_{1} \kappa_{2} \kappa_{3} \kappa_{4}}^{2}+\right.$ $\left.\overline{\Delta \beta}^{2}\right) \rightarrow \pi \delta\left(\Delta \tilde{\omega}_{\kappa_{1} \kappa_{2} \kappa_{3} \kappa_{4}}\right)$, we recover a continuous kinetic equation with a form similar to that derived in [17] in the absence of disorder $(\Delta \beta=0)$ and in the absence of polarization effects (scalar limit $\boldsymbol{A}_{p} \rightarrow A_{p, x}$ ). However the above limit $\Delta \beta \rightarrow 0$ is not physically relevant here since we have assumed $L_{d}=1 / \Delta \beta \ll L_{n l}$ to derive Eq.(16). Here, we consider the regime $\beta_{0} \ll \Delta \beta$, so that it is the opposite limit that is physically relevant $\overline{\Delta \beta} /\left(\Delta \tilde{\omega}_{\kappa_{1} \kappa_{2} \kappa_{3} \kappa_{4}}^{2}+\overline{\Delta \beta}^{2}\right) \rightarrow 1 / \overline{\Delta \beta}$, and the kinetic equation takes the reduced form:

$$
\begin{array}{r}
\partial_{z} \tilde{n}_{\boldsymbol{\kappa}_{4}}(z)=\frac{\gamma^{2}}{6 \Delta \beta \beta_{0}^{6}} \iiint d \boldsymbol{\kappa}_{1,2,3}\left|\tilde{S}_{\boldsymbol{\kappa}_{1} \boldsymbol{\kappa}_{2} \boldsymbol{\kappa}_{3} \boldsymbol{\kappa}_{4}}\right|^{2} \tilde{M}_{\boldsymbol{\kappa}_{1} \boldsymbol{\kappa}_{2} \boldsymbol{\kappa}_{3} \boldsymbol{\kappa}_{4}} \\
+\frac{4 \gamma^{2}}{9 \Delta \beta \beta_{0}^{2}} \int d \boldsymbol{\kappa}_{1}\left|\tilde{s}_{\boldsymbol{\kappa}_{1} \boldsymbol{\kappa}_{4}}(\tilde{\boldsymbol{n}})\right|^{2}\left(\tilde{n}_{\boldsymbol{\kappa}_{1}}-\tilde{n}_{\boldsymbol{\kappa}_{4}}(24)\right.
\end{array}
$$

The second term in the right-hand side of (24) enforces the isotropization of the mode occupancies $\tilde{n}_{\kappa}(z)$ among the degenerate modes, while the first term enforces the mode occupancies to reach the most disordered equilibrium distribution. The kinetic equation (24) conserves the power, $N=\beta_{0}^{-2} \int \tilde{n}_{\boldsymbol{\kappa}} d \boldsymbol{\kappa}$, and exhibits a $H$-theorem of entropy growth, $\partial_{z} \mathcal{S}(z) \geq 0$, where the nonequilibrium entropy is defined by $\mathcal{S}(z)=\beta_{0}^{-2} \int \log \left(\tilde{n}_{\boldsymbol{\kappa}}\right) d \boldsymbol{\kappa}$. However, at variance with the conventional wave turbulence kinetic equation, the kinetic Eq.(24) does not conserve the energy, $E=\beta_{0}^{-2} \int \tilde{\beta}_{\boldsymbol{\kappa}} \tilde{n}_{\boldsymbol{\kappa}} d \boldsymbol{\kappa}$. Then the equilibrium distribution maximizing the entropy given the constraint on the conservation of the power $N$ is given by the uniform distribution

$$
\tilde{n}_{\kappa}^{e q}=\text { const. }
$$

This equilibrium state denotes an equipartition of ('particles') power among all modes. Recalling that $\beta_{0} \ll \Delta \beta$, the Lorentzian distribution in the kinetic Eq.(22) is dominated by disorder, so that the length scale characterizing the rate of thermalization toward the equilibrium distribution (25) is given by

$$
L_{\text {kin }}^{\text {disor }} \sim \Delta \beta L_{n l}^{2} / \bar{S}_{l m n p}^{2},
$$

where $\bar{S}_{\text {lmnp }}^{2}$ denotes the average square of the tensor $S_{l m n p}$ involving non-trivial resonances among nondegenerate modes.

The main conclusion is that the kinetic Eq.(23) does not describe a process of condensation characterized by a macroscopic occupation of the fundamental mode. This indicates that weak disorder should prevent an effect of beam self-cleaning in MMFs featured by huge number of modes.

\section{Discrete wave turbulence}

We have seen that in the continuous wave turbulence regime quasi-resonances verifying $\Delta \omega_{l m n p} \lesssim 1 / L_{\text {kin }}^{\text {disor }}$ contribute to the convolution integral (18). At variance with the continuous regime, in the discrete case the nonvanishing minimum value of $\Delta \omega_{l m n p}$ is such that

$$
\min \left(\left|\Delta \omega_{l m n p}\right|\right)=\beta_{0} \gg 1 / L_{\text {kin }}^{\text {disor }} .
$$

Accordingly, only exact resonances $\Delta \omega_{l m n p}=0$ contribute to the integral (18), while non-resonant mode 
quartets lead to a vanishing integral [7]. Note that this procedure can also be justified by a homogenization procedure, as reported in [65] in the presence of a nonconservative disorder accounting for gain and losses in the system. As discussed above, usual experiments of optical beam self-cleaning are described by the discrete wave turbulence approach since $\beta_{0}^{-1} \simeq 10^{-3} \mathrm{~m}$ and the above condition is well verified in the experiments [38, 39].

In the discrete wave turbulence regime we need to consider separately the cases of resonant and nonresonant mode interactions. For mode quartets verifying $\Delta \omega_{l m n p}=0$, the Green function (19) decays on a length scale much smaller than the evolution of $n_{p}(z)$, because $L_{d}=(\Delta \beta)^{-1} \ll L_{k i n}$, so that (18) can be approximated by $J_{l m n p}^{(j)}(z) \simeq \frac{i \gamma}{8 \Delta \beta}\left\langle Y_{l m n p}^{(j)}\right\rangle(z)$. For $\Delta \omega_{l m n p} \neq 0$, the Green function oscillates rapidly over a length scale smaller than $L_{l i n}=\beta_{0}^{-1} \ll L_{k i n}$. Such a rapid phase rotation combined to the fast decay of the Green function over a length $L_{d} \ll L_{n l}$ leads to a vanishing convolution integral in (18). As a result, the fourth-order moment can be written in the form

$$
J_{l m n p}^{(j)}(z) \simeq \frac{i \gamma}{8 \Delta \beta}\left\langle Y_{l m n p}^{(j)}\right\rangle(z) \delta^{K}\left(\Delta \omega_{l m n p}\right),
$$

where $\delta^{K}\left(\Delta \omega_{l m n p}\right)=1$ if $\Delta \omega_{l m n p}=0$, and zero otherwise. Note that the discrete regime discussed here does not exactly correspond to the discrete regimes due to finite size effects in homogeneous wave turbulence [7] here the system is non-homogeneous $(V(\boldsymbol{r}) \neq$ const $)$ and the resonance for the momentum reflected by the tensor $S_{l m n p}$ is not as rigid as the usual one involving the Dirac $\delta$ distribution in homogeneous turbulence.

We provide a detailed computation of the fourth-order moments $J_{l m n p}^{(j)}(z)$ and corresponding moments $\left\langle X_{p}^{(j)}\right\rangle$ defined in (14-15) in the Appendix A (section IX B), see Eq.(56) and (60). Then collecting all terms in (13) give the discrete kinetic equation for the modal amplitudes $n_{p}(z)=2 w_{p}(z)$

$$
\begin{aligned}
\partial_{z} n_{p}(z)= & \frac{\gamma^{2}}{6 \Delta \beta} \sum_{l, m, n}\left|S_{l m n p}\right|^{2} \delta^{K}\left(\Delta \omega_{l m n p}\right) M_{l m n p}(\boldsymbol{n}) \\
& +\frac{4 \gamma^{2}}{9 \Delta \beta} \sum_{l}\left|s_{l p}(\boldsymbol{n})\right|^{2} \delta^{K}\left(\Delta \omega_{l p}\right)\left(n_{l}-n_{p}\right),
\end{aligned}
$$

with $s_{l p}(\boldsymbol{n})=\sum_{m^{\prime}} S_{l m^{\prime} m^{\prime} p} n_{m^{\prime}}$, and $M_{l m n p}(\boldsymbol{n})=$ $n_{l} n_{m} n_{p}+n_{l} n_{m} n_{n}-n_{n} n_{p} n_{m}-n_{n} n_{p} n_{l}$, with ' $n_{m}$ ' for ' $n_{m}(z)$ ', $\Delta \omega_{l p}=\beta_{l}-\beta_{p}$. According to the kinetic equation (29), the length scale characterizing the rate of thermalization is the same as that obtained in the continuous wave turbulence regime (26), namely $L_{k i n}^{\text {disor }} \sim$ $\Delta \beta L_{n l}^{2} / \bar{S}_{l m n p}^{2}$.

Aside from its discrete form, the kinetic Eq.(29) has a structure analogous to the conventional wave turbulence kinetic equation, so that it describes a process of wave condensation that occurs irrespective of the sign of the nonlinearity $\gamma$ (see the factor $\gamma^{2}$ in Eq.(29)) [7, 10].
We refer the reader to Refs. $[7,10,13]$ for details on condensation in the homogeneous case $(V(\boldsymbol{r})=0)$, and to [17] for the non-homogeneous case in a waveguide potential $(V(\boldsymbol{r}) \neq 0)$. It is important to note that, in contrast to the kinetic Eq.(24) derived in the continuous wave turbulence regime, here Eq.(29) also conserves the energy $E=\sum_{p} \beta_{p} n_{p}(z)$ - despite the presence of the dissipation effect $\Delta \beta$. The reason for this is that only exact resonances contribute to the discrete turbulence regime, so that the discrete kinetic equation is not affected by the dissipation-induced resonance broadening that inhibits energy conservation in the continuous turbulence regime. The kinetic Eq.(29) also conserves the 'number of particles' $N=\sum_{p} n_{p}(z)$ and exhibits a $H$-theorem of entropy growth for the nonequilibrium entropy $\mathcal{S}(z)=\sum_{p} \log \left(n_{p}(z)\right)$. Accordingly, it describes an irreversible evolution to the (maximum entropy) thermodynamic Rayleigh-Jeans equilibrium

$$
n_{p}^{e q}=T /\left(\beta_{p}-\mu\right) .
$$

The system exhibits a phase transition to condensation when $\mu \rightarrow \beta_{0}$ [17]: for $E \geq E_{\text {crit }}=\frac{N V_{0}}{2}\left(1+2 \beta_{0} / V_{0}\right)$ there is no condensation $n_{0}^{e q} / N=0$, while for $E<E_{\text {crit }}$ the fundamental mode of the MMF gets macroscopically populated

$$
\frac{n_{0}^{e q}}{N}=1-\frac{E-E_{0}}{E_{\text {crit }}-E_{0}},
$$

where $E_{0}=N \beta_{0}$ is the minimum energy (all particles are in the fundamental mode). Note that Eq.(31) only provides an approximation of the condensation curve $n_{0}^{e q}$ vs $E$, see Ref.[17] for a more detailed discussion. As discussed in [41], a stable self-cleaned shape of the intensity pattern $|\psi|^{2}(\boldsymbol{r})$ can be interpreted as a consequence of the macroscopic population of the fundamental mode of the MMF (see the movie published in the Supplemental of [41]).

\section{Acceleration of thermalization mediated by disorder}

The length scale characterizing the rate of thermalization in the absence of structural disorder is obtained from the continuous kinetic equation that was derived in Ref.[17], $L_{k i n}^{\text {ord }} \sim \beta_{0} L_{n l}^{2} / \bar{S}_{l m n p}^{2}$. As discussed in Appendix $\mathrm{B}$, a similar scaling behavior is expected in the discrete wave turbulence regime. Hence, in usual regimes of beam self-cleaning experiments where $\beta_{0} \gg \Delta \beta$, the weak disorder is responsible for a significant acceleration of the rate of thermalization and condensation

$$
L_{k i n}^{\text {disor }} / L_{k i n}^{\text {ord }} \sim \Delta \beta / \beta_{0} .
$$

Considering typical values of $\beta_{0} \sim 10^{3} \mathrm{~m}^{-1}$ and $L_{d}=$ $1 / \Delta \beta$ larger than tens of centimetres, we see that we always have $L_{k i n}^{\text {disor }} / L_{k i n}^{\text {ord }} \ll 1$. Accordingly, weak disorder is responsible for a significant acceleration of the rate 


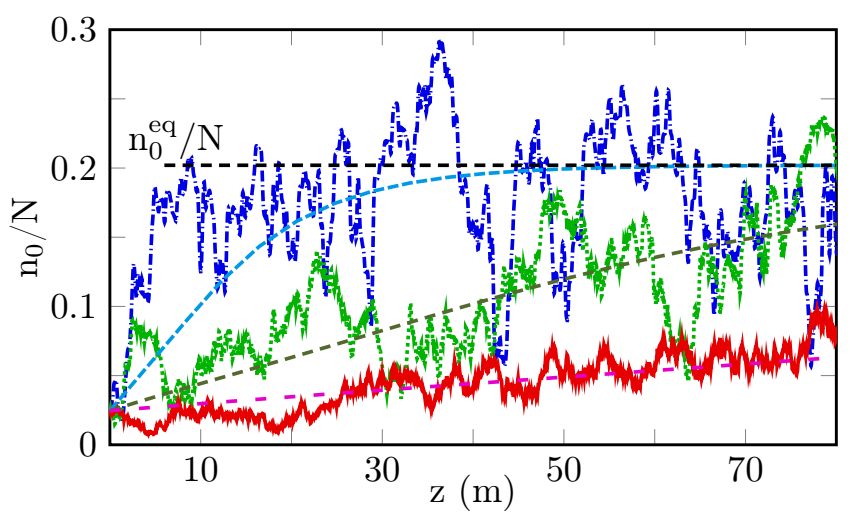

FIG. 2: Scaling of acceleration of thermalization with mode-decorrelated disorder: Numerical simulations of the NLS Eq.(8) showing the evolutions of the

fundamental mode $n_{0}(z)$, for different amounts of disorder $\Delta \beta$. The dashed lines show the corresponding simulations of the discrete kinetic Eq.(29), starting from

the same initial condition as the NLS simulations.

Parameters are: $\Delta \beta \simeq 2.6 \mathrm{~m}^{-1}\left(2 \pi / \sigma_{\beta}=2.1 \mathrm{~m}\right.$, $l_{\beta}=30 \mathrm{~cm}$ ) blue (dashdotted); $\Delta \beta \simeq 10.5 \mathrm{~m}^{-1}$, $\left(2 \pi / \sigma_{\beta}=26 \mathrm{~cm}, l_{\beta}=1.88 \mathrm{~cm}\right)$ green (dotted); $\Delta \beta \simeq 42 \mathrm{~m}^{-1},\left(2 \pi / \sigma_{\beta}=6.6 \mathrm{~cm}, l_{\beta}=0.47 \mathrm{~cm}\right)$ red (solid); while the power is $N=47.5 \mathrm{~kW}\left(N_{*}=120\right.$ modes, $a=26 \mu \mathrm{m})$. The curves eventually relax to the common theoretical equilibrium value $n_{0}^{e q} / N \simeq 0.2$ (dashed black line from Eq.(31)) with different rates, confirming the scaling of acceleration of condensation predicted by the theory in Eq.(32), without using adjustable parameters.

of thermalization and condensation [41]. Note that the presence of a perturbation on the dispersion relation can modify the above scaling, a feature that will be discussed later.

We have confirmed the scaling (32) by performing numerical simulations of the NLS Eq.(8) for different amounts of disorder $\Delta \beta$. We refer the reader to the Supplemental of Ref.[41] for the numerical scheme used to solve the NLS Eq.(8) in the presence of disorder. The results are reported in Fig. 2 and confirm the scaling of the rate of acceleration of thermalization and condensation predicted by the theory. More precisely, a remarkable quantitative agreement has been obtained between the simulations of the NLS Eq.(8) and those of the discrete kinetic Eq.(29), without using any adjustable parameter. Note that the equilibrium value of the condensate fraction $n_{0}^{e q} / N$ vs the energy $E$ is obtained from a rather simple analysis of the RJ equilibrium distribution $[7,10,17]$. In our case, the condensation curve $n_{0}^{e q} / N$ vs the energy $E$ has been reported in explicit form in Ref.[41] (see Eqs.(17-18) in the Supplemental). Also note that we have deliberately chosen a small value of the condensate fraction $n_{0} / N \simeq 0.2$ so as to avoid large deviations from Gaussianity for the fundamental mode - though the the-
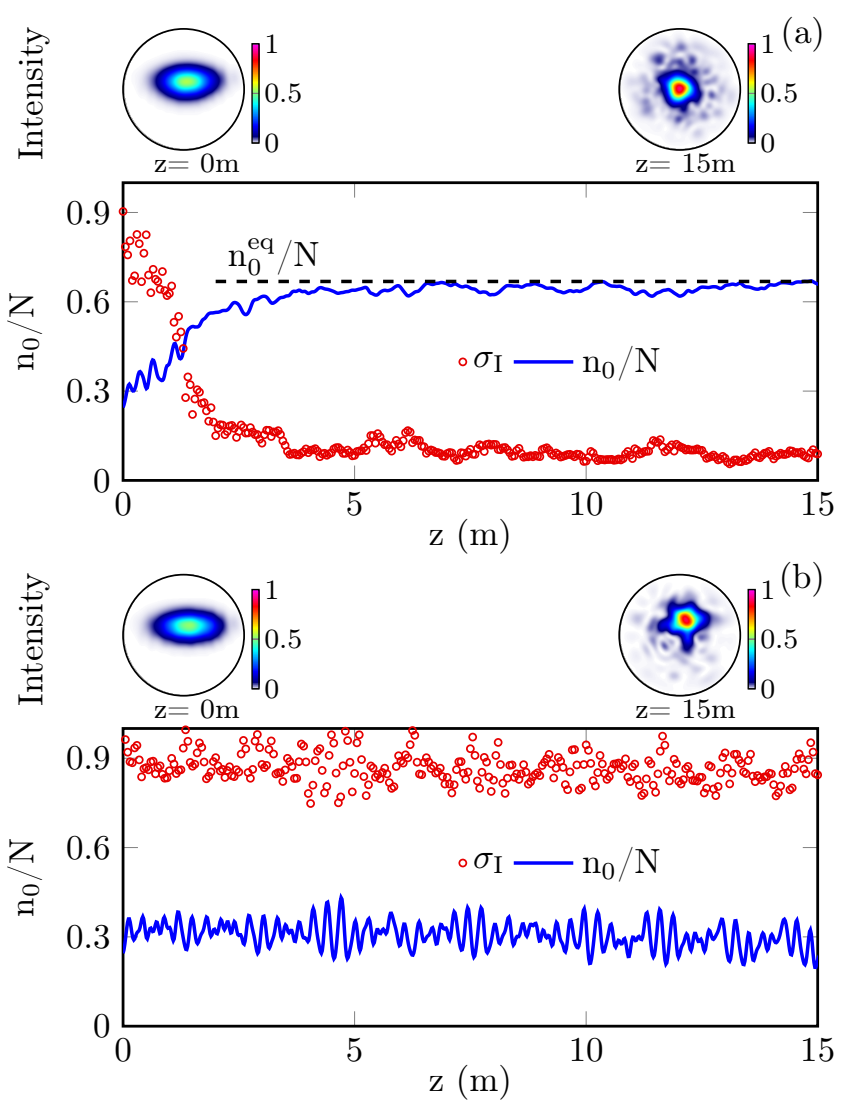

FIG. 3: Disorder-induced beam cleaning: Numerical simulations of the NLS Eq.(8) showing the evolutions of the relative variance of intensity fluctuations $\sigma_{I}(z)$ from Eq.(33) (red circles), and condensate fraction $n_{0}(z) / N$ (blue line), in the presence of disorder (a), and in the absence of disorder (b). The intensity patterns $I(\boldsymbol{r})$ inside the fiber core (circles) are shown at $z=0$ and $z=15 \mathrm{~m}$. The horizontal dashed black line in (a) shows

the condensate fraction at thermal equilibrium, $n_{0}^{e q} / N \simeq 0.68$ (from Eq.(31)). The disorder induces a stable beam cleaning condensation characterized by a significant reduction of intensity fluctuations down to $\sigma_{I} \simeq 0.1$ (a), which is in contrast with the case without disorder where the variance of intensity fluctuations remain almost constant throughout propagation with $\sigma_{I} \simeq 0.9$. Parameters are: $\Delta \beta=2.6 \mathrm{~m}\left(2 \pi / \sigma_{\beta}=2.13 \mathrm{~m}\right.$, $\left.l_{\beta}=30 \mathrm{~cm}\right), N_{*}=120$ modes, $a=26 \mu \mathrm{m}, N=47.5 \mathrm{~kW}$.

ory has been validated even for large condensate fractions in [41]. We recall here the recent works showing that a key assumption of the wave turbulence approach is the existence of a random phase among the modes rather than a genuine Gaussian statistics [86, 87]. 


\section{Disorder-induced beam cleaning}

The amount of 'beam cleaning' can be quantified through the analysis of the fluctuations of the intensity during the propagation in the MMF. To this aim we consider the relative variance of intensity fluctuations relevant for a spatially non-homogeneous incoherent beam

$$
\sigma_{I}^{2}(z)=\frac{\int\left\langle I^{2}(\boldsymbol{r}, z)\right\rangle-\langle I(\boldsymbol{r}, z)\rangle^{2} d \boldsymbol{r}}{\int\langle I(\boldsymbol{r}, z)\rangle^{2} d \boldsymbol{r}},
$$

where the intensity is $I(\boldsymbol{r}, z)=|\boldsymbol{\psi}|^{2}(\boldsymbol{r}, z)$. Note that for a beam with Gaussian statistics we have $\sigma_{I}^{2}=1$. We report in Fig. 3 the evolutions of the variance of intensity fluctuations in the presence and the absence of disorder. The brackets $\langle$.$\rangle in Eq.(33) refers to an averaging over the$ propagation length $\Delta z=10 \mathrm{~mm}$, which is larger than the mode-beating length scale $\sim \beta_{0}^{-1}(\simeq 0.2 \mathrm{~mm}$ in the example of Fig. 3). The simulations in Fig. 3 clearly show that the presence of disorder induces a rapid condensation process, which in turn leads to a significant reduction of the relative standard deviation of intensity fluctuations $\sigma_{I} \simeq 0.1$. Conversely, in the absence of disorder the multimode beam can exhibit an enhanced brightness at some propagation lengths (see the intensity pattern at $z=15 \mathrm{~m}$ in Fig. 3(b)), however its oscillatory multimode nature prevents a stable beam-cleaning propagation, as evidenced by the relative standard deviation of intensity fluctuations that only slowly decreases below $\sigma_{I} \simeq 0.9$.

\section{B. Correlated and partially correlated disorder}

In the previous section III A 1 we have considered a model of disorder that can be termed 'modedecorrelated', in the sense that each individual mode of the MMF experiences a different noise, i.e., the functions $\nu_{p, j}(z)$ in $(9)$ are independent of each other. Although this approach can be considered as justified in different circumstances $[47,51,52]$, one may question its validity for a MMF featured by a large number of modes. In the following we address this question by considering two different models of disorder, namely the fully mode-correlated disorder model, and the partially modecorrelated disorder model.

\section{Mode-correlated noise}

We first consider the fully mode-correlated model that can be considered as the opposite limit of the "modedecorrelated' one, in the sense that all modes experience the same noise (more precisely, the same realization of the noise). In this limit, the $2 \times 2$ matrices describing the modal noise in (9) reduce to $\mathbf{D}_{p}=\mathbf{D}$ with $\mathbf{D}=$ $\sum_{j=0}^{3} \nu_{j} \boldsymbol{\sigma}_{j}$.

The theory developed above for the decorrelated model of disorder can be extended to this fully correlated

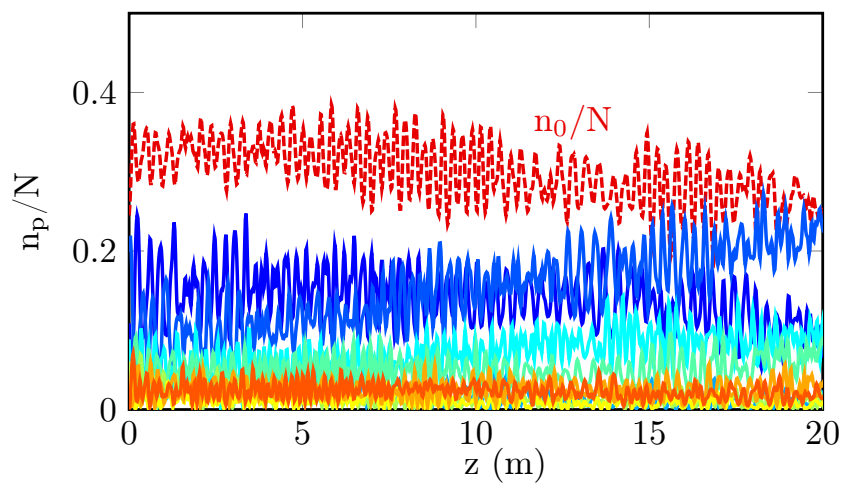

FIG. 4: Mode-correlated disorder: Numerical simulations of the NLS Eq.(8) showing the evolutions of the modal components $n_{p}(z)$, starting from a coherent initial condition: fundamental mode $p=0$ (red dashed), $p=1$ (dark blue solid), $p=2$ (blue solid), $p=3$ (light blue solid), $p=4$ (cyan solid), $p=5$ (light green solid), $p=6$ (green solid), $p=7$ (yellow solid), $p=8$ (orange solid). The mode-correlated disorder does not introduce an effective dissipation in the system $(\Delta \beta=0)$ :

phase-correlations among the low-order modes are preserved and lead to an oscillatory dynamics similar to that in the absence of disorder (see Fig. 1). At complete

thermal equilibrium the system would reach the condensate fraction $n_{0}^{e q} / N \simeq 0.68$ (from Eq.(31)).

Parameters are: $\Delta \beta \simeq 2.6 \mathrm{~m}^{-1}\left(2 \pi / \sigma_{\beta}=2.1 \mathrm{~m}\right.$,

$\left.l_{\beta}=30 \mathrm{~cm}\right)$, the power is $N=47.5 \mathrm{~kW}, N_{*}=120$ modes, $a=26 \mu \mathrm{m}$.

model. The theory reveals in this case a remarkable result, namely that the equations for the fourth-order moments $J_{l m n p}^{(j)}(z)$ do not exhibit an effective damping, i.e., $\Delta \beta=0$, see Appendix A (section IX C). This is in marked contrast with the decorrelated model of disorder discussed above, see Eqs.(16). This result has a major consequence: the fully mode-correlated noise does not modify the regularization of wave resonances and the system recovers a dynamics analogous to that obtained in the absence of any disorder. We illustrate this in Fig. 4 that reports the evolution of the modal components $n_{p}(z)$ obtained by simulation of the modal NLS Eq.(8) in the presence of a mode-correlated disorder. The initial condition is coherent (all modes are correlated with each other) and we see in Fig. 4 that the low-order modes recover an oscillatory dynamics reflecting the presence of strong phase-correlations, as for the coherent regime discussed in the absence of disorder in Fig. 1. This coherent dynamics is consistent with the intuitive idea that, in the absence of an effective dissipation $(\Delta \beta=0)$, the phasecorrelations are not forgotten during the evolution. 


\section{Partially mode-correlated noise}

We have seen that the fully mode-correlated disorder does not introduce an effective dissipation $(\Delta \beta=0)$ and thus leads to a coherent dynamics analogous to that obtained in the absence of disorder. We have thus considered a 'partially correlated' model of disorder in which modes that belong to different groups of degenerate modes experience a decorrelated noise, while degenerate modes of the same group experience the same noise. This artificial model for MMFs can be considered as an intermediate model between the two limits of correlated and decorrelated models. It is mathematically tractable and it illustrates the conjecture that the discrete kinetic Eq.(29) is robust as soon as disorder is not fully modecorrelated.

The theory developed above for the model of decorrelated disorder has been extended to address the partially correlated model, see Appendix A (section IXD). The theory reveals that (second-order) correlations among non-degenerate modes are vanishing small and can be neglected, as it was shown for the model of decorrelated disorder. However, the computation of the fourth-order moments $J_{l m n p}^{(j)}$ is more delicate because the mode-correlated noise introduces more terms in the calculations of the equations for the moments. We obtain different results for the fourth-order moments that depend on the specific modes involved in the moments. Almost all of the fourth-order moments satisfy an evolution equation with a dissipation that is proportional to $\Delta \beta$. This result is analogous to that obtained for the model of decorrelated disorder considered above, though the coefficients in front of $\Delta \beta$ are different and their values depend on the specific modes involved in the fourth-order moment. In addition, at variance with the model of decorrelated disorder, here there are also particular cases where the fourth-order moments do not exhibit any dissipation. Such special cases do not contribute to the fast thermalization process described by the effective dissipation $\Delta \beta$, but instead they induce a reversible exchange of power within a group of degenerate modes, see Appendix A (section IXD).

To summarize, the theoretical developments reported in Appendix A (section IX D) allow us to infer that the kinetic equation is still of the form given by the discrete kinetic Eq.(29) and that the scaling of the rate of thermalization is still given by $L_{k i n}^{\text {disor }} \sim \Delta \beta L_{n l}^{2} / \bar{S}_{l m n p}^{2}$ in Eq.(26). This scaling of the rate of thermalization has been confirmed by the numerical simulations of the NLS Eq.(8). The results are reported in Fig. 5 for the same parameters of disorder as those considered in Fig. 2, except that a model of partial mode-correlated disorder has been considered. The agreement with the discrete kinetic Eq.(29) confirms the scaling (32) of the rate of acceleration of thermalization for the 'partially correlated' model of disorder.

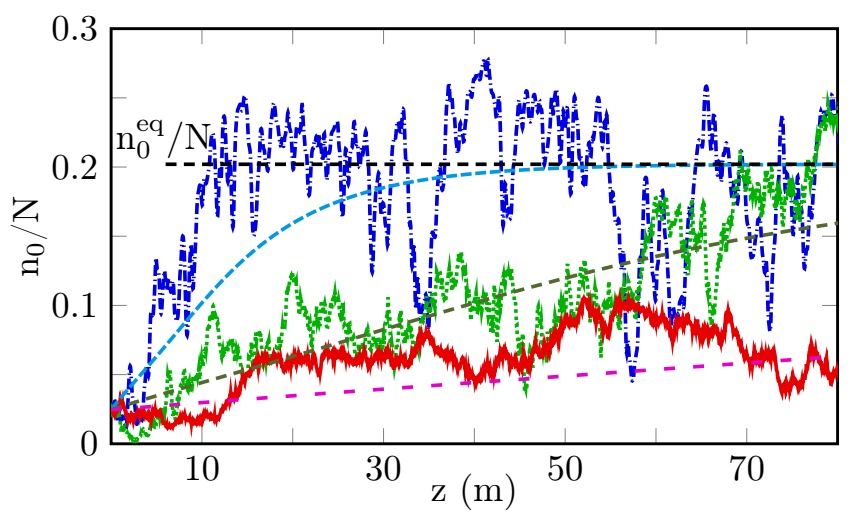

FIG. 5: Scaling of acceleration of thermalization with a partially mode-correlated disorder: Numerical simulations of the NLS Eq.(8) showing the evolutions of the fundamental mode $n_{0}(z)$, for different amounts of disorder $\Delta \beta$. The dashed lines show the corresponding simulations of the discrete kinetic Eq.(29), starting from

the same initial condition as the NLS simulations.

Parameters are: $\Delta \beta \simeq 2.6 \mathrm{~m}^{-1}\left(2 \pi / \sigma_{\beta}=2.1 \mathrm{~m}\right.$, $l_{\beta}=30 \mathrm{~cm}$ ) blue (dashdotted); $\Delta \beta \simeq 10.5 \mathrm{~m}^{-1}$,

$\left(2 \pi / \sigma_{\beta}=26 \mathrm{~cm}, l_{\beta}=1.88 \mathrm{~cm}\right)$ green (dotted); $\Delta \beta \simeq 42 \mathrm{~m}^{-1},\left(2 \pi / \sigma_{\beta}=6.6 \mathrm{~cm}, l_{\beta}=0.47 \mathrm{~cm}\right)$ red (solid); while the power is $N=47.5 \mathrm{~kW}\left(N_{*}=120\right.$ modes, $a=26 \mu \mathrm{m})$. The curves eventually relax to the common theoretical equilibrium value $n_{0}^{e q} / N$ (dashed black line from Eq.(31)) with different rates, and confirm the theoretical scaling of acceleration of condensation in

Eq.(32) for a partially mode-correlated disorder.

\section{STRONG DISORDER}

In this section we discuss the impact of a strong structural disorder, i.e., a noise that couples distinct modes on the evolution of the averaged modal components. More precisely, here we complete the discussion of Ref.[41] on the impact of strong disorder in several respects. We show that a general form of conservative disorder introduces an effective dissipation in the system, which is responsible for an irreversible decay of the first-order moments, as well as an irreversible relaxation of the second-order moments toward different forms of power equipartition among the modes. In this way, we provide a detailed derivation of the kinetic equation accounting for strong disorder at the leading order linear regime, see Appendix C. We also discuss the different regimes described by the considered model of strong disorder, namely the random coupling among polarization components, as well as random coupling among degenerate and non-degenerate modes. In this way, we relate our approach with the corresponding limits described by the so-called Manakov approximation [49-52]. 


\section{A. Model}

Random coupling among the modes can be described by a generalized form of the modal NLS Eq.(8). The random field composed of $N_{*}$ spatial modes is represented by a $2 N_{*}$-dimensional complex valued vector $\boldsymbol{A}(z)=\left(A_{p}(z)\right)_{p=0}^{2 N_{*}-1}$, which is obtained by stacking the $2 N_{*}-$ modal components that include the polarization degrees of freedom. The vector $\boldsymbol{A}(z)$ is governed by the modal NLS equation:

$$
i \partial_{z} \boldsymbol{A}=\boldsymbol{\beta} \boldsymbol{A}+\mathbf{D}^{\mathrm{sd}}(z) \boldsymbol{A}-\gamma \boldsymbol{P}(\boldsymbol{A}),
$$

where $\boldsymbol{\beta}$ is a diagonal matrix with diagonal terms $\beta_{p}$. Random linear coupling between the modes is modelled by the random matrix-valued process $\mathbf{D}^{\text {sd }}(z)$. It consists of a $2 N_{*}$ dimensional generalization of the 2-dimensional model of weak disorder discussed in section III A 1 . The $2 N_{*} \times 2 N_{*}$ Hermitian matrix $\mathbf{D}^{\text {sd }}(z)$ can be written as

$$
\begin{aligned}
\mathbf{D}^{\mathrm{sd}}(z)= & \sum_{q<r} g_{q r}\left(\nu_{q r}(z) \mathbf{H}^{q r}+\mu_{q r}(z) \mathbf{K}^{q r}\right) \\
& +\sum_{q} g_{q} \eta_{q}(z) \mathbf{J}^{q}
\end{aligned}
$$

where the matrices $\mathbf{H}^{q r}, \mathbf{K}^{q r}, \mathbf{J}^{q}$ refer to a $2 N_{*}$ dimensional generalization of the Pauli matrices $\boldsymbol{\sigma}_{j}$ [88]: The matrices $\mathbf{H}^{q r}, \mathbf{K}^{q r}, \mathbf{J}^{q}$ also form a basis for the vector space of $2 N_{*} \times 2 N_{*}$ Hermitian matrices. In this respect, the model (35) can be considered as a general model of strong disorder. For definiteness, the three matrices are given in explicit form in the Appendix C. Following the generalization of the weak disorder model of section III A 1, in the strong disorder model given by Eq.(35) the functions $\eta_{q}$ for $0 \leq q \leq 2 N_{*}-1$, and $\nu_{q r}, \mu_{q r}$ for $0 \leq$ $q<r \leq 2 N_{*}-1$, are zero mean independent and identically distributed Gaussian real-valued random processes, with $\left\langle\eta_{q}(z) \eta_{q}\left(z^{\prime}\right)\right\rangle=\left\langle\nu_{q r}(z) \nu_{q r}\left(z^{\prime}\right)\right\rangle=\left\langle\mu_{q r}(z) \mu_{q r}\left(z^{\prime}\right)\right\rangle=$ $\sigma_{\beta}^{2} \mathcal{R}\left(\frac{z-z^{\prime}}{l_{\beta}}\right)$. We consider Ornstein-Uhlenbeck processes for $\eta_{q}, \nu_{q, r}$ and $\mu_{q, r}$, with $\mathcal{R}(\zeta)=\exp (-|\zeta|) / 2$. The parameters $g_{q r}$ and $g_{q}$ are mode coupling coefficients of order one.

As will be discussed below, the model of disorder (35) allows us to distinguish three different regimes of random mode coupling, namely polarization coupling, coupling among degenerate modes and coupling among nondegenerate modes.

\section{B. Kinetic equation}

In the Appendix $\mathrm{C}$, we derive the equations that govern the evolutions of the first-order moments of the field. The main result obtained by using the Furutsu-Novikov theorem is that the conservative strong disorder introduces an effective dissipation for the evolutions of the average $\langle\boldsymbol{A}\rangle(z)$, which thus exhibits an exponential decay during the propagation, see Eqs.(72-73). This result allows us to study the dynamics of the second-order moments with strong disorder in the regime where linear effects dominate nonlinear effects, as for the weak disorder case considered in section III. However, at variance with weak disorder where the linear dynamics of the second-order moments is trivial (i.e., vanishing correlations, see section III A 2), the presence of strong disorder introduces a non-trivial linear dynamics that dominates nonlinear effects. In the following we study strong disorder at the leading order linear regime.

In Appendix $\mathrm{C}$ we obtain the following equation governing the evolution of the modal components:

$$
\begin{aligned}
\partial_{z} w_{p}= & 2 \sigma_{\beta}^{2} \sum_{m=0}^{2 N_{*}-1} g_{p m}^{2} \int_{0}^{z}\left(w_{m}\left(z^{\prime}\right)-w_{p}\left(z^{\prime}\right)\right) \\
& \times \mathcal{R}\left(\left(z-z^{\prime}\right) / l_{\beta}\right) \cos \left(\left(\beta_{p}-\beta_{m}\right)\left(z-z^{\prime}\right)\right) d z^{\prime}
\end{aligned}
$$

with $w_{p}(z)=\left\langle\left|A_{p}\right|^{2}(z)\right\rangle, p=0, \ldots, 2 N_{*}-1$. Considering that the coupling coefficients do not depend on polarization the $2 N_{*} \times 2 N_{*}$ matrix $g_{p m}$ can be reduced to a $N_{*} \times N_{*}$ matrix $\rho_{p m}$. Furthermore, since $l_{\beta} \ll z, L_{k i n}$, the additional term in the kinetic equation takes the simplified form

$$
\partial_{z} n_{p}=\Delta \beta \sum_{m=0}^{N_{*}-1} \Gamma_{p m} \hat{\mathcal{R}}\left(\left(\beta_{m}-\beta_{p}\right) l_{\beta}\right)\left(n_{m}-n_{p}\right)
$$

where $n_{p}(z)=w_{2 p}(z)+w_{2 p+1}(z)$ is the power in the mode $p\left(=0, \ldots, N_{*}-1\right)$, and $\Gamma_{p m}=\rho_{p m}^{2}$ denotes the mode coupling matrix. The function $\hat{\mathcal{R}}(\kappa)$ denotes the Fourier transform of the correlation function $\mathcal{R}(x)$, which reads $\hat{\mathcal{R}}(\kappa)=1 /\left(1+\kappa^{2}\right)$ for the considered Ornstein-Uhlenbeck process. Note that Eq.(37) has a form similar to that considered to model power cross-talk among different modes in optical telecommunications [44].

\section{Characteristic length scales}

We first note that by adding the extra term (37) in the kinetic equation derived above with weak disorder (see Eq.(29)), the conservation of the energy $E$ is no longer verified, so that a $H$-theorem of the complete kinetic Eq.(29) and (37) would describe an irreversible evolution toward a maximum entropy equilibrium state characterized by an equipartition of power ('number of particles') among the modes. Accordingly, strong disorder would inhibit the self-cleaning condensation process discussed through weak disorder in section III. As will be discussed below (section IVD), this conclusion is not correct when one considers the usual regime of optical beam self-cleaning. In this view, we now discuss the physical meaning of Eq.(37) through the analysis of the model of disorder considered in Eq.(35) and the corresponding different length scales of random mode coupling.

(i) Polarization coupling: The model (35) describes random coupling among the polarizations of a single mode 
that occurs over the propagation length $L_{d} \sim 1 /\left(\Delta \beta \Gamma_{p p}\right)$. Considering a moderate impact of disorder, we can have $\Gamma_{p p} \sim 1$, so that this length scale of polarization random coupling is the same as that considered above in Eq.(11).

(ii) Coupling among degenerate modes: The model (35) describes random coupling within a group of $M_{g}$ degenerate modes over the characteristic propagation length

$$
L_{s d}^{\text {deg }} \sim 1 /\left(M_{g} \Delta \beta \bar{\Gamma}_{g}\right)
$$

where $\bar{\Gamma}_{g}$ is determined by an average of the coupling coefficients $\Gamma_{m p}(m \neq p)$ among the $M_{g}$ modes of the $g$ th mode group. Considering a moderate coupling among the modes $\Gamma_{m p}<\Gamma_{p p}$ for $m \neq p$, we have $L_{d}<L_{s d}^{d e g}$. Physically, the length scale $L_{s d}^{\text {ndeg }}$ represents the typical propagation length such that strong disorder achieves an equipartition of power within each group of degenerate modes. When one considers temporal propagation effects through generalized coupled NLS equations, this effect is known as the Manakov limit of strongly coupled groups of modes, see [50].

(iii) Coupling among non-degenerate modes $\left(\beta_{p} \neq \beta_{m}\right)$ : By Perron-Frobenius theorem, the symmetric matrix $\widetilde{\boldsymbol{\Gamma}}$ defined by

$$
\begin{aligned}
\widetilde{\Gamma}_{m p}= & \Gamma_{m p} \hat{\mathcal{R}}\left(\left(\beta_{p}-\beta_{m}\right) l_{\beta}\right)\left(1-\delta_{m p}^{K}\right) \\
& -\left(\sum_{p^{\prime} \neq m} \Gamma_{m p^{\prime}} \hat{\mathcal{R}}\left(\left(\beta_{p^{\prime}}-\beta_{m}\right) l_{\beta}\right)\right) \delta_{m p}^{K}
\end{aligned}
$$

has a simple zero eigenvalue with the associated unit eigenvector, and all other eigenvalues are negative. As a result, the additional linear coupling terms in the kinetic Eq.(37) tend to redistribute the power amongst all modes, at an exponential rate that can be determined by the second eigenvalue $\lambda_{2}(\widetilde{\boldsymbol{\Gamma}})$ of the matrix $\widetilde{\boldsymbol{\Gamma}}$. The corresponding characteristic length scale is given by

$$
L_{s d}^{n d e g} \sim 1 /\left(\Delta \beta\left|\lambda_{2}(\widetilde{\boldsymbol{\Gamma}})\right|\right)
$$

We recall that $\mathcal{R}(\kappa)$ decays to zero as $\kappa$ goes to infinity, so that $\mathcal{R}\left(\left(\beta_{p}-\beta_{m}\right) l_{\beta}\right)$ is much smaller than $\mathcal{R}(0)=1$ and $L_{s d}^{\text {deg }}<L_{s d}^{n d e g}$. In other words, the length scale $L_{s d}^{n d e g}$ represents the typical propagation length such that strong disorder achieves an equipartition of power among the modes. Considering temporal effects through generalized coupled NLS equations, this effect of power equipartition among all fiber modes is known as the strong coupling regime in the Manakov limit [49, 51].

We finally note that typical values of the three length scales discussed here are estimated in Ref.[45], where complete polarization random coupling is expected to occur over several meters, random mode coupling among degenerate modes over tens meters, and mode coupling among non-degenerate modes over hundreds meters.

\section{Application to beam cleaning: Acceleration of thermalization}

The additional term in the kinetic Eq.(37) provides the characteristic length scales due to strong mode coupling $L_{s d}^{\text {deg }}$ and $L_{s d}^{\text {ndeg }}$. In the usual experiments of beam cleaning we have $\beta_{0} l_{\beta} \gg 1\left(\right.$ since $\beta_{0} \sim 10^{3} \mathrm{~m}^{-1}$ ), so that mode coupling among non-degenerate modes is quenched by the Fourier transform of the correlation function $\hat{\mathcal{R}}\left(\beta_{0} l_{\beta}\right) \ll 1$, i.e., $L_{s d}^{\text {deg }} \ll L_{s d}^{n d e g}$. However, as discussed here above, mode coupling among degenerate modes leads to an exponential relaxation to an equipartition of power within groups of degenerate modes. Unexpectedly, this is a property of the Rayleigh-Jeans distribution, since this equilibrium only depends on the eigenvalue $\beta_{p}$, see the expression of $n_{p}^{e q}$ in Eq.(30). This shows that the impact of strong disorder is not detrimental to achieve wave condensation, but instead it enforces the process of thermalization to the Rayleigh-Jeans distribution, though such an acceleration of thermalization is negligible with respect to the acceleration mediated by weak disorder, see Eq.(32). In addition, considering the short fiber lengths typically used in the experiments of beam self-cleaning ( $L \sim 10-20 \mathrm{~m}$ ), mode coupling among degenerate modes is expected to play a negligible role, $L, L_{k i n}^{\text {disor }} \lesssim L_{s d}^{d e g}$. In the remainder of this article, we will neglect the impact of random mode coupling among degenerate modes.

\section{REDUCED IMPACT OF DISORDER}

The kinetic equations describing the evolution of the modal components $n_{p}(z)$ have been derived under the assumption that disorder effects dominate nonlinear effects $L_{d} \ll L_{n l}$. Although the parameters that characterize the disorder, namely the correlation length $l_{\beta}$ and the effective 'beating length' $2 \pi / \sigma_{\beta}$ (reflecting the 'strength' of disorder) are not precisely known, accurate measurements in single mode optical fibers indicate that such length scales can be of few to several meters [89].

\section{A. Mixed coherent-incoherent regime}

We report in this section numerical simulations of the modal NLS Eq.(8) where disorder and nonlinearity are typically of the same order of magnitude $L_{d} \sim L_{n l}$. For completeness, we consider both models of disorder discussed in section IIIB where the modes experience a decorrelated or a partially correlated noise. Figure 6 reports the results for a decorrelated model of disorder in which we have considered different values of $l_{\beta}$ and $2 \pi / \sigma_{\beta}$ of the noise. We note that, at first sight, for relative small values of $\left(l_{\beta}, 2 \pi / \sigma_{\beta}\right)$, the global evolutions of the modal components $n_{p}(z)$ are similar to those reported in the regime where disorder dominates the nonlinearity. There 


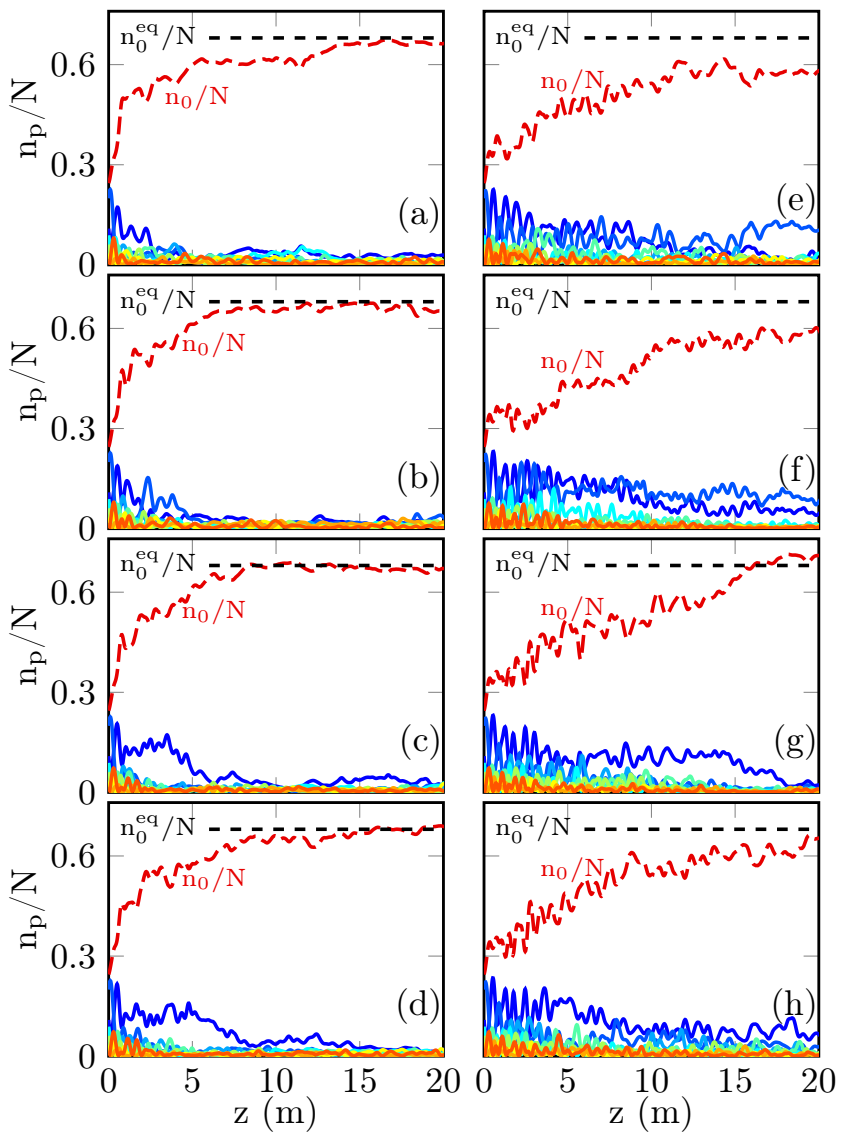

FIG. 6: Mixed coherent-incoherent regime with mode-decorrelated disorder: Numerical simulations of the NLS Eq.(8) showing the evolutions of the modal components $n_{p}(z)$, for different amounts of the strengths of disorder $2 \pi / \sigma_{\beta}$, and correlation lengths $l_{\beta}$ : fundamental mode $p=0$ (red dashed), $p=1$ (dark blue solid), $p=2$ (blue solid), $p=3$ (light blue solid), $p=4$

(cyan solid), $p=5$ (light green solid), $p=6$ (green solid), $p=7$ (yellow solid), $p=8$ (orange solid).

Parameters are: $2 \pi / \sigma_{\beta}=5.3 \mathrm{~m}$ (1st column), $2 \pi / \sigma_{\beta}=10.6 \mathrm{~m}$ (2nd column). 1 st (top) row: $l_{\beta}=0.5 \mathrm{~m}$, 2 nd row: $l_{\beta}=1 \mathrm{~m}$, 3rd row: $l_{\beta}=3 \mathrm{~m}$, 4th row: $l_{\beta}=5 \mathrm{~m}$.

As the impact of disorder is reduced, the modal components enter a mixed coherent-incoherent regime of interaction characterized by a (phase-sensitive) oscillatory behavior of the modal components. At complete thermal equilibrium $n_{0}^{e q} / N \simeq 0.68$ (dashed black line from Eq.(31)). The power is $N=19 \mathrm{~kW}$, the initial condition is a coherent Gaussian beam $\left(N_{*}=120\right.$ modes, $a=26 \mu \mathrm{m})$.

is however a difference that distinguishes the two regimes. The rapid fluctuations on the small length scale of disorder $L_{d}\left(\ll L_{n l}\right)$ of Figs. 2-5 get smoother in the regime where disorder and nonlinearity are of the same order in Fig. 6. Also remark that a variation of the correlation length $l_{\beta}$ (different lines in Fig. 6) has a marginal impact
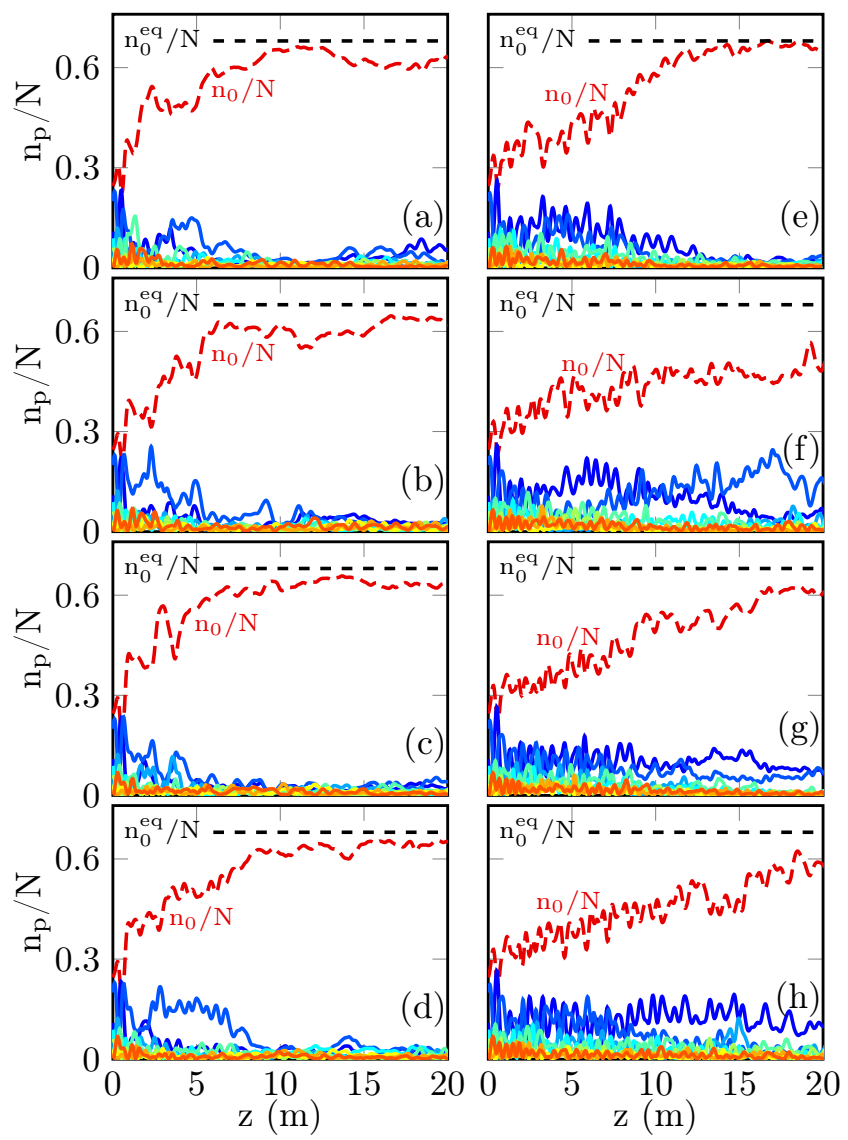

FIG. 7: Mixed coherent-incoherent regime with partially mode-correlated disorder: Numerical simulations of the NLS Eq.(8) showing the evolutions of the modal components $n_{p}(z)$, for different amounts of the strengths of disorder $2 \pi / \sigma_{\beta}$, and correlation lengths $l_{\beta}$. Parameters and initial condition are the same as in Fig. 6, except that a partially mode-correlated disorder has been considered.

on the dynamics as compared to the strength of disorder $2 \pi / \sigma_{\beta}$ (columns in Fig. 6 ) - note that the values of $2 \pi / \sigma_{\beta}$ in Fig. 6 correspond to refractive index fluctuations of the order $\delta n \sim \sigma_{\beta} / k_{0} \sim 10^{-7}$.

It is interesting to note that, by increasing further the correlation and beating lengths of disorder $\left(l_{\beta}, 2 \pi / \sigma_{\beta}\right)$, the system enters a different regime, which is characterized by the presence of pronounced oscillations of the modal components. This oscillatory behavior reflects the presence of a phase-correlation among the modal components, as it was discussed through the coherent modal regime of interaction in the absence of any disorder in section II B. Here, the presence of a moderate disorder is not sufficient to remove such coherent phase-correlation dynamics, so that the modes exhibit a mixed coherentincoherent regime of interaction.

The same phenomenological behavior about the impact of disorder on the system is observed by consider- 
ing a partially correlated model of noise, as illustrated in Fig. 7. Interesting to note, for moderate values of disorder the evolutions of the modal components is very similar to that observed for a decorrelated model of disorder (compare the first columns in Fig. 6 and Fig. 7). The fact that a partial correlation among the modes does not alter the rate of thermalization was already discussed in the regime where disorder dominates the nonlinearity (see section IIIB). Actually, the main difference between the decorrelated and partially correlated models of disorder is observed by further reducing the impact of disorder (i.e., by further increasing $l_{\beta}$ and $2 \pi / \sigma_{\beta}$ ). In this case, the partially correlated noise model leads to a more pronounced oscillatory behavior, a feature that can easily be interpreted by remarking that since the degenerate modes see the same noise, the impact of disorder is less efficient in breaking the phase-correlations among the modes. As a result, when one considers the partially mode-correlated noise for large values of $l_{\beta}$ and $2 \pi / \sigma_{\beta}$, the mixed coherent-incoherent regime of interaction becomes clearly apparent, and the corresponding oscillatory behavior of the modal components leads to a deceleration of the thermalization process, see the second column of Fig. 7. We note that the simulations in Figs. 6-7 have been realized with the parameters of the recent experiment of Ref.[41] (in particular the same power). The simulations then appear consistent with the experimental results - for instance a propagation length similar to that of the fiber length used in Ref.[41] (i.e. $\sim 12 \mathrm{~m}$ ) is sufficient to evidence a significant process of condensation starting from a coherent initial condition.

We finally comment the impact of a mode-correlated model of disorder. We have seen in section III B that in the regime $L_{d} \ll L_{n l}$ this model of disorder does not introduce an effective dissipation so that it does not lead to a fast process of condensation. In the regime $L_{d} \sim L_{n l}$ the simulations still reveal a persistent oscillatory behavior of the modes by starting from a coherent initial condition (as in Figs. 6-7), while a significant deceleration of condensation featured by a mixed coherent-incoherent regime has been observed by starting the simulations from a speckle beam.

\section{B. Polarization effects}

In this section we discuss the polarization dynamics of the modal components during their nonlinear propagation through the MMF in the presence of disorder effects. We consider the usual definition of the Stokes vectors integrated over the transverse spatial section of the optical beam $S^{(0)}(z)=$ $\int\left|\psi_{x}(\boldsymbol{r}, z)\right|^{2}+\left|\psi_{y}(\boldsymbol{r}, z)\right|^{2} d \boldsymbol{r}, S^{(1)}(z)=\int\left|\psi_{x}(\boldsymbol{r}, z)\right|^{2}-$ $\left|\psi_{y}(\boldsymbol{r}, z)\right|^{2} d \boldsymbol{r}, \quad S^{(2)}(z)=2 \operatorname{Re} \int \psi_{x}(\boldsymbol{r}, z)^{*} \psi_{y}(\boldsymbol{r}, z) d \boldsymbol{r}$, $S^{(3)}(z)=-2 \operatorname{Im} \int \psi_{x}(\boldsymbol{r}, z)^{*} \psi_{y}(\boldsymbol{r}, z) d \boldsymbol{r}$. By expanding

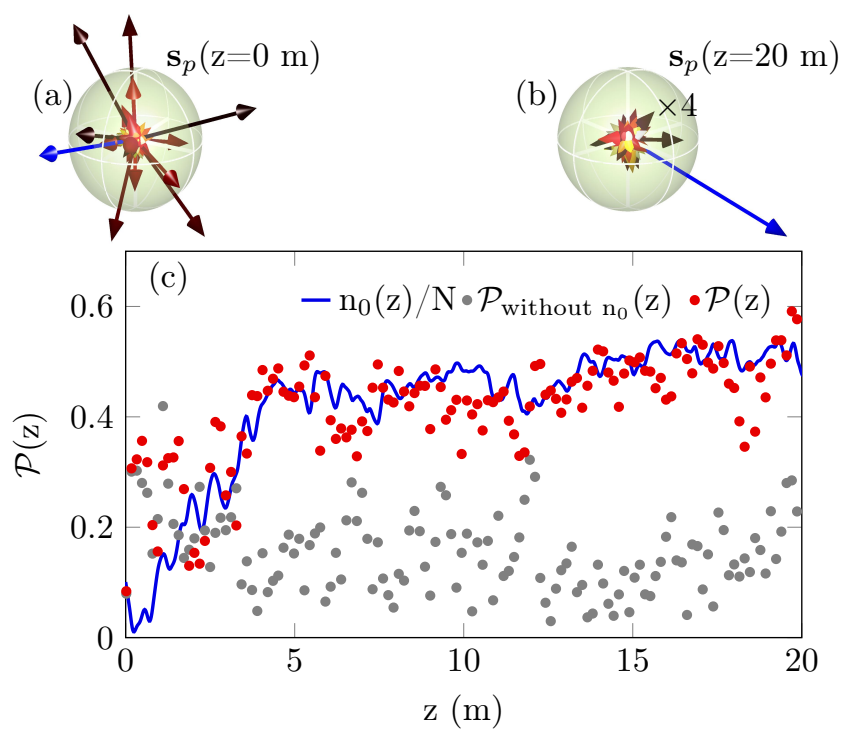

FIG. 8: Evolution during the propagation $z$ of the effective degree of polarization $\mathcal{P}$ computed by taking into account the contribution of the fundamental mode

(red points), and without including its contribution

(grey points) (c). Corresponding evolution of the condensate amplitude $n_{0}(z)$ (blue line) (c). The initial condition is a speckle beam, so that the Stokes vectors of the modal components are not aligned with each other (a), leading to a small $\mathcal{P}(z=0)$. During the propagation, the condensation process entails an effective repolarization of the beam because $\left|\left\langle s_{0}\right\rangle\right| \gg\left|\left\langle s_{p \neq 0}\right\rangle\right|(\mathrm{a}-\mathrm{b})$ - to improve their visibility the lengths of the Stokes vectors $\left\langle\boldsymbol{s}_{p \neq 0}\right\rangle$ have been multiplied by a factor $\times 4$ in (b). Parameters: $l_{\beta}=30 \mathrm{~cm}, 2 \pi / \sigma_{\beta}=2.1 \mathrm{~m}, N=47.5 \mathrm{~kW}\left(N_{*}=120\right.$ modes, $a=26 \mu \mathrm{m})$.

the field over the modes, we have

$$
\begin{aligned}
& S^{(0)}(z)=\sum_{p=0}^{N_{*}-1}\left|A_{p, x}(z)\right|^{2}+\left|A_{p, y}(z)\right|^{2}=\sum_{p=0}^{M-1} s_{p}^{(0)}, \\
& S^{(1)}(z)=\sum_{p=0}^{N_{*}-1}\left|A_{p, x}(z)\right|^{2}-\left|A_{p, y}(z)\right|^{2}=\sum_{p=0}^{M-1} s_{p}^{(1)}, \\
& S^{(2)}(z)=2 \operatorname{Re} \sum_{p=0}^{N_{*}-1} A_{p, x}(z)^{*} A_{p, y}(z)=\sum_{p=0}^{N_{*}-1} s_{p}^{(2)}, \\
& S^{(3)}(z)=-2 \operatorname{Im} \sum_{p=0}^{N_{*}-1} A_{p, x}(z)^{*} A_{p, y}(z)=\sum_{p=0}^{N_{*}-1} s_{p}^{(3)} .
\end{aligned}
$$

It is important to recall that our model does not account for the temporal dynamics of the optical wave. Accordingly, we resort to a definition of an effective degree of polarization $\mathcal{P}(z)=\sqrt{\sum_{j=1}^{3}\left\langle S^{(j)}\right\rangle^{2}} /\left\langle S_{0}\right\rangle$, by considering an average $(\langle\cdot\rangle)$ over the evolution $z$-variable. As 
is well-known, caution should be exercised with the notion of degree of polarization, which is inherently related to the underlying averaging procedure. In this respect, we note that for an averaging length $\Delta z$ much larger than the correlation lengths $l_{\beta}, 2 \pi / \sigma_{\beta}$, the degree of polarization vanishes $\mathcal{P} \simeq 0$. This results from the theory developed in Appendix A (section IX A), where we have shown that in the regime $L_{d} \ll L_{n l}$ the correlations among the orthogonal polarization components are vanishingly small. To study the correlations $\left\langle S^{(j)}\right\rangle$, here we consider a moderate value of the spatial averaging $\Delta z \simeq 15 \mathrm{~cm}$.

We report in Fig. 8 the evolution of $\mathcal{P}(z)$ starting from a random distribution of the modal components with random phases ('speckle' beam). Accordingly, the initial Stokes vectors of the modes $\boldsymbol{s}_{p}$ are not aligned with each other, i.e. their random orientation leads to a vanishing $\operatorname{sum}\langle\boldsymbol{S}\rangle=\sum_{p}\left\langle\boldsymbol{s}_{p}\right\rangle \simeq 0$ and thus $\mathcal{P}(z=0) \simeq 0$. As the beam propagates through the MMF, it undergoes a beam-cleaning condensation in which the evolution of $\mathcal{P}(z)$ follows a behavior similar to that of the condensate fraction $n_{0}(z) / N$. This is because the modal distribution gets dominated by the macroscopic population of the fundamental mode:

$$
\left|\left\langle s_{0}\right\rangle\right| \gg\left|\left\langle s_{p}\right\rangle\right| \text { for } p \neq 0
$$

where $|\cdot|$ denotes the modulus of the vector. According to (42) the sum over the modal Stokes vectors no longer vanishes, $\langle\boldsymbol{S}\rangle=\sum_{p}\left\langle\boldsymbol{s}_{p}\right\rangle \neq 0$. Note that the effective degree of polarization does not increase if $\mathcal{P}(z)$ is computed without including the contribution of the fundamental mode (grey points in Fig. 8(c)), which confirms that the repolarization of the beam is a consequence of wave condensation.

Let us now consider the case where the launched optical beam is spatially coherent and polarized, as for typical experiments of beam self-cleaning. The numerical simulation corresponding to this initial condition is reported in Fig. 9(a) for a moderate disorder $L_{d} \gtrsim L_{n l}$. In this case, all of the Stokes vectors are initially aligned, so that $\mathcal{P}(z=0)=1$. For short propagation lengths, the disorder scrambles the phase relation-ship among the modes, thus leading to a misalignment of the Stokes vectors and then to a partial depolarization of the beam. Subsequently, the effective repolarization induced by condensation leads to a saturation of the decrease of $\mathcal{P}(z)$.

In this regime of beam cleaning where disorder effects are moderate $L_{d} \gtrsim L_{n l}$, the fundamental mode is macroscopically populated and its polarization dynamics is not significantly altered by disorder nor by the coupling to other modes. As a consequence, the polarization dynamics of the fundamental mode exhibits a well-known effect of nonlinear polarization rotation on the Poincare sphere [77], as can be observed in Fig. 9(b). This effect is characterized by a rotation of the Stokes vector in the $\left(s_{0}^{(1)}, s_{0}^{(2)}\right)$ plane with an ellipticity that can remain almost constant over some propagation lengths, $s_{0}^{(3)}=$ const. Note that

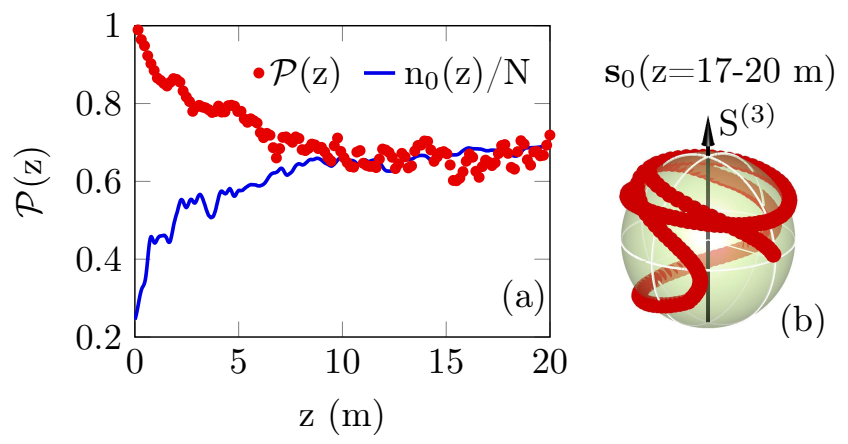

FIG. 9: (a) Evolution during the propagation $z$ of the effective degree of polarization $\mathcal{P}$ (red points), and condensate fraction $n_{0}(z) / N$ (blue line). The initial condition is a polarized coherent optical beam, i.e., the Stokes vectors of the modal components are aligned with each other, leading to $\mathcal{P}(z=0)=1$. During the propagation, the disorder misaligns the Stokes vectors leading to a partial depolarization of the beam. Then the condensation-induced repolarization effect leads to a saturation of the decrease of $\mathcal{P}(z)$. (b) Trajectory of the

Stokes vector $\boldsymbol{s}_{0}(z)$ on the Poincaré sphere over the propagation length $17 \mathrm{~m}$ to $20 \mathrm{~m}$, showing an effect of nonlinear polarization rotation. Parameters: $l_{\beta}=5 \mathrm{~m}$, $2 \pi / \sigma_{\beta}=25 \mathrm{~m}, N=47.5 \mathrm{~kW}\left(N_{*}=120\right.$ modes,

$$
a=26 \mu \mathrm{m}) \text {. }
$$

this nonlinear polarization rotation of the self-cleaned optical beam has been observed experimentally in Ref.[54]. To interpret their results, the authors of [54] invoke the key impact of the temporal profile of the optical pulse injected in the MMF. Indeed, the frequency rotation of the Stokes vector depends on the instantaneous value of the power, so that different power levels of the injected pulse profile exhibit different rotation speeds of the Stokes vector. Accordingly, a quantitative comparison between the experimental results of Ref.[54] and our numerical results is not possible without including temporal effects in our model, and specifically the temporal profile of the injected optical pulse.

We followed the experimental procedure of Ref.[54] by analyzing the polarization properties in the transverse spatial distribution of the optical beam. The effective degree of polarization $\mathcal{P}(x, y=0)$ reported in Fig. 10 is computed by performing a spatial integration over a diaphragm of same diameter as the fundamental mode of the MMF $(9 \mu \mathrm{m})$, which is moved across the beam along the $x$-axis by keeping fixed the $y=0$ position. As expected, the transverse profile of the degree of polarization exhibits a bell-shaped profile indicating a significant repolarization of the self-cleaned beam. This confirms that the effective repolarization of the beam discussed here results from the process of condensation and the associated macroscopic population of the fundamental mode of the MMF. 


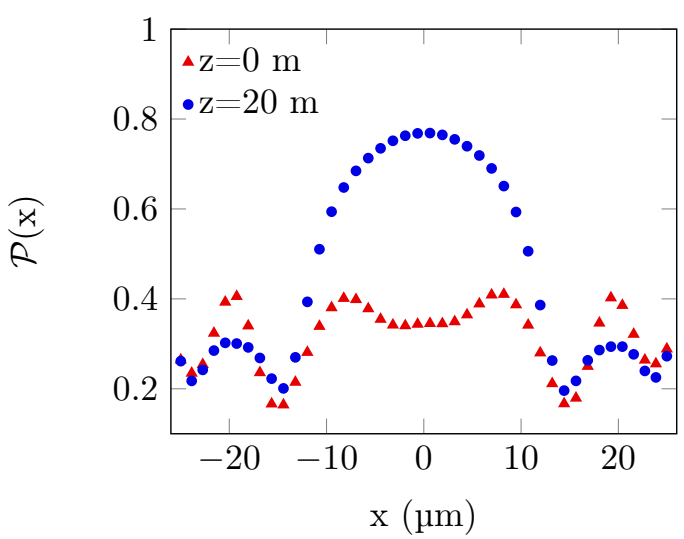

FIG. 10: Polarization properties across the transverse surface section of the self-cleaned beam reported in Fig. 8: Effective degree of polarization $\mathcal{P}$ computed along the $x$-axis by keeping fixed the $y$-position $(y=0)$, at $z=0 \mathrm{~m}$ (red triangles), and $z=20 \mathrm{~m}$ (blue dots). As a consequence of wave condensation, the central part of the beam exhibits a significant repolarization process. Parameters: $l_{\beta}=30 \mathrm{~cm}$, $2 \pi / \sigma_{\beta}=2.1 \mathrm{~m}, N=47.5 \mathrm{~kW}\left(N_{*}=120\right.$ modes, $a=26 \mu \mathrm{m})$.

\section{FREEZING AND SLOWING-DOWN OF THERMALIZATION}

In this section we discuss an important consequence of the discrete nature of wave turbulence in MMFs, namely an effective freezing of the process of thermalization and condensation, which should also explain why optical beam cleaning has not been observed in stepindex MMFs. In addition, we comment the impact on the turbulent dynamics of a perturbation of the dispersion relation, which is shown to modify the regularization of wave resonances and then the rate of thermalization of the optical beam.

\section{A. Absence of beam-cleaning in step-index fibers: Freezing of thermalization}

The theory developed in this article can be applied in principle to different waveguide geometries, such as stepindex MMFs that are characterized by a homogeneous circular potential $V(r)$. This example is important in that the effect of optical beam self-cleaning has not been observed in step index MMFs.

To discuss this experimental observation, we first note that at variance with a GRIN MMF, in a step-index MMF the eigenvalues $\beta_{p}$ are not equally spaced and degenerate modes are scarce. We recall in this respect that thermalization takes place through the excitation of higher-order modes, a feature that essentially occurs by "nontrivial" resonances, i.e., resonances that involve at least three (or four) non-degenerate modes. It is important to note that, at variance with a GRIN fiber, in step-index MMF such nontrivial resonances are not exact resonances verifying $\Delta \omega_{l m n p}=0$. According to our discussion on discrete vs continuous wave turbulence (see section III A 4-III A 5), this means that incoherent light propagation in step-index MMFs should be described by quasi-resonances through a continuous wave turbulence approach. However, as will be shown below quasiresonances are poorly efficient in actual step-index MMFs and many of them verify $\left|\Delta \omega_{l m n p}\right|>1 / L_{n l}$, i.e., the frequency mismatch is too large to provide a non-vanishing contribution to the kinetic equation.

\begin{tabular}{|c|c|c|c|c|}
\hline & $N_{\text {res }}^{(1)}$ & $\chi_{\text {eff }}^{(1)}$ & $N_{\text {res }}^{(2)}$ & $\chi_{\text {eff }}^{(2)}$ \\
\hline Step-index & 1376 & 4.7 & 12256 & 15 \\
\hline GRIN & 8369504 & 630 & 8369504 & 630 \\
\hline
\end{tabular}

TABLE I: Number of quasi-resonances $N_{\text {res }}^{(1,2)}$ and corresponding efficiencies $\chi_{\text {eff }}^{(1,2)}=\sum_{l m n p} S_{l m n p}^{2}$ for a stepindex MMF for $L_{n l}=1 \mathrm{~m}$ and for $L_{n l}=25 \mathrm{~cm}$, respectively. For a GRIN MMF, $N_{\text {res }}$ denote the number of exact resonances. The significant reduction of $N_{\text {res }}^{(j)}$ and $\chi_{\text {eff }}^{(j)}$ for the step-index MMF with respect to the GRIN MMF is responsible for an effective freezing of the process of thermalization and condensation.

We illustrate this by comparing the number of nontrivial resonances $N_{\text {res }}$ for a GRIN and a step-index MMF - more specifically we compute the number of exact resonances for the GRIN fiber $\left(\Delta \omega_{l m n p}=0\right)$, and the number of quasi-resonances for the step-index fiber $\left|\Delta \omega_{l m n p}\right| \ll L_{n l}^{-1}$. The resonances are non-trivial in the sense that they involve at least three different groups of non-degenerate modes. We considered fibers with approximatively the same number of modes: $N_{*}=120$ for the GRIN $\left(n_{0}=1.47, n_{1}=1.457, a=26 \mu \mathrm{m}\right), N_{*}=121$ for the step-index fiber $\left(n_{0}=1.4496, n_{1}=1.4462\right.$, $a=37 \mu \mathrm{m})$. We also considered different values of the nonlinear length $L_{n l}=25 \mathrm{~cm}$ and $L_{n l}=1 \mathrm{~m}$, and computed the number of quasi-resonances in the step-index fiber with the criterion $\left|\Delta \omega_{l m n p}\right| \leq L_{n l}^{-1} / 10$. The results are reported in Table I, which show a drastic reduction of the number of resonances and corresponding efficiencies in the step-index fiber as compared to the GRIN fiber.

To summarize, incoherent light propagation in a stepindex MMF is not described by a discrete turbulence regime because of the absence of exact non-trivial resonances. The step-index MMF also exhibits poorly efficient quasi-resonances that essentially freeze the development of a continuous turbulence regime. Note that the number of quasi-resonances contributing to the kinetic equation can be increased by considering larger radii of step-index MMFs, thus leading to a reduced mode spacing. In this case an efficient continuous turbulence regime can be established in principle (provided that sufficient power is launched in the fiber). However, as discussed in section III A 4, in this case the impact of weak disorder 
is expected to prevent the conservation of kinetic energy, which would thus inhibit wave condensation.

\section{B. Freezing discrete turbulence and thermalization with specific initial conditions}

The wave turbulence theory developed in this paper is relevant when the optical beam populates many modes of the MMF. This is the case for instance for the experiments reported in [41], in which the optical beam is passed through a diffuser to degrade its transverse wavefront profile before injection into the MMF. It has been shown in this case that, by increasing the excitation of modes, the effect of beam self-cleaning is degraded, as described by wave condensation and the equilibrium condensation curve, see [41]. It is important to stress, however, that there exist particular conditions of beam injection into the MMF that can excite only few modes of the fiber, as it has been recently reported in Refs.[67, 90]. Consider for instance the case where a Gaussian beam with a radius comparable to that of the fundamental mode is injected at perfect normal incidence and exactly at the center of the MMF. In this case only few radial modes are excited, while all modes featured by a non-homogeneous azimuthal profile are not excited at all. Another important example reported in Ref. [67] is the excitation with a Gaussian beam whose incident external angle is adjusted around 2.5 degrees, in order to excite the fiber beyond the numerical aperture of the fundamental mode. In this way, the amount of power coupled into the fundamental mode is limited, while a high fraction of power results to be coupled into the $\mathrm{LP}_{11}$ mode. With this specific initial condition, the experiments in Ref. [67] reported a remarkable effect of beam self-cleaning upon power on this preferentially excited mode $\mathrm{LP}_{11}$ mode.

This latter observation may appear at first sight in contradiction with the effect of condensation and thermalization to the RJ distribution. However, as discussed all along this paper, incoherent light propagation in MMFs is described by the discrete wave turbulence regime that is dominated by exact resonances. Considering the small number of modes excited with the above specified initial conditions, we shall see that the processes of thermalization and condensation result to be essentially frozen. Indeed, following the discussion about the absence of beam cleaning in step-index MMFs (section VIA), here the same argument of freezing of thermalization should explain why condensation of power into the fundamental mode is not observed with the specific tilted injection favouring the excitation of the $\mathrm{LP}_{11}$ mode [67]. In the same way, for perfect normal incidence injection of a small Gaussian beam, the numerical simulations do not evidence the establishment of a RJ equilibrium state featured by energy equipartition among the modes within the short fiber length used in the experiments. This is a consequence of the discrete nature of the resonances manifold in MMFs, which exhibits clusters of resonant mode interactions in relation with finite size effects in discrete wave turbulence [7, 55-66].

\begin{tabular}{|c|c|c|c|c|}
\hline mode excitation & $N_{\text {res }}^{(1)}$ & $\chi_{\text {eff }}^{(1)}$ & $N_{\text {res }}^{(2)}$ & $\chi_{\text {eff }}^{(2)}$ \\
\hline$($ i $)$ & 18380 & 7.4 & 93840 & 16 \\
\hline (ii) & 11600 & 10.7 & 229776 & 46 \\
\hline (iii) & 96840 & 67 & 1716000 & 283 \\
\hline (iv) & 922944 & 210 & 8369504 & 603 \\
\hline
\end{tabular}

TABLE II: Number of resonances $N_{\text {res }}^{(1,2)}$ and corresponding efficiencies $\chi_{\mathrm{eff}}^{(1,2)}=\sum_{l m n p} S_{l m n p}^{2}$ for two different values of kinetic energies $E^{(1)} / E_{\text {crit }}=0.32$ (columns $2-3)$ and $E^{(2)} / E_{\text {crit }}=0.54$ (columns $4-5$ ). The initial conditions (i) and (ii) refer to particular injection conditions: (i) coherent Gaussian beam at perfect normal incidence; (ii) coherent Gaussian beam with a specific tilt angle favouring the excitation of the $\mathrm{LP}_{11}$ mode (see Ref.[67]). The initial conditions (iii) and (iv) refer to generic incoherent (speckle-like) beams with exponential (iii), Lorentzian (iv), spectral distributions in mode space. The number of resonances and their efficiencies are considerably smaller for the particular excitations (i) and (ii) as compared to the generic states (iii)-(iv). Since the energy $E^{(1)}$ (or $\left.E^{(2)}\right)$ is kept fixed, the corresponding 'amount of disorder' is the same for all initial conditions (i)-(iv): The significant reduction of $N_{\text {res }}^{(j)}$ and $\chi_{\text {eff }}^{(j)}$ for the particular states (i)-(ii) reflects the peculiar modal excitation due to the specific injection conditions into the MMF.

We have computed the number of non-trivial resonances $N_{\text {res }}$ (involving at least three groups of nondegenerate modes) and their corresponding efficiencies $\chi_{\mathrm{eff}}=\sum_{l m n p} S_{l m n p}^{2}$ for the particular initial conditions discussed above, namely: (i) Gaussian beam at normal incidence; (ii) Gaussian beam with a specific tilt angle (2.5 degrees) favouring the excitation of the $\mathrm{LP}_{11}$ mode. This computation has been realized for two different values of the kinetic energy $E^{(1)}$ and $E^{(2)}$, corresponding to a Gaussian beam with $\mathrm{FWHM}=2 \mathrm{FWHM}_{0}$ for $E^{(1)}$, and $\mathrm{FWHM}=3 \mathrm{FWHM}_{0}$ for $E^{(2)}, \mathrm{FWHM}_{0}$ being the fullwidth-half-maximum of the fundamental mode. The results are reported in Table II for the values of the energies $E^{(1)} / E_{\text {crit }}=0.32$ (columns $2-3$ ) and $E^{(2)} / E_{\text {crit }}=0.54$ (columns 4-5), where $E_{\text {crit }}=N V_{0}\left(1+2 \beta_{0} / V_{0}\right) / 2$ denotes the critical energy of the transition to condensation [17]. We have compared these results with a generic incoherent (speckle-like) beam, which is characterized by an exponential or a Lorentzian spectral distribution in mode space (see (iii)-(iv) in Table II). A resonance is retained in the computation whenever the modal amplitudes are larger than some threshold value $\left(n_{p} \geq 0.1 \%\right.$ in Table II, with $\left.\sum_{p} n_{p}=1\right)$. The main result is that $N_{\text {res }}$ and $\chi_{\text {eff }}$ decrease in a substantial way for the particular initial conditions (i)-(ii) where a small Gaussian beam is injected either at perfect normal incidence or with a specific tilt angle favouring the excitation of the $\mathrm{LP}_{11}$ mode. It is important to stress that this comparison is realized 
for the same kinetic energy $E$, i.e., the same 'amount of disorder' in the initial condition. Accordingly, the significant reduction of $N_{\text {res }}$ and $\chi_{\text {eff }}$ for the particular initial conditions (i)-(ii) reveals the specificity of such modal excitation as compared to the generic initial conditions (iii)-(iv). We note that such specific initial conditions are commented by the authors of Refs. [67, 90], who state that beam-cleaning on the $\mathrm{LP}_{11}$ mode requires "a lengthy and difficult to exactly reproduce manual procedure, i.e., with a tricky adjustment of tilted launching conditions".

To conclude this discussion, we have seen in section II B that typical MMFs used in beam-cleaning experiments behave as a dynamical system with a limited number of degrees of freedom, in relation with the discrete nature of wave turbulence in MMFs. When many modes are excited through generic initial conditions in the presence of a significant amount of structural disorder $\left(L_{d} \ll L_{n l}, z\right)$, the system exhibits a well developed discrete turbulence regime that is accurately described by the discrete kinetic equation derived here. On the other hand, for specific initial conditions characterized by the excitation of a small number of modes, then the discrete structure of the resonance manifold of MMFs can freeze the thermalization and thus the condensation processes within the short fiber lengths considered in the experiments (5$8 \mathrm{~m}$ in $[67,90])$. The derived kinetic equations should not be relevant to describe this regime of few-mode interaction over small propagation lengths. In particular, the impact of disorder may be considered as perturbative in this regime where the system can exhibit a partially phase-sensitive coherent interaction, which may probably be described by the tools developed to study finite size effects in turbulence, such as discrete and mesoscopic wave turbulence and the associated clusters of resonant mode interactions $[7,55-66]$. Such a partially coherent few-mode interaction regime can be mastered by a fine tuning of the transverse profile of the laser excitation $[67,90]$. In the recent experiments [90] beam cleaning of many low-order modes has been reported owing to an ingenious feedback-induced adaptive profiling of the transverse wavefront phase of the coherent beam excitation (also see [91]). More precisely, owing to a feedback loop, the transverse wavefront phase of the injected beam is adjusted by an iterative procedure so as to force the beam to convergence toward a pre-established target mode at the fiber output. This adaptively controlled cleaning of low-order modes is of different nature than the spontaneous phenomenon of condensation on the fundamental mode resulting from the natural process of thermalization toward the equilibrium distribution.

\section{Corrections of the dispersion relation}

The wave turbulence approach developed in this article is based on the NLS equation with an ideal parabolic potential and the (linear) dispersion relation $\beta_{p}=\beta_{0}\left(p_{x}+\right.$ $\left.p_{y}+1\right)$. Several factors introduce perturbations to this expression of the dispersion relation, which we write in the form $\tilde{\beta}_{p}=\beta_{p}+b_{p}$, where the perturbation $b_{p}$ is a function of $\left(p_{x}, p_{y}\right)$ with $b_{0} / \beta_{0} \ll 1$. An example of perturbation is provided by the well known fact that a GRIN MMF usually exhibits deviations from the ideal parabolic shape, i.e., $V(\boldsymbol{r}) \sim|\boldsymbol{r}|^{\nu}$ with an exponent that deviates from $\nu=2$. The general expression of the eigenvalue is rather complicated and of the form $\tilde{\beta}_{p} \propto\left(1+p_{x}+p_{y}\right)^{2 \nu /(\nu+2)}$ [92]. Considering a deviation of a few percents from $\nu=2$ [93], one has $b_{0} / \beta_{0} \sim 5 \times 10^{-3}$ with usual parameters of beam cleaning experiments. An other example is the leading order correction due to angular dispersion effects in the Helmholtz equation, $b_{p}=\left(\beta_{0}^{2} /\left(2 k_{0} n_{0}\right)\right)\left(1+p_{x}+p_{y}\right)^{2}$, which gives $b_{0} / \beta_{0} \sim 2 \times 10^{-4}$ with usual beam-cleaning experimental parameters. In addition, we can notice that the truncation of the parabolic refractive index profile due to the presence of the fiber cladding introduces significant perturbations of the higher-order eigenvalues (see Fig. 5 in Ref.[17]). It is also important to note that the standard deviation of the fluctuations of the structural disorder of the MMF due to imperfections and external perturbations (term $\mathbf{D}_{p} \boldsymbol{A}_{p}$ in the modal NLS Eq.(8)) may be of the same order as the correction of the dispersion relation. In this respect, we also recall that a bending of the fiber introduces a correction in the propagation constant of the order $\simeq a / R_{c}$, where $a$ is the fiber radius and $R_{c}$ the radius of the bending $\left(a / R_{c} \simeq 10^{-4}\right.$ with $a \simeq 25 \mu \mathrm{m}$ and $R_{c} \simeq 25 \mathrm{~cm}$ ).

Let us discuss on the possible impact of perturbations of the dispersion relations. In this respect, resonances that are exact at leading order $\left(\Delta \omega_{l m n p}=\beta_{l}+\beta_{m}-\beta_{n}-\right.$ $\left.\beta_{p}=0\right)$ exhibit a residual non-resonant contribution, i.e., $\Delta \tilde{\omega}_{l m n p}=\tilde{\beta}_{l}+\tilde{\beta}_{m}-\tilde{\beta}_{n}-\tilde{\beta}_{p}=\Delta b_{l m n p}$ with $\Delta b_{l m n p}=b_{l}+$ $b_{m}-b_{n}-b_{p}$. We derive the kinetic equation accounting for the correction on the dispersion relation with the above assumption $L_{d}=1 / \Delta \beta \ll L_{n l}<L_{k i n}^{\text {disor }}$. The result of the convolution integral Eq.(18) is approximated by

$$
J_{l m n p}^{(j)} \simeq \gamma\left\langle Y_{l m n p}^{(j)}\right\rangle \frac{i 8 \Delta \beta-\Delta b_{l m n p}}{\Delta b_{l m n p}^{2}+(8 \Delta \beta)^{2}} \delta^{K}\left(\Delta \omega_{l m n p}\right) .
$$

Proceeding as in section III, we obtain the discrete kinetic equation

$$
\begin{aligned}
\partial_{z} n_{p}(z)= & \frac{4 \gamma^{2} \overline{\Delta \beta}}{3} \sum_{l, m, n} \frac{\delta^{K}\left(\Delta \omega_{l m n p}\right)}{\Delta b_{l m n p}^{2}+\overline{\Delta \beta}^{2}}\left|S_{l m n p}\right|^{2} M_{l m n p}(\boldsymbol{n}) \\
& +\frac{32 \gamma^{2} \overline{\Delta \beta}}{9} \sum_{l} \frac{\delta^{K}\left(\Delta \omega_{l p}\right)}{\Delta b_{l p}^{2}+\overline{\Delta \beta}^{2}}\left|s_{l p}(\boldsymbol{n})\right|^{2}\left(n_{l}-n_{p}\right)(44)
\end{aligned}
$$

where we recall that $s_{l p}(\boldsymbol{n})=\sum_{m^{\prime}} S_{l m^{\prime} m^{\prime} p} n_{m^{\prime}}$, $M_{l m n p}(\boldsymbol{n})=n_{l} n_{m} n_{p}+n_{l} n_{m} n_{n}-n_{n} n_{p} n_{m}-n_{n} n_{p} n_{l}$, with $\Delta b_{l p}=b_{l}-b_{p}$ and $\overline{\Delta \beta}=8 \Delta \beta$. As already commented through Eq.(22), the Lorentzian distribution reflects the finite bandwidth of the four-wave resonances due to the effective dissipation $\Delta \beta$. Accordingly, the kinetic Eq.(44) conserves $E=\sum_{p} \beta_{p} n_{p}(z)$, but not 
$\tilde{E}=\sum_{p} \tilde{\beta}_{p} n_{p}(z)$. In addition, the conservation of the power $N=\sum_{p} n_{p}(z)$ and the $H$-theorem of entropy growth for $\mathcal{S}(z)=\sum_{p} \log \left(n_{p}(z)\right)$ describe a relaxation to $n_{p}^{e q}=T /\left(\beta_{p}-\mu\right)$, i.e., the same equilibrium as in the absence of the correction on the dispersion relation $\left(b_{p}=0\right)$. The main difference is that in the regime $b_{0} \sim \Delta \beta$, the correction $b_{p}$ leads to a deceleration of the rate of thermalization and condensation. On the other hand, in the regime $\Delta \beta \gg b_{0}$, the Lorentzian distribution can be simplified $\overline{\Delta \beta} /\left(\Delta b_{\text {lmnp }}^{2}+\overline{\Delta \beta}^{2}\right) \rightarrow 1 / \overline{\Delta \beta}$, and the kinetic Eq.(44) exactly recovers the previous kinetic Eq.(29), i.e. the correction of the dispersion relation $b_{p}$ does not affect the rate of thermalization.

Let us now discuss the impact of a correction on the dispersion relation in the absence of disorder. This situation is relevant for direct numerical simulations of the (continuous) NLS Eq.(1), where the truncation of the parabolic potential $V(\boldsymbol{r})$ introduces a perturbation on the dispersion relation for higher-order modes (see Fig. 5 in Ref.[17]). At variance with simulations of the (discretized) modal NLS Eq.(4) without disorder that do not evidence wave condensation even for large propagation lengths (see Fig. 1 and the thermalization length scale $L_{k i n}^{\text {ord }} \sim \beta_{0} L_{n l}^{2} / \bar{S}_{\text {lmnp }}^{2}$ ), the simulations of the NLS Eq.(1) show a rather rapid condensation process in the presence of an initial condition with random phases among the modes, as illustrated in Fig. 11. This rapid condensation can be explained by the perturbation on the dispersion relation due to the truncation of the parabolic potential, which modifies the regularization of wave resonances by removing the mode degeneracies, $L_{k i n}^{\text {pert }} \sim b_{p} L_{n l}^{2} / \bar{S}_{l m n p}^{2}$, see Appendix B. However, at variance with experiments of beam cleaning, this estimation of $L_{k i n}^{\text {pert }}$ assumes that the initial condition exhibits random phases among the modes, a feature of fundamental importance for the applicability of the wave turbulence theory $[7,86,87]$. In addition, this estimation assumes that the perturbation $b_{p}$ is maintained fixed, while in a real experiment such a perturbation may not be controlled all over the fiber length due to inherent imperfections and external perturbations, and the corresponding fluctuations contribute to the disorder term in the NLS equation, as discussed through the kinetic Eq.(44) here above.

We finally notice that the refractive index profile of the MMF can be tailored to optimize mode properties. For instance, one can generalize the GRIN parabolic profile by using exponents strongly deviating from 2 , the higher that exponent, the closer the profile would be to a stepindex profile. Since the effect of beam cleaning does not occur in step-index fibers (see section VI A), it would be interesting to study the degradation of wave condensation in the discrete turbulence regime by gradually increasing the exponent of the refractive index profile, in line with the experiments initiated in [94].
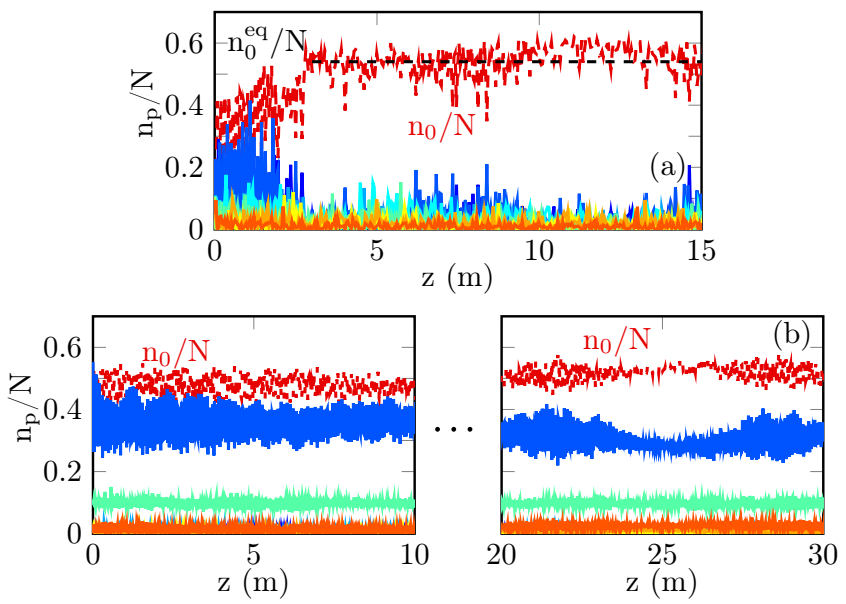

FIG. 11: Numerical simulations of the 'continuous' NLS Eq.(1) showing the evolutions of the modal populations $n_{p} / N$ : fundamental mode $p=0$ (red dashed), $p=1$

(dark blue solid), $p=2$ (blue solid), $p=3$ (light blue solid), $p=4$ (cyan solid), $p=5$ (light green solid), $p=6$ (green solid), $p=7$ (yellow solid), $p=8$ (orange solid). (a) Starting from a 'speckle' beam with random phase among the modes, the beam exhibits a condensation process: $n_{0}(z) / N$ relaxes to the theoretical equilibrium value $n_{0}^{e q} / N \simeq 0.54$ (dashed

black line from Eq.(31)). (b) Starting from a coherent initial condition, the modal components exhibit an oscillatory behavior, similar to that observed for the

discretized modal NLS Eq.(4), see Fig. 1(a). At complete thermal equilibrium, the fundamental mode would reach the condensate fraction $n_{0}^{e q} / N \simeq 0.8$. The power is $N=47.5 \mathrm{~kW}\left(N_{*}=66\right.$ modes, $\left.a=15 \mu \mathrm{m}\right)$.

\section{CONCLUSION}

In summary, we have discussed experiments of beam self-cleaning where long $(\sim n s)$ pulses are injected in relative short multimode fiber lengths $(\sim 10 \mathrm{~m})$, for which the dominant contribution of disorder originates from polarization random fluctuations (weak disorder) [45]. On the basis of the wave turbulence theory, we have derived kinetic equations describing the nonequilibrium evolution of random waves in a regime where disorder dominates nonlinear effects $\left(L_{d} \ll L_{n l}\right)$. The theory revealed that the presence of a conservative weak disorder introduces an effective dissipation in the system whose resonance broadening prevents the conservation of the energy, which inhibits the effect of condensation in the usual continuous wave turbulence approach. On the other hand, we have shown that usual experiments of beamcleaning are not described by the continuous wave turbulence theory, but instead by a discrete wave turbulence approach. In this discrete turbulence regime only exact resonances contribute to the kinetic equation, which is no longer sensitive to the effect of dissipation-induced 
resonance broadening. Accordingly, the discrete kinetic equation conserves the energy, which re-establishes the process of wave condensation. The main result is that the effective dissipation induced by disorder modifies the regularization of such discrete resonances, which leads to an acceleration of the rate of thermalization and condensation.

In order to improve our understanding of beam cleaning experiments, we have considered different models of weak disorder in MMFs. The theory shows that when all modes experience the same (mode-correlated) noise, the dissipation induced by disorder vanishes and the system no longer exhibits a fast process of condensation. However, even a relative small decorrelation among the noise experienced by the modes is sufficient to re-establish a disorder-induced acceleration of condensation. The simulations are in quantitative agreement with the theory without using adjustable parameters in the regime where disorder dominates nonlinear effects $\left(L_{d} \ll L_{n l}\right)$. However, the impact of weak disorder due to polarization fluctuations in beam cleaning experiments is expected to be of the same order as nonlinear effects [45]. We have thus considered the impact of a moderate disorder $\left(L_{d} \gtrsim L_{n l}\right)$. In this regime, the phase-correlations among the modes are not completely suppressed and the system enters a mixed coherent-incoherent regime. Accordingly, the modal components exhibit an oscillatory behavior that slows down the thermalization process, though the optical beam still exhibits a fast condensation process that is consistent with the experimental results reported in Ref.[41]. In addition, the analysis of polarization effects revealed that optical beam cleaning is responsible for an effective partial repolarization of the central part of the beam. This property was observed experimentally in Ref.[54] and it can be interpreted as a natural consequence of the condensation-induced macroscopic population of the fundamental mode of the MMF. To summarize, by considering the dominant contribution of weak disorder originating in polarization fluctuations, our theory and simulations provide a qualitative understanding of the effect of optical beam self-cleaning, in particular when a large number of modes are excited into the MMF as in the recent experiments reported in Ref.[41]. On the other hand, the discrete nature of wave turbulence in MMFs is responsible for a freezing of thermalization and condensation when a small number of modes are excited. Such an effective freezing of condensation also explains why optical beam cleaning has not been observed in stepindex MMFs.

It is important to recall that, at variance with the experiments of beam cleaning where sub-nanosecond pulsed are injected into the MMF, we considered in this work a purely spatial NLS equation to model the propagation of the beam through the fiber. This is justified by the fact that in the nanosecond regime temporal dispersion effects can be neglected in first approximation. Then although our theory and simulations describe the essential mechanism underlying beam cleaning condensation, it is clear that they cannot provide a quantitative description of the experimental results, which would require a detailed analysis of the temporal averaging effect inherent to the pulsed regime considered in the experimental measurements.

We have also considered the impact of strong disorder at the leading order linear regime, which revealed that random mode coupling among degenerate modes enforces the thermalization toward the Rayleigh-Jeans equilibrium distribution. We note in this respect that, according to the simulations reported in [42], strong disorder only weakly affects the evolutions of the modal components as compared to the significant impact of weak disorder discussed in this work. Nevertheless, it would be important to extend the theory developed for weak disorder to strong disorder in order to study the interplay of random mode coupling and nonlinearity, a feature that will be considered in future works.

From a broader perspective, the wave turbulence theory discussed in this work can be extended to study spatio-temporal effects in MMFs [37, 38, 68-72, 95-98]. The development of a spatio-temporal theory would also be important to study complex incoherent behaviors, such as the formation of incoherent shocks [99, 100], or the generation of supercontinuum radiation in MMFs $[72,101-103]$, in relation with the wave turbulence kinetic approach developed to study supercontinuum generation in single-mode fibers [104-106]. The discrete wave turbulence approach discussed in this work can also be extended to study the impact of disorder on turbulence cascades [7, 8, 15, 107], from both the theoretical and experimental points of views. Extension of the theory to consider other forms of nonlinear effects would also be interesting, such as saturable or nonlocal nonlinearities in relation with atomic vapors beam cleaning experiments [108]. In addition, there is a growing interest for experimental demonstrations of superfluid light flows in bulk materials [109-111]. The experiments of condensation in MMFs would allow to study the nucleation of superfluid vortices induced by a rotating confining potential (along the 'time' $z$-variable) in manufactured multimode fibers, in analogy with rotating trapped BECs [1].

The acceleration of the process of thermalization reported in this work is also relevant to the notion of prethermalization to out of equilibrium states $[23,112-$ 115], which is attracting a growing interest in different research communities, including long range interacting systems with fast relaxation towards quasi-stationary states [116-118], or one dimensional (nearly) integrable (quantum) systems [119-123]. From a broader perspective, the present work can contribute to the challenging question of spontaneous organization of coherent states in nonlinear disordered (turbulent) systems [84, 85, 124$128]$, in relation with the paradigm of statistical lightmode dynamics (glassy behaviors) and complexity in random lasers [129, 130]. 


\section{ACKNOWLEDGEMENTS}

The authors are grateful to A. Tonello, P. Béjot, S. Guérin, V. Couderc, and A. Barthélemy for fruitful discussions. We acknowledge financial support from the French ANR under Grant No. ANR-19-CE460007 (project ICCI), iXcore research foundation, EIPHI Graduate School (Contract No. ANR-17-EURE-0002),
French program "Investissement d'Avenir," Project No. ISITE-BFC-299 (ANR-15 IDEX-0003); H2020 Marie Sklodowska-Curie Actions (MSCA-COFUND) (MULTIPLY Project No. 713694). Calculations were performed using HPC resources from DNUM CCUB (Centre de Calcul, Université de Bourgogne).

\section{APPENDIX A: DERIVATION OF THE DISCRETE KINETIC EQUATION WITH WEAK DISORDER}

To study correlations among the modes, we derive an equation for the moments of the $2 \times 2$ matrix $\left\langle\boldsymbol{A}_{p}^{*} \boldsymbol{A}_{q}^{T}\right\rangle(z)$. The computation for distinct modes $(p \neq q)$ was reported in the Supplemental of Ref.[41]. The computation for the correlations within a specific mode $(p=q)$ is more delicate and it is detailed here below.

\section{A. Modal correlations}

The second moments satisfy:

$$
\partial_{z}\left\langle\boldsymbol{A}_{p}^{*} \boldsymbol{A}_{p}^{T}\right\rangle=i\left\langle\mathbf{D}_{p}^{*} \boldsymbol{A}_{p}^{*} \boldsymbol{A}_{p}^{T}\right\rangle-i\left\langle\boldsymbol{A}_{p}^{*} \boldsymbol{A}_{p}^{T} \mathbf{D}_{p}^{T}\right\rangle-i \gamma\left\langle\boldsymbol{G}_{p p}(\boldsymbol{A})\right\rangle .
$$

where $\boldsymbol{G}_{p q}(\boldsymbol{A}(z))=\boldsymbol{P}_{p}(\boldsymbol{A})^{*} \boldsymbol{A}_{q}^{T}(z)-\boldsymbol{A}_{p}^{*} \boldsymbol{P}_{q}(\boldsymbol{A})^{T}(z)$. According to the Furutsu-Novikov theorem:

$$
\left\langle\nu_{p, j} \boldsymbol{\sigma}_{j}^{*} \boldsymbol{A}_{p}^{*} \boldsymbol{A}_{p}^{T}\right\rangle=\int_{0}^{z}\left\langle\frac{\delta\left(\boldsymbol{\sigma}_{j}^{*} \boldsymbol{A}_{p}^{*} \boldsymbol{A}_{p}^{T}(z)\right)}{\delta \nu_{p, j}\left(z^{\prime}\right)}\right\rangle \sigma_{\beta}^{2} \mathcal{R}\left(\frac{z-z^{\prime}}{l_{\beta}}\right) d z^{\prime}
$$

The variational derivative can be computed by following [131]. For $z>z^{\prime}$ it is the solution of

$$
\partial_{z} \frac{\delta\left(\boldsymbol{A}_{p}^{*} \boldsymbol{A}_{p}^{T}(z)\right)}{\delta \nu_{p, j}\left(z^{\prime}\right)}=i \mathbf{D}_{p}^{*} \frac{\delta\left(\boldsymbol{A}_{p}^{*} \boldsymbol{A}_{p}^{T}(z)\right)}{\delta \nu_{p, j}\left(z^{\prime}\right)}-i \frac{\delta\left(\boldsymbol{A}_{p}^{*} \boldsymbol{A}_{p}^{T}(z)\right)}{\delta \nu_{p, j}\left(z^{\prime}\right)} \mathbf{D}_{p}^{T}-i \gamma \frac{\delta\left(\boldsymbol{G}_{p p}(\boldsymbol{A}(z))\right)}{\delta \nu_{p, j}\left(z^{\prime}\right)}
$$

starting from

$$
\left.\frac{\delta\left(\boldsymbol{A}_{p}^{*} \boldsymbol{A}_{p}^{T}(z)\right)}{\delta \nu_{p, j}\left(z^{\prime}\right)}\right|_{z=z^{\prime}}=i \boldsymbol{\sigma}_{j}^{*} \boldsymbol{A}_{p}^{*} \boldsymbol{A}_{p}^{T}(z)-i \boldsymbol{A}_{p}^{*} \boldsymbol{A}_{p}^{T}(z) \boldsymbol{\sigma}_{j}^{T}
$$

We need to know the form of the variational derivative for $z^{\prime}<z$ and $\left|z-z^{\prime}\right|=O\left(l_{\beta}\right)$. As $\sigma_{\beta} l_{\beta} \ll 1$ and $l_{\beta} \ll L_{n l}$, all terms in the right-hand side of the differential equation (45) satisfied by the variational derivative are negligeable for $\left|z-z^{\prime}\right|=O\left(l_{\beta}\right)$, so that the leading-order expression of the variational derivative for $z^{\prime}<z$ and $\left|z-z^{\prime}\right|=O\left(l_{\beta}\right)$ is

$$
\frac{\delta\left(\boldsymbol{A}_{p}^{*} \boldsymbol{A}_{p}^{T}(z)\right)}{\delta \nu_{p, j}\left(z^{\prime}\right)}=i \boldsymbol{\sigma}_{j}^{*} \boldsymbol{A}_{p}^{*} \boldsymbol{A}_{p}^{T}\left(z^{\prime}\right)-i \boldsymbol{A}_{p}^{*} \boldsymbol{A}_{p}^{T}\left(z^{\prime}\right) \boldsymbol{\sigma}_{j}^{T},
$$

and therefore

$$
\left\langle\nu_{p, j} \boldsymbol{\sigma}_{j}^{*} \boldsymbol{A}_{p}^{*} \boldsymbol{A}_{p}^{T}\right\rangle=i \int_{0}^{z}\left\langle\boldsymbol{\sigma}_{j}^{*} \boldsymbol{\sigma}_{j}^{*} \boldsymbol{A}_{p}^{*} \boldsymbol{A}_{p}^{T}\left(z^{\prime}\right)\right\rangle \sigma_{\beta}^{2} \mathcal{R}\left(\frac{z-z^{\prime}}{l_{\beta}}\right) d z^{\prime}-i \int_{0}^{z}\left\langle\boldsymbol{\sigma}_{j}^{*} \boldsymbol{A}_{p}^{*} \boldsymbol{A}_{p}^{T}\left(z^{\prime}\right) \boldsymbol{\sigma}_{j}^{T}\right\rangle \sigma_{\beta}^{2} \mathcal{R}\left(\frac{z-z^{\prime}}{l_{\beta}}\right) d z^{\prime}
$$

For $j=0$ this is zero and for $j=1,2,3$ this can be approximated by (using $l_{\beta} \ll z$ ):

$$
\left\langle\nu_{p, j} \boldsymbol{\sigma}_{j}^{*} \boldsymbol{A}_{p}^{*} \boldsymbol{A}_{p}^{T}\right\rangle=\frac{i \Delta \beta}{2}\left(\left\langle\boldsymbol{\sigma}_{j}^{*} \boldsymbol{\sigma}_{j}^{*} \boldsymbol{A}_{p}^{*} \boldsymbol{A}_{p}^{T}\right\rangle-\left\langle\boldsymbol{\sigma}_{j}^{*} \boldsymbol{A}_{p}^{*} \boldsymbol{A}_{p}^{T} \boldsymbol{\sigma}_{j}^{T}\right\rangle\right),
$$

and we find

$$
\partial_{z}\left\langle\boldsymbol{A}_{p}^{*} \boldsymbol{A}_{p}^{T}\right\rangle=-\Delta \beta\left(3\left\langle\boldsymbol{A}_{p}^{*} \boldsymbol{A}_{p}^{T}\right\rangle-\sum_{j=1}^{3} \boldsymbol{\sigma}_{j}^{*}\left\langle\boldsymbol{A}_{p}^{*} \boldsymbol{A}_{p}^{T}\right\rangle \boldsymbol{\sigma}_{j}^{T}\right)-i \gamma\left\langle\boldsymbol{G}_{p p}(\boldsymbol{A})\right\rangle
$$


The mean $2 \times 2$ matrix $\left\langle\boldsymbol{A}_{p}^{*} \boldsymbol{A}_{p}^{T}\right\rangle$ is Hermitian and therefore it can be expanded as

$$
\left\langle\boldsymbol{A}_{p}^{*} \boldsymbol{A}_{p}^{T}\right\rangle=w_{p}(z) \boldsymbol{\sigma}_{0}+\sum_{j=1}^{3} w_{p, j}(z) \boldsymbol{\sigma}_{j}
$$

The real-valued functions $w_{p}$ and $\left(w_{p, j}\right)_{j=1}^{3}$ satisfy

$$
\begin{aligned}
\partial_{z} w_{p} & =-\gamma \boldsymbol{\Pi}_{0}\left\{\left\langle i \boldsymbol{P}_{p}(\boldsymbol{A})^{*} \boldsymbol{A}_{p}^{T}-i \boldsymbol{A}_{p}^{*} \boldsymbol{P}_{p}(\boldsymbol{A})^{T}\right\rangle\right\}, \\
\partial_{z} w_{p, j} & =-4 \Delta \beta w_{p, j}-\gamma \boldsymbol{\Pi}_{j}\left\{\left\langle i \boldsymbol{G}_{p p}(\boldsymbol{A})\right\rangle\right\}
\end{aligned}
$$

where $\boldsymbol{\Pi}_{j} \mathbf{W}=$ coefficient of the decomposition of the Hermitian matrix $\mathbf{W}$ on $\boldsymbol{\sigma}_{j}$. In particular, $\boldsymbol{\Pi}_{0} \mathbf{W}=\operatorname{Tr}(\mathbf{W}) / 2$ so that

$$
\boldsymbol{\Pi}_{0}\left\{\left\langle i \boldsymbol{G}_{p p}(\boldsymbol{A})\right\rangle\right\}=-\operatorname{Im}\left\langle\boldsymbol{P}_{p}(\boldsymbol{A})^{\dagger} \boldsymbol{A}_{p}\right\rangle .
$$

The coefficients $w_{p, j}$ for $j=1,2,3$ satisfy the damped equations

$$
\partial_{z} w_{p, j}=-4 \Delta \beta w_{p, j}-\gamma \boldsymbol{\Pi}_{j}\left\{\left\langle i \boldsymbol{G}_{p p}(\boldsymbol{A})\right\rangle\right\}
$$

They are of the form

$$
w_{p, j}(z)=w_{p, j}(0) \exp (-4 \Delta \beta z)-\gamma \int_{0}^{z} \exp \left(-4 \Delta \beta\left(z-z^{\prime}\right)\right) \boldsymbol{\Pi}_{j}\left\{\left\langle i \boldsymbol{G}_{p p}(\boldsymbol{A})\right\rangle\right\}\left(z^{\prime}\right) d z^{\prime}
$$

As we have $z, L_{n l} \gg 1 / \Delta \beta$, the initial condition is forgotten in (48) and the second term in the right-hand side can be simplified and we get for $j=1,2,3$

$$
w_{p, j}=-\frac{\gamma}{4 \Delta \beta} \boldsymbol{\Pi}_{j}\left\{\left\langle i \boldsymbol{G}_{p p}(\boldsymbol{A}(z))\right\rangle\right\}
$$

Using the assumption $L_{d}=1 / \Delta \beta \ll L_{n l}$, we have

$$
w_{p, j} \simeq 0
$$

to leading order.

\section{B. Closure of the moments equations}

The diagonal modal components $w_{p}$ in (46) satisfy the undamped equation

$$
\partial_{z} w_{p}=\gamma \operatorname{Im}\left\langle\boldsymbol{P}_{p}(\boldsymbol{A})^{\dagger} \boldsymbol{A}_{p}\right\rangle
$$

With the expression (5) of the nonlinear term, we get that the mode occupancies $w_{p}$ satisfy the coupled equations (13-15). We now derive the equations governing the evolutions of the fourth-order moments $\left\langle X_{p}^{(1)}\right\rangle$ and $\left\langle X_{p}^{(2)}\right\rangle$ given by Eqs.(14-15). 
1. Computation of the term $\left\langle X_{p}^{(1)}\right\rangle$ :

We first write the equation satisfied by the product of four vector fields:

$$
\begin{aligned}
\partial_{z}\left(\boldsymbol{A}_{l}^{\dagger} \boldsymbol{A}_{m}^{*}\right)\left(\boldsymbol{A}_{n}^{T} \boldsymbol{A}_{p}\right)= & i\left(\beta_{l}+\beta_{m}-\beta_{n}-\beta_{p}\right)\left(\boldsymbol{A}_{l}^{\dagger} \boldsymbol{A}_{m}^{*}\right)\left(\boldsymbol{A}_{n}^{T} \boldsymbol{A}_{p}\right)+i\left(\boldsymbol{A}_{l}^{\dagger} \mathbf{D}_{l}^{\dagger} \boldsymbol{A}_{m}^{*}\right)\left(\boldsymbol{A}_{n}^{T} \boldsymbol{A}_{p}\right) \\
& +i\left(\boldsymbol{A}_{l}^{\dagger} \mathbf{D}_{m}^{*} \boldsymbol{A}_{m}^{*}\right)\left(\boldsymbol{A}_{n}^{T} \boldsymbol{A}_{p}\right)-i\left(\boldsymbol{A}_{l}^{\dagger} \boldsymbol{A}_{m}^{*}\right)\left(\boldsymbol{A}_{n}^{T} \mathbf{D}_{n}^{T} \boldsymbol{A}_{p}\right)-i\left(\boldsymbol{A}_{l}^{\dagger} \boldsymbol{A}_{m}^{*}\right)\left(\boldsymbol{A}_{n}^{T} \mathbf{D}_{p} \boldsymbol{A}_{p}\right) \\
& +i \gamma Y_{l m n p}^{(1)}, \\
Y_{l m n p}^{(1)}= & -\sum_{l^{\prime}, m^{\prime}, n^{\prime}} S_{l^{\prime} m^{\prime} n^{\prime} l}^{*}\left[\frac{1}{3}\left(\boldsymbol{A}_{l^{\prime}}^{\dagger} \boldsymbol{A}_{m^{\prime}}^{*}\right)\left(\boldsymbol{A}_{n^{\prime}}^{T} \boldsymbol{A}_{m}^{*}\right)+\frac{2}{3}\left(\boldsymbol{A}_{n^{\prime}}^{T} \boldsymbol{A}_{m^{\prime}}^{*}\right)\left(\boldsymbol{A}_{l^{\prime}}^{\dagger} \boldsymbol{A}_{m}^{*}\right)\right]\left(\boldsymbol{A}_{n}^{T} \boldsymbol{A}_{p}\right) \\
& -\sum_{l^{\prime}, m^{\prime}, n^{\prime}} S_{l^{\prime} m^{\prime} n^{\prime} m}^{*}\left[\frac{1}{3}\left(\boldsymbol{A}_{l^{\prime}}^{\dagger} \boldsymbol{A}_{m^{\prime}}^{*}\right)\left(\boldsymbol{A}_{l}^{\dagger} \boldsymbol{A}_{n^{\prime}}\right)+\frac{2}{3}\left(\boldsymbol{A}_{n^{\prime}}^{T} \boldsymbol{A}_{m^{\prime}}^{*}\right)\left(\boldsymbol{A}_{l}^{\dagger} \boldsymbol{A}_{l^{\prime}}^{*}\right)\right]\left(\boldsymbol{A}_{n}^{T} \boldsymbol{A}_{p}\right) \\
& +\sum_{l^{\prime}, m^{\prime}, n^{\prime}} S_{l^{\prime} m^{\prime} n^{\prime} n}\left[\frac{1}{3}\left(\boldsymbol{A}_{l^{\prime}}^{T} \boldsymbol{A}_{m^{\prime}}\right)\left(\boldsymbol{A}_{n^{\prime}}^{\dagger} \boldsymbol{A}_{p}\right)+\frac{2}{3}\left(\left(\boldsymbol{A}_{n^{\prime}}^{\dagger} \boldsymbol{A}_{m^{\prime}}\right)\left(\boldsymbol{A}_{l^{\prime}}^{T} \boldsymbol{A}_{p}\right)\right]\left(\boldsymbol{A}_{l}^{\dagger} \boldsymbol{A}_{m}^{*}\right)\right. \\
& +\sum_{l^{\prime}, m^{\prime}, n^{\prime}} S_{l^{\prime} m^{\prime} n^{\prime} p}\left[\frac{1}{3}\left(\boldsymbol{A}_{l^{\prime}}^{T} \boldsymbol{A}_{m^{\prime}}\right)\left(\boldsymbol{A}_{n}^{T} \boldsymbol{A}_{n^{\prime}}^{*}\right)+\frac{2}{3}\left(\boldsymbol{A}_{n^{\prime}}^{\dagger} \boldsymbol{A}_{m^{\prime}}\right)\left(\boldsymbol{A}_{n}^{T} \boldsymbol{A}_{l^{\prime}}\right)\right]\left(\boldsymbol{A}_{l}^{\dagger} \boldsymbol{A}_{m}^{*}\right)
\end{aligned}
$$

We take the expectation and we apply the Gaussian summation rule to the sixth-order moments in the expression of $\left\langle Y_{l m n p}^{(1)}\right\rangle:$

$$
\begin{aligned}
\left\langle Y_{l m n p}^{(1)}\right\rangle= & \frac{16}{3} S_{l m n p}\left(w_{l} w_{m} w_{p}+w_{l} w_{m} w_{n}-w_{n} w_{p} w_{m}-w_{n} w_{p} w_{l}\right)+\frac{16}{3} \delta_{m p}^{K} s_{l n}(\boldsymbol{w}) w_{p}\left(w_{l}-w_{n}\right) \\
& +\frac{16}{3} \delta_{m n}^{K} s_{l p}(\boldsymbol{w}) w_{n}\left(w_{l}-w_{p}\right)+\frac{16}{3} \delta_{l p}^{K} s_{m n}(\boldsymbol{w}) w_{p}\left(w_{m}-w_{n}\right)+\frac{16}{3} \delta_{l n}^{K} s_{m p}(\boldsymbol{w}) w_{n}\left(w_{m}-w_{p}\right) \\
s_{l n}(\boldsymbol{w})= & \sum_{n^{\prime}} S_{l n^{\prime} n^{\prime} n} w_{n^{\prime}}
\end{aligned}
$$

We now extend the procedure of Sec. IX A to the computation of fourth-order modes considered here. Making use of the Furutsu-Novikov theorem, and considering the general case $l \neq m \neq n \neq p$ in the regime $\sigma_{\beta} l_{\beta} \ll 1$ and $l_{\beta} \ll L_{n l}$, we obtain

$$
\partial_{z}\left\langle\left(\boldsymbol{A}_{l}^{\dagger} \boldsymbol{A}_{m}^{*}\right)\left(\boldsymbol{A}_{n}^{T} \boldsymbol{A}_{p}\right)\right\rangle=\left(-8 \Delta \beta+i\left(\beta_{l}+\beta_{m}-\beta_{n}-\beta_{p}\right)\right)\left\langle\left(\boldsymbol{A}_{l}^{\dagger} \boldsymbol{A}_{m}^{*}\right)\left(\boldsymbol{A}_{n}^{T} \boldsymbol{A}_{p}\right)\right\rangle+i \gamma\left\langle Y_{l m n p}^{(1)}\right\rangle
$$

whose solution has the form

$$
\begin{aligned}
\left\langle\left(\boldsymbol{A}_{l}^{\dagger} \boldsymbol{A}_{m}^{*}\right)\left(\boldsymbol{A}_{n}^{T} \boldsymbol{A}_{p}\right)\right\rangle(z)= & \left\langle\left(\boldsymbol{A}_{l}^{\dagger} \boldsymbol{A}_{m}^{*}\right)\left(\boldsymbol{A}_{n}^{T} \boldsymbol{A}_{p}\right)\right\rangle(0) \exp \left(\left(-8 \Delta \beta+i\left(\beta_{l}+\beta_{m}-\beta_{n}-\beta_{p}\right)\right) z\right) \\
& +i \gamma \int_{0}^{z}\left\langle Y_{l m n p}^{(1)}\right\rangle\left(z^{\prime}\right) \exp \left(\left(-8 \Delta \beta+i\left(\beta_{l}+\beta_{m}-\beta_{n}-\beta_{p}\right)\right)\left(z-z^{\prime}\right)\right) d z^{\prime}
\end{aligned}
$$

These equations correspond to those reported in Eq.(16) and Eq.(17) for the fourth-order moment $J_{l m n p}^{(1)}$.

In the other cases when (at least) two indices are equal, i.e., the fourth-order moments involve degenerate modes, the calculation shows that there is still a damping in the moment equation, although the damping factor can be different from $8 \Delta \beta$. However we will neglect this change because: (i) these terms are negligible in the triple sum and when the number of modes is large $\left(N_{*} \gg 1\right)$, and (ii) this change only affects the multiplicative coefficient $8 \Delta \beta$ in (54). Finally we give the expression of $\left\langle X_{p}^{(1)}\right\rangle$ in the discrete wave turbulence regime where $\beta_{0} \gg \Delta \beta$. Collecting all terms we obtain

$$
\begin{aligned}
\left\langle X_{p}^{(1)}\right\rangle= & \frac{2 \gamma}{3 \Delta \beta} \sum_{l, m, n}\left|S_{l m n p}\right|^{2} \delta^{K}\left(\beta_{l}+\beta_{m}-\beta_{n}-\beta_{p}\right)\left(w_{l} w_{m} w_{p}+w_{l} w_{m} w_{n}-w_{n} w_{p} w_{m}-w_{n} w_{p} w_{l}\right) \\
& +\frac{4 \gamma}{3 \Delta \beta} \sum_{l}\left|s_{l p}(\boldsymbol{w})\right|^{2} \delta^{K}\left(\beta_{l}-\beta_{p}\right)\left(w_{l}-w_{p}\right) .
\end{aligned}
$$


2. Computation of the term $\left\langle X_{p}^{(2)}\right\rangle$ :

The equation satisfied by the product of four vector fields reads

$$
\begin{aligned}
\partial_{z}\left(\boldsymbol{A}_{n}^{T} \boldsymbol{A}_{m}^{*}\right)\left(\boldsymbol{A}_{l}^{\dagger} \boldsymbol{A}_{p}\right)= & i\left(\beta_{l}+\beta_{m}-\beta_{n}-\beta_{p}\right)\left(\boldsymbol{A}_{n}^{T} \boldsymbol{A}_{m}^{*}\right)\left(\boldsymbol{A}_{l}^{\dagger} \boldsymbol{A}_{p}\right)+i\left(\boldsymbol{A}_{n}^{T} \boldsymbol{A}_{m}^{*}\right)\left(\boldsymbol{A}_{l}^{\dagger} \mathbf{D}_{l}^{\dagger} \boldsymbol{A}_{p}\right) \\
& +i\left(\boldsymbol{A}_{n}^{T} \mathbf{D}_{m}^{*} \boldsymbol{A}_{m}^{*}\right)\left(\boldsymbol{A}_{l}^{\dagger} \boldsymbol{A}_{p}\right)-i\left(\boldsymbol{A}_{n}^{T} \mathbf{D}_{n}^{T} \boldsymbol{A}_{m}^{*}\right)\left(\boldsymbol{A}_{l}^{\dagger} \boldsymbol{A}_{p}\right)-i\left(\boldsymbol{A}_{n}^{T} \boldsymbol{A}_{m}^{*}\right)\left(\boldsymbol{A}_{l}^{\dagger} \mathbf{D}_{p} \boldsymbol{A}_{p}\right) \\
& +i \gamma Y_{l m n p}^{(2)}, \\
Y_{l m n p}^{(2)}= & -\sum_{l^{\prime}, m^{\prime}, n^{\prime}} S_{l^{\prime} m^{\prime} n^{\prime} l}^{*}\left[\frac{1}{3}\left(\boldsymbol{A}_{l^{\prime}}^{\dagger} \boldsymbol{A}_{m^{\prime}}^{*}\right)\left(\boldsymbol{A}_{n^{\prime}}^{T} \boldsymbol{A}_{p}\right)+\frac{2}{3}\left(\boldsymbol{A}_{n^{\prime}}^{T} \boldsymbol{A}_{m^{\prime}}^{*}\right)\left(\boldsymbol{A}_{l^{\prime}}^{\dagger} \boldsymbol{A}_{p}\right)\right]\left(\boldsymbol{A}_{m}^{\dagger} \boldsymbol{A}_{n}\right) \\
& -\sum_{l^{\prime}, m^{\prime}, n^{\prime}} S_{l^{\prime} m^{\prime} n^{\prime} m}^{*}\left[\frac{1}{3}\left(\boldsymbol{A}_{l^{\prime}}^{\dagger} \boldsymbol{A}_{m^{\prime}}^{*}\right)\left(\boldsymbol{A}_{n^{\prime}}^{T} \boldsymbol{A}_{n}\right)+\frac{2}{3}\left(\boldsymbol{A}_{n^{\prime}}^{T} \boldsymbol{A}_{m^{\prime}}^{*}\right)\left(\boldsymbol{A}_{l^{\prime}}^{\dagger} \boldsymbol{A}_{n}\right)\right]\left(\boldsymbol{A}_{l}^{\dagger} \boldsymbol{A}_{p}\right) \\
& +\sum_{l^{\prime}, m^{\prime}, n^{\prime}} S_{l^{\prime} m^{\prime} n^{\prime} n}\left[\frac{1}{3}\left(\boldsymbol{A}_{l^{\prime}}^{T} \boldsymbol{A}_{m^{\prime}}\right)\left(\boldsymbol{A}_{n^{\prime}}^{\dagger} \boldsymbol{A}_{m}^{*}\right)+\frac{2}{3}\left(\boldsymbol{A}_{n^{\prime}}^{\dagger} \boldsymbol{A}_{m^{\prime}}\right)\left(\boldsymbol{A}_{l^{\prime}}^{T} \boldsymbol{A}_{m}^{*}\right)\right]\left(\boldsymbol{A}_{l}^{\dagger} \boldsymbol{A}_{p}\right) \\
& +\sum_{l^{\prime}, m^{\prime}, n^{\prime}} S_{l^{\prime} m^{\prime} n^{\prime} p}\left[\frac{1}{3}\left(\boldsymbol{A}_{l^{\prime}}^{T} \boldsymbol{A}_{m^{\prime}}\right)\left(\boldsymbol{A}_{n^{\prime}}^{\dagger} \boldsymbol{A}_{l}^{*}\right)+\frac{2}{3}\left(\boldsymbol{A}_{n^{\prime}}^{\dagger} \boldsymbol{A}_{m^{\prime}}\right)\left(\boldsymbol{A}_{l^{\prime}}^{T} \boldsymbol{A}_{l}^{*}\right)\right]\left(\boldsymbol{A}_{m}^{\dagger} \boldsymbol{A}_{n}\right) .
\end{aligned}
$$

We take the expectation and we apply the Gaussian summation rule to the sixth-order moments in the expression of $\left\langle Y_{l m n p}^{(2)}\right\rangle:$

$$
\begin{aligned}
\left\langle Y_{l m n p}^{(2)}\right\rangle= & \frac{16}{3} S_{l m n p}\left(w_{l} w_{m} w_{p}+w_{l} w_{m} w_{n}-w_{n} w_{p} w_{m}-w_{n} w_{p} w_{l}\right)+\frac{16}{3} \delta_{m p}^{K} s_{l n}(\boldsymbol{w}) w_{p}\left(w_{l}-w_{n}\right) \\
& +\frac{32}{3} \delta_{m n}^{K} s_{l p}(\boldsymbol{w}) w_{n}\left(w_{l}-w_{p}\right)+\frac{32}{3} \delta_{l p}^{K} s_{m n}(\boldsymbol{w}) w_{p}\left(w_{m}-w_{n}\right)+\frac{16}{3} \delta_{l n}^{K} s_{m p}(\boldsymbol{w}) w_{n}\left(w_{m}-w_{p}\right)
\end{aligned}
$$

If $l \neq m \neq n \neq p$, then Furutsu-Novikov formula gives

$$
\partial_{z}\left\langle\left(\boldsymbol{A}_{n}^{T} \boldsymbol{A}_{m}^{*}\right)\left(\boldsymbol{A}_{l}^{\dagger} \boldsymbol{A}_{p}\right)\right\rangle=\left(-8 \Delta \beta+i\left(\beta_{l}+\beta_{m}-\beta_{n}-\beta_{p}\right)\right)\left\langle\left(\boldsymbol{A}_{n}^{T} \boldsymbol{A}_{m}^{*}\right)\left(\boldsymbol{A}_{l}^{\dagger} \boldsymbol{A}_{p}\right)\right\rangle+i \gamma\left\langle Y_{l m n p}^{(2)}\right\rangle .
$$

This equation corresponds to that reported in Eq.(16) for the fourth-order moment $J_{l m n p}^{(2)}$.

Following the same procedure, we have also derived the following equation

$$
\partial_{z}\left\langle\left(\boldsymbol{A}_{l}^{T} \boldsymbol{A}_{l}^{*}\right)\left(\boldsymbol{A}_{m}^{\dagger} \boldsymbol{A}_{m}\right)\right\rangle=0
$$

for any $l, m$. This implies in particular that the variance of the intensity fluctuations of each modal component $p$ is preserved $\left\langle\left|\boldsymbol{A}_{p}\right|^{4}\right\rangle=$ const, i.e., Gaussian statistics is preserved during the propagation.

Note that Eq.(59) is undamped, but this does not affect our results. Indeed, $\left\langle\left(\boldsymbol{A}_{l}^{T} \boldsymbol{A}_{l}^{*}\right)\left(\boldsymbol{A}_{m}^{\dagger} \boldsymbol{A}_{m}\right)\right\rangle$ is real-valued so that it does not contribute when it is substituted into Eq.(15) because $S_{l m m l}$ is real-valued as well.

By neglecting the small corrections that appear in the cases when (at least) two indices are equal (i.e. fourth-order modes involving degenerate modes), we obtain in the discrete wave turbulence regime $\left(\beta_{0} \gg \Delta \beta\right)$ :

$$
\begin{aligned}
\left\langle X_{p}^{(2)}\right\rangle= & \frac{2 \gamma}{3 \Delta \beta} \sum_{l, m, n} \delta^{K}\left(\beta_{l}+\beta_{m}-\beta_{n}-\beta_{p}\right)\left|S_{l m n p}\right|^{2}\left(w_{l} w_{m} w_{p}+w_{l} w_{m} w_{n}-w_{n} w_{p} w_{m}-w_{n} w_{p} w_{l}\right) \\
& +\frac{2 \gamma}{\Delta \beta} \sum_{l} \delta^{K}\left(\beta_{l}-\beta_{p}\right)\left|s_{l p}(\boldsymbol{w})\right|^{2}\left(w_{l}-w_{p}\right)
\end{aligned}
$$

By replacing the expressions of $\left\langle X_{p}^{(j)}\right\rangle(j=1,2)$ given in (56) and (60) into the equation for the evolution of the modal components (13), we obtain the discrete kinetic Eq.(29). 


\section{Impact of a correlated noise model of disorder on the kinetic equation}

1. Computation of the moment $\left\langle\left(\boldsymbol{A}_{l}^{\dagger} \boldsymbol{A}_{m}^{*}\right)\left(\boldsymbol{A}_{n}^{T} \boldsymbol{A}_{p}\right)\right\rangle$

In the model of correlated disorder, all modes experience the same noise, $\mathbf{D}_{n} \equiv \mathbf{D}=\sum_{j=0}^{3} \nu_{j} \boldsymbol{\sigma}_{j}$. The equation (51) for the evolution of the product of four fields now reads

$$
\begin{aligned}
\partial_{z}\left(\boldsymbol{A}_{l}^{\dagger} \boldsymbol{A}_{m}^{*}\right)\left(\boldsymbol{A}_{n}^{T} \boldsymbol{A}_{p}\right)= & i\left(\beta_{l}+\beta_{m}-\beta_{n}-\beta_{p}\right)\left(\boldsymbol{A}_{l}^{\dagger} \boldsymbol{A}_{m}^{*}\right)\left(\boldsymbol{A}_{n}^{T} \boldsymbol{A}_{p}\right)+i\left(\boldsymbol{A}_{l}^{\dagger} \mathbf{D}^{\dagger} \boldsymbol{A}_{m}^{*}\right)\left(\boldsymbol{A}_{n}^{T} \boldsymbol{A}_{p}\right) \\
& +i\left(\boldsymbol{A}_{l}^{\dagger} \mathbf{D}^{*} \boldsymbol{A}_{m}^{*}\right)\left(\boldsymbol{A}_{n}^{T} \boldsymbol{A}_{p}\right)-i\left(\boldsymbol{A}_{l}^{\dagger} \boldsymbol{A}_{m}^{*}\right)\left(\boldsymbol{A}_{n}^{T} \mathbf{D}^{T} \boldsymbol{A}_{p}\right)-i\left(\boldsymbol{A}_{l}^{\dagger} \boldsymbol{A}_{m}^{*}\right)\left(\boldsymbol{A}_{n}^{T} \mathbf{D} \boldsymbol{A}_{p}\right) \\
& +i \gamma Y_{l m n p}^{(1)} .
\end{aligned}
$$

We note that $\boldsymbol{A}_{l}^{\dagger} \mathbf{D}^{\dagger} \boldsymbol{A}_{m}^{*}+\boldsymbol{A}_{l}^{\dagger} \mathbf{D}^{*} \boldsymbol{A}_{m}^{*}=2 \sum_{j \in\{1,3\}} \nu_{j} \boldsymbol{A}_{l}^{\dagger} \boldsymbol{\sigma}_{j} \boldsymbol{A}_{m}^{*}$. We follow the procedure outlined above in sections IX A-IX B. Using the Furutsu-Novikov theorem and assuming $\sigma_{\beta} l_{\beta} \ll 1$ and $l_{\beta} \ll L_{n l}$ we obtain

$$
\frac{\delta \boldsymbol{A}_{l}^{\dagger} \boldsymbol{\sigma}_{j} \boldsymbol{A}_{m}^{*} \boldsymbol{A}_{n}^{T} \boldsymbol{A}_{p}(z)}{\delta \nu_{j}\left(z^{\prime}\right)}=2 i\left[\boldsymbol{A}_{l}^{\dagger} \boldsymbol{A}_{m}^{*} \boldsymbol{A}_{n}^{T} \boldsymbol{A}_{p}\left(z^{\prime}\right)-\boldsymbol{A}_{l}^{\dagger} \boldsymbol{\sigma}_{j} \boldsymbol{A}_{m}^{*} \boldsymbol{A}_{n}^{T} \boldsymbol{\sigma}_{j} \boldsymbol{A}_{p}\left(z^{\prime}\right)\right] \exp \left[i\left(\beta_{l}+\beta_{m}-\beta_{n}-\beta_{p}\right)\left(z-z^{\prime}\right)\right]
$$

and therefore

$$
\begin{aligned}
\partial_{z}\left\langle\left(\boldsymbol{A}_{l}^{\dagger} \boldsymbol{A}_{m}^{*}\right)\left(\boldsymbol{A}_{n}^{T} \boldsymbol{A}_{p}\right)\right\rangle= & \left(-4 \Delta \beta+i\left(\beta_{l}+\beta_{m}-\beta_{n}-\beta_{p}\right)\right)\left\langle\left(\boldsymbol{A}_{l}^{\dagger} \boldsymbol{A}_{m}^{*}\right)\left(\boldsymbol{A}_{n}^{T} \boldsymbol{A}_{p}\right)\right\rangle+i \gamma\left\langle Y_{l m n p}^{(1)}\right\rangle \\
& +2 \Delta \beta \sum_{j \in\{1,3\}}\left\langle\left(\boldsymbol{A}_{l}^{\dagger} \boldsymbol{\sigma}_{j} \boldsymbol{A}_{m}^{*}\right)\left(\boldsymbol{A}_{n}^{T} \boldsymbol{\sigma}_{j} \boldsymbol{A}_{p}\right)\right\rangle .
\end{aligned}
$$

The last term can also be written as:

$$
\sum_{j \in\{1,3\}}\left\langle\left(\boldsymbol{A}_{l}^{\dagger} \boldsymbol{\sigma}_{j} \boldsymbol{A}_{m}^{*}\right)\left(\boldsymbol{A}_{n}^{T} \boldsymbol{\sigma}_{j} \boldsymbol{A}_{p}\right)\right\rangle=\left\langle\left(\boldsymbol{A}_{l}^{\dagger} \boldsymbol{A}_{n}\right)\left(\boldsymbol{A}_{m}^{\dagger} \boldsymbol{A}_{p}\right)\right\rangle+\left\langle\left(\boldsymbol{A}_{l}^{\dagger} \boldsymbol{\sigma}_{2} \boldsymbol{A}_{n}\right)\left(\boldsymbol{A}_{m}^{\dagger} \boldsymbol{\sigma}_{2} \boldsymbol{A}_{p}\right)\right\rangle .
$$

The analysis reveals that the terms involving the dissipation that are proportional to $\Delta \beta$ in (62) essentially vanish. Indeed, using the factorizability property of statistical Gaussian fields to split the fourth-order moments into products of second-order moments, then we get $\left\langle\left(\boldsymbol{A}_{l}^{\dagger} \boldsymbol{A}_{m}^{*}\right)\left(\boldsymbol{A}_{n}^{T} \boldsymbol{A}_{p}\right)\right\rangle=\frac{1}{2} w_{l} w_{m}\left(\delta_{l n}^{K} \delta_{m p}^{K}+\delta_{l p}^{K} \delta_{m n}^{K}\right)$ and $\sum_{j \in\{1,3\}}\left\langle\left(\boldsymbol{A}_{l}^{\dagger} \boldsymbol{\sigma}_{j} \boldsymbol{A}_{m}^{*}\right)\left(\boldsymbol{A}_{n}^{T} \boldsymbol{\sigma}_{j} \boldsymbol{A}_{p}\right)\right\rangle=w_{l} w_{m}\left(\delta_{l n}^{K} \delta_{m p}^{K}+\delta_{l p}^{K} \delta_{m n}^{K}\right)$. This shows that, to leading order, the terms involving the dissipation indeed compensate with each other.

2. Computation of the moment $\left\langle\left(\boldsymbol{A}_{n}^{T} \boldsymbol{A}_{m}^{*}\right)\left(\boldsymbol{A}_{l}^{\dagger} \boldsymbol{A}_{p}\right)\right\rangle$

The equation (57) for the evolution of the product of four fields now reads

$$
\begin{aligned}
\partial_{z}\left(\boldsymbol{A}_{n}^{T} \boldsymbol{A}_{m}^{*}\right)\left(\boldsymbol{A}_{l}^{\dagger} \boldsymbol{A}_{p}\right)= & i\left(\beta_{l}+\beta_{m}-\beta_{n}-\beta_{p}\right)\left(\boldsymbol{A}_{n}^{T} \boldsymbol{A}_{m}^{*}\right)\left(\boldsymbol{A}_{l}^{\dagger} \boldsymbol{A}_{p}\right)+i\left(\boldsymbol{A}_{n}^{T} \boldsymbol{A}_{m}^{*}\right)\left(\boldsymbol{A}_{l}^{\dagger} \mathbf{D}^{\dagger} \boldsymbol{A}_{p}\right) \\
& +i\left(\boldsymbol{A}_{n}^{T} \mathbf{D}^{*} \boldsymbol{A}_{m}^{*}\right)\left(\boldsymbol{A}_{l}^{\dagger} \boldsymbol{A}_{p}\right)-i\left(\boldsymbol{A}_{n}^{T} \mathbf{D}^{T} \boldsymbol{A}_{m}^{*}\right)\left(\boldsymbol{A}_{l}^{\dagger} \boldsymbol{A}_{p}\right)-i\left(\boldsymbol{A}_{n}^{T} \boldsymbol{A}_{m}^{*}\right)\left(\boldsymbol{A}_{l}^{\dagger} \mathbf{D} \boldsymbol{A}_{p}\right) \\
& +i \gamma Y_{l m n p}^{(2)} .
\end{aligned}
$$

Since the random matrix is Hermitian $\left(\mathbf{D}^{\dagger}=\mathbf{D}\right)$, the expression can be reduced

$$
\partial_{z}\left(\boldsymbol{A}_{n}^{T} \boldsymbol{A}_{m}^{*}\right)\left(\boldsymbol{A}_{l}^{\dagger} \boldsymbol{A}_{p}\right)=i\left(\beta_{l}+\beta_{m}-\beta_{n}-\beta_{p}\right)\left(\boldsymbol{A}_{n}^{T} \boldsymbol{A}_{m}^{*}\right)\left(\boldsymbol{A}_{l}^{\dagger} \boldsymbol{A}_{p}\right)+i \gamma Y_{l m n p}^{(2)} .
$$

The fourth-order moment $\left\langle\left(\boldsymbol{A}_{n}^{T} \boldsymbol{A}_{m}^{*}\right)\left(\boldsymbol{A}_{l}^{\dagger} \boldsymbol{A}_{p}\right)\right\rangle$ then evolves as in the absence of any disorder $(\Delta \beta=0)$.

\section{Impact of a partially correlated noise model of disorder on the kinetic equation}

In the partially correlated model of disorder, all degenerate modes experience the same realization of disorder. Groups of degenerate modes with the same reduced eigenvalue $\beta$ are indexed by $p$, the modes within the $p$ th group 
are indexed by $(p, j)$, and the linear polarization components of the $(p, j)$-th mode are $\boldsymbol{A}_{p j}$. The structural disorder induces a linear random coupling between the modes of different groups, as described by the $2 \times 2$ matrix $\mathbf{D}_{p}(z)=$ $\sum_{l=0}^{3} \nu_{p, l}(z) \boldsymbol{\sigma}_{l}$ where $p$ labels the mode group number, i.e., degenerate modes that belong to the same group experience the same noise through the random process $\nu_{p, l}(z)$ (this notation should not be confused with the decorrelated model of disorder where $p$ labels individual modes). The evolutions of the modal components are governed by

$$
i \partial_{z} \boldsymbol{A}_{p j}=\beta_{p} \boldsymbol{A}_{p j}+\mathbf{D}_{p}(z) \boldsymbol{A}_{p j}-\gamma \boldsymbol{P}_{p j}(\boldsymbol{A}) .
$$

We look at the second-order moments $\left\langle\boldsymbol{A}_{p j}^{*} \boldsymbol{A}_{q l}^{T}\right\rangle$. We follow the procedure outlined above in section IX A by using the Furutsu-Novikov theorem with $\sigma_{\beta} l_{\beta} \ll 1$ and $l_{\beta} \ll L_{n l}$.

Considering non-degenerate modes $(p \neq q)$, we obtain

$$
\left\langle\boldsymbol{A}_{p j}^{*} \boldsymbol{A}_{q l}^{T}\right\rangle(z)=\frac{i \gamma}{4 \Delta \beta-i\left(\beta_{p}-\beta_{q}\right)}\left\langle\boldsymbol{P}_{p j}(\boldsymbol{A})^{*} \boldsymbol{A}_{q l}^{T}-\boldsymbol{A}_{p j}^{*} \boldsymbol{P}_{q l}(\boldsymbol{A})^{T}\right\rangle .
$$

In the regime $L_{d}=1 / \Delta \beta \ll L_{n l}$ the correlation is vanishing small, $\left\langle\boldsymbol{A}_{p j}^{*} \boldsymbol{A}_{q l}^{T}\right\rangle(z) \simeq 0$, as in the model of decorrelated disorder.

Let us now consider correlations among the orthogonal polarization components of a mode $(p=q$ and $j=l)$, then we find as before

$$
\left\langle\boldsymbol{A}_{p j}^{*} \boldsymbol{A}_{p j}^{T}\right\rangle(z)=w_{p, j j}(z) \boldsymbol{\sigma}_{0} .
$$

We consider correlations among distinct degenerate modes $(p=q$ and $j \neq l)$. We define the two Hermitian matrices $\mathbf{W}_{p, j l}=\frac{1}{2}\left(\left\langle\boldsymbol{A}_{p j}^{*} \boldsymbol{A}_{p l}^{T}\right\rangle+\left\langle\boldsymbol{A}_{p l}^{*} \boldsymbol{A}_{p j}^{T}\right\rangle\right)$ and $\tilde{\mathbf{W}}_{p, j l}=\frac{i}{2}\left(\left\langle\boldsymbol{A}_{p j}^{*} \boldsymbol{A}_{p l}^{T}\right\rangle-\left\langle\boldsymbol{A}_{p l}^{*} \boldsymbol{A}_{p j}^{T}\right\rangle\right)$ and then by carrying out the same calculations as in the case $p=q$ and $j=l$, we find

$$
\left\langle\boldsymbol{A}_{p j}^{*} \boldsymbol{A}_{p l}^{T}\right\rangle(z)=w_{p, j l}(z) \boldsymbol{\sigma}_{0} .
$$

The coefficients $w_{p, j l}$ satisfy

$$
\begin{aligned}
\partial_{z} w_{p, j l} & =\frac{\gamma}{2} \operatorname{Im}\left\langle\boldsymbol{P}_{p j}(\boldsymbol{A})^{\dagger} \boldsymbol{A}_{p l}+\boldsymbol{P}_{p l}(\boldsymbol{A})^{\dagger} \boldsymbol{A}_{p j}\right\rangle=\frac{1}{6} \gamma\left\langle X_{p, j l}^{(1)}+X_{p, l j}^{(1)}\right\rangle+\frac{1}{3} \gamma\left\langle X_{p, j l}^{(2)}+X_{p, l j}^{(2)}\right\rangle, \\
X_{p, j l}^{(1)} & =\operatorname{Im}\left\{\sum_{p_{1} j_{1}, p_{2} j_{2}, p_{3} j_{3}} S_{p l, p_{1} j_{1}, p_{2} j_{2}, p_{3} j_{3}}^{*}\left(\boldsymbol{A}_{p_{1} j_{1}}^{\dagger} \boldsymbol{A}_{p_{2} j_{2}}^{*}\right)\left(\boldsymbol{A}_{p_{3} j_{3}}^{T} \boldsymbol{A}_{p j}\right)\right\}, \\
X_{p, j l}^{(2)} & =\operatorname{Im}\left\{\sum_{p_{1} j_{1}, p_{2} j_{2}, p_{3} j_{3}} S_{p l, p_{1} j_{1}, p_{2} j_{2}, p_{3} j_{3}}^{*}\left(\boldsymbol{A}_{p_{3} j_{3}}^{T} \boldsymbol{A}_{p_{2} j_{2}}^{*}\right)\left(\boldsymbol{A}_{p_{1} j_{1}}^{\dagger} \boldsymbol{A}_{p j}\right)\right\} .
\end{aligned}
$$

Let us look at the fourth-order moments $\left\langle\left(\boldsymbol{A}_{p_{1} j_{1}}^{\dagger} \boldsymbol{A}_{p_{2} j_{2}}^{*}\right)\left(\boldsymbol{A}_{p_{3} j_{3}}^{T} \boldsymbol{A}_{p_{4} j_{4}}\right)\right\rangle$ or $\left\langle\left(\boldsymbol{A}_{p_{1} j_{1}}^{T} \boldsymbol{A}_{p_{2} j_{2}}^{*}\right)\left(\boldsymbol{A}_{p_{3} j_{3}}^{\dagger} \boldsymbol{A}_{p_{4} j_{4}}\right)\right\rangle$.

There are different types of such fourth-order moments that depend on the specific modes that they involve. Almost all of them satisfy an evolution equation with damping proportional to $\Delta \beta$, with different coefficients in front of $\Delta \beta$ that depend on the number of equal indices. These terms are of the same form as those obtained by considering the decorrelated model of disorder, see section IX B. There are special cases when $p_{j}$ are equal by pairs (e.g. $p_{1}=p_{2}$ and $\left.p_{3}=p_{4}\right)$ where

$$
\partial_{z}\left\langle\left(\boldsymbol{A}_{p_{1} j_{1}}^{T} \boldsymbol{A}_{p_{1} j_{2}}^{*}\right)\left(\boldsymbol{A}_{p_{3} j_{3}}^{\dagger} \boldsymbol{A}_{p_{3} j_{4}}\right)\right\rangle=i \gamma Y_{p_{3} j_{3}, p_{1} j_{2}, p_{1} j_{1}, p_{3} j_{4}}^{(2)},
$$

which shows that there is no damping. From the expression of $Y^{(2)}$ (see Eq.(58)), and the evaluation of its expectation in terms of the $w_{p, j l}$ according to the Gaussian rule for sixth-order moments, such terms induce a reversible exchange of energy between the modes within each group.

\section{APPENDIX B: KINETIC EQUATIONS WITHOUT DISORDER}

\section{A. Continuous and discrete wave turbulence regimes}

The continuous kinetic equation describing weak turbulence in the presence of a parabolic potential was derived in Ref.[17], see Eq.(17-18). The presence of the factor $1 / \beta_{0}^{6}$ in the kinetic equation is due to the introduction of 
a continuous frequency variable $\kappa=\beta_{0}\left(p_{x}, p_{y}\right)$, as discussed through the continuous kinetic Eq.(22) above. The characteristic scale of evolution of the modal components then reads $L_{k i n}^{\text {ord }} \sim \beta_{0} L_{n l}^{2} / \bar{S}_{l m n p}^{2}$, as given in Eq. $(32)$.

Let us now consider the discrete wave turbulence regime without disorder. By ignoring polarization effects $\left(\boldsymbol{A}_{p} \rightarrow\right.$ $\left.A_{p, 1}\right)$, the set of closed equations for the second- and fourth-order moments read [17]:

$$
\begin{aligned}
\partial_{z} n_{p} & =-\gamma i \sum_{l, m, n} S_{l m n p} \tilde{J}_{l m n p} \exp \left(i \Delta \omega_{l m n p} z\right)+\gamma i \sum_{l, m, n} S_{l m n p}^{*} \tilde{J}_{l m n p}^{*} \exp \left(-i \Delta \omega_{l m n p} z\right) \\
\partial_{z} \tilde{J}_{l m n p} & =2 i \gamma S_{l m n p}^{*} M_{l m n p}(\boldsymbol{n}) \exp \left(-i \Delta \omega_{l m n p} z\right)
\end{aligned}
$$

where $M_{l m n p}(\boldsymbol{n})=n_{l} n_{m} n_{p}+n_{l} n_{m} n_{n}-n_{n} n_{p} n_{m}-n_{n} n_{p} n_{l}, J_{l m n p}(z)=\left\langle A_{l}^{*} A_{m}^{*} A_{n} A_{p}\right\rangle$ is the fourth order moment with $\tilde{J}_{l m n p}=J_{l m n p} \exp \left(-i \Delta \omega_{l m n p} z\right)$ and $\Delta \omega_{l m n p}=\beta_{l}+\beta_{m}-\beta_{n}-\beta_{p}\left(\beta_{p}=\beta_{0}\left(1+p_{x}+p_{y}\right)\right)$. Note that for the sake of simplicity and clarity, we do not write in Eq.(64) the contribution that leads to the second term in the rhs of the kinetic equations (24) or (29), whose main effect is to enforce energy equipartition within groups of degenerate modes.

Recalling that $\beta_{0} L_{n l} \gg 1$, we make use of the standard homogenization theorem to obtain the effective equations for $z \sim L_{n l}$ :

$$
\begin{aligned}
\partial_{z} n_{p} & =i \gamma \sum_{l, m, n} \delta^{K}\left(\Delta \omega_{l m n p}\right)\left(-S_{l m n p} \tilde{J}_{l m n p}+S_{l m n p}^{*} \tilde{J}_{l m n p}^{*}\right) \\
\partial_{z} \tilde{J}_{l m n p} & =2 i \gamma S_{l m n p}^{*} M_{l m n p}(\boldsymbol{n}) \delta^{K}\left(\Delta \omega_{l m n p}\right)
\end{aligned}
$$

where we recall that $\delta^{K}\left(\Delta \omega_{l m n p}\right)=1$ if $\Delta \omega_{l m n p}=0$, and zero otherwise. Accordingly, one obtains an equation for the modal components that is formally reversible in $z$. This equation is valid for propagation lengths of the order of $L_{n l}$. For longer propagation lengths, corrective terms must be taken into account [132], showing that the characteristic length of evolution of the modal components $n_{p}(z)$ scales as in the continuous case $L_{k i n}^{\text {ord }} \sim \beta_{0} L_{n l}^{2} / \bar{S}_{l m n p}^{2}$ (up to corrective prefactors).

\section{B. Impact of a perturbation of the dispersion relation}

In this paragraph we consider the impact of a perturbation of the dispersion relation $\tilde{\beta}_{p}=\beta_{p}+b_{p}\left(\right.$ with $\left.b_{p} / \beta_{p} \ll 1\right)$ on the kinetic equation without disorder. This issue is addressed in section VIC in the presence of disorder. The equations for the second- and fourth-order moments (64-65) take the form

$$
\begin{aligned}
\partial_{z} n_{p} & =2 \gamma \sum_{l, m, n} \operatorname{Im}\left[S_{l m n p} J_{l m n p}(z)\right] \\
J_{l m n p}(z) & =J_{l m n p}(0) \exp \left(i \Delta \tilde{\omega}_{l m n p} z\right)+2 i \gamma \int_{0}^{z} S_{l m n p}^{*} M_{l m n p}(\boldsymbol{n}(\zeta)) \exp \left(i \Delta \tilde{\omega}_{l m n p}(z-\zeta)\right) d \zeta
\end{aligned}
$$

where $\Delta \tilde{\omega}_{l m n p}=\tilde{\beta}_{l}+\tilde{\beta}_{m}-\tilde{\beta}_{n}-\tilde{\beta}_{p}$. In the following we neglect the initial condition $J_{l m n p}(0)$. This can be justified when the initial condition exhibits random phases among the modes, but not when the initial condition is a coherent beam as in usual experiments of beam self-cleaning. Resonances that are exact at leading order $\left(\Delta \omega_{l m n p}=\beta_{l}+\beta_{m}-\beta_{n}-\beta_{p}=0\right)$ exhibit a residual non-resonant contribution, i.e., $\Delta \tilde{\omega}_{l m n p}=\tilde{\beta}_{l}+\tilde{\beta}_{m}-\tilde{\beta}_{n}-\tilde{\beta}_{p}=\Delta b_{l m n p}$ with $\Delta b_{l m n p}=b_{l}+b_{m}-b_{n}-b_{p}$. Since $\beta_{0} L_{n l} \gg 1$ only leading order exact resonances can contribute:

$$
\partial_{z} n_{p}=4 \gamma^{2} \sum_{l, m, n}\left|S_{l m n p}\right|^{2} \delta^{K}\left(\Delta \omega_{l m n p}\right) \int_{0}^{z} \cos \left(\Delta b_{l m n p}(z-\zeta)\right) M_{l m n p}(\boldsymbol{n}(\zeta)) d \zeta .
$$

If the resonances are approximate, then the quartets $\{l, m, n, p\}$ such that $\left|\Delta b_{l m n p}\right| L_{k i n}^{p e r t} \sim 1$ contribute to the convolution in (70), where $L_{k i n}^{\text {pert }}$ is the characteristic evolution length of the moments in the presence of the perturbation $b_{p}$. Those quartets for which $\left|\Delta b_{\text {lmnp }}\right| L_{k i n}^{\text {pert }} \gg 1$ average out to zero. Those quartets for which $\left|\Delta b_{l m n p}\right| L_{k i n}^{\text {pert }} \ll 1$ should give rise to reversible equations but we neglect them because we assume here that there are many more quasi-resonances than resonances. Since quasi-resonances are more important than exact resonances we can take the continuous limit with $\sin \left(\Delta b_{l m n p} z\right) / \Delta b_{l m n p} \rightarrow \pi \delta\left(\Delta b_{l m n p}\right)$ for $z \gg \pi / \min \left(\Delta b_{l m n p}\right)$, where $\delta(x)$ denotes the Dirac distribution. Note that the passage to the continuous limit can be justified for a perturbation that removes mode degeneracies, such as the truncation of the parabolic potential (see Fig. 5 in [17]), but not for the perturbation induced by the Helmholtz equation that does not remove degeneracies, see section VI C. According to the continuous kinetic equation, the characteristic evolution length of the kinetics scales as $L_{k i n}^{p e r t} \sim b_{p} L_{n l}^{2} / \bar{S}_{l m n p}^{2}$. 


\section{APPENDIX C: DERIVATION OF THE DISCRETE KINETIC EQUATION WITH STRONG DISORDER}

\section{A. First order moments}

The model of strong disorder is defined from the $2 N_{*} \times 2 N_{*}$ random matrix $\mathbf{D}$ in Eq.(35). The matrices $\mathbf{H}^{q r}, \mathbf{K}^{q r}$, $\mathbf{J}^{q}$ are defined by:

$$
\left(\mathbf{H}^{q r}\right)_{j l}=\frac{1}{\sqrt{2}}\left\{\begin{array}{ll}
1 & \text { if }(j, l)=(q, r) \\
1 & \text { if }(j, l)=(r, q) \\
0 & \text { otherwise }
\end{array} \quad\left(\mathbf{K}^{q r}\right)_{j l}=\frac{1}{\sqrt{2}}\left\{\begin{array}{l}
i \quad \text { if }(j, l)=(q, r) \\
-i \text { if }(j, l)=(r, q) \\
0 \quad \text { otherwise }
\end{array} \quad\left(\mathbf{J}^{q}\right)_{j l}= \begin{cases}1 & \text { if }(j, l)=(q, q) \\
0 & \text { otherwise }\end{cases}\right.\right.
$$

We have the identity

$$
\sum_{q<r}\left(\left(\mathbf{H}^{q r}\right)^{2}+\left(\mathbf{K}^{q r}\right)^{2}\right)+\sum_{q}\left(\mathbf{J}^{q}\right)^{2}=2 N_{*} \mathbf{I}
$$

In these conditions $i(\boldsymbol{\beta}+\mathbf{D}(z))$ generates a shifted unitary Brownian motion $\mathbf{U}(z)$,

$$
\partial_{z} \mathbf{U}=i(\boldsymbol{\beta}+\mathbf{D}(z)) \mathbf{U}, \quad \mathbf{U}(z=0)=\mathbf{I},
$$

which is a random diffusion on the group of unitary matrices whose stationary distribution is the uniform (Haar) measure. We have $\left\|\mathbf{U}(z) \boldsymbol{A}_{0}\right\|=\left\|\boldsymbol{A}_{0}\right\|$ for any constant vector $\boldsymbol{A}_{0}$.

The evolution of the average of the unitary matrix $\mathbf{U}$ is governed by

$$
\frac{d \mathbb{E}[\mathbf{U}(z)]}{d z}=i \boldsymbol{\beta} \mathbb{E}[\mathbf{U}(z)]+\frac{i}{\sqrt{2 N_{*}}}\left\{\sum_{q<r} g_{q r}\left(\mathbf{H}^{q r} \mathbb{E}\left[\nu_{q r}(z) \mathbf{U}(z)\right]+\mathbf{K}^{q r} \mathbb{E}\left[\mu_{q r}(z) \mathbf{U}(z)\right]\right)+\sum_{q} g_{q} \mathbf{J}^{q} \mathbb{E}\left[\eta_{q}(z) \mathbf{U}(z)\right]\right\} .
$$

We follow the same procedure as in Appendix A to evaluate the averages by using the Furutsu-Novikov theorem:

$$
\mathbb{E}\left[\nu_{q r}(z) \mathbf{U}(z)\right]=\int_{0}^{z} \mathbb{E}\left[\frac{\delta \mathbf{U}(z)}{\delta \nu_{q r}\left(z^{\prime}\right)}\right] \sigma_{\beta}^{2} \mathcal{R}\left(\frac{z-z^{\prime}}{l_{\beta}}\right) d z^{\prime} .
$$

The variational derivative satisfies for $z>z^{\prime}$ :

$$
\partial_{z} \frac{\delta \mathbf{U}(z)}{\delta \nu_{q r}\left(z^{\prime}\right)}=i \boldsymbol{\beta} \frac{\delta \mathbf{U}(z)}{\delta \nu_{q r}\left(z^{\prime}\right)}+\mathbf{D}(z) \frac{\delta \mathbf{U}(z)}{\delta \nu_{q r}\left(z^{\prime}\right)},
$$

with

$$
\left.\frac{\delta \mathbf{U}(z)}{\delta \nu_{q r}\left(z^{\prime}\right)}\right|_{z=z^{\prime}}=\frac{i}{\sqrt{2 N_{*}}} g_{q r} \mathbf{H}^{q r} \mathbf{U}\left(z^{\prime}\right)
$$

Therefore, if $\sigma_{\beta} l_{\beta} \ll 1$, we get the simplified equation

$$
\begin{aligned}
\frac{\delta \mathbf{U}(z)}{\delta \nu_{q r}\left(z^{\prime}\right)} & =\frac{i}{\sqrt{2 N_{*}}} g_{q r} \exp \left(i \boldsymbol{\beta}\left(z-z^{\prime}\right)\right) \mathbf{H}^{q r} \mathbf{U}\left(z^{\prime}\right), \\
\left(\frac{i}{\sqrt{2 N_{*}}} \mathbf{H}^{q r} \frac{\delta \mathbf{U}(z)}{\delta \nu_{q r}\left(z^{\prime}\right)}\right)_{j l} & =-\frac{1}{4 N_{*}} g_{q r}^{2}\left(\delta_{q j}^{K} \exp \left(i \beta_{r}\left(z-z^{\prime}\right)\right)+\delta_{r j}^{K} \exp \left(i \beta_{q}\left(z-z^{\prime}\right)\right)\right) U_{j l}\left(z^{\prime}\right) \\
& =-\frac{1}{4 N_{*}} g_{q r}^{2}\left(\delta_{q j}^{K} \exp \left(i\left(\beta_{r}-\beta_{q}\right)\left(z-z^{\prime}\right)\right)+\delta_{r j}^{K} \exp \left(i\left(\beta_{q}-\beta_{r}\right)\left(z-z^{\prime}\right)\right)\right) U_{j l}(z),
\end{aligned}
$$

and

$$
\begin{aligned}
\left(\frac{i}{\sqrt{2 N_{*}}} \mathbf{H}^{q r} \mathbb{E}\left[\nu_{q r}(z) \mathbf{U}(z)\right]\right)_{j l}=- & \frac{1}{2 N} g_{q r}^{2} \int_{0}^{z} \sigma_{\beta}^{2} \mathcal{R}\left(\frac{z-z^{\prime}}{l_{\beta}}\right)\left(\delta_{q j}^{K} \exp \left(i\left(\beta_{r}-\beta_{q}\right)\left(z-z^{\prime}\right)\right)\right. \\
& \left.+\delta_{r j}^{K} \exp \left(i\left(\beta_{q}-\beta_{r}\right)\left(z-z^{\prime}\right)\right)\right) d z^{\prime} U_{j l}(z) .
\end{aligned}
$$


We get similarly

$$
\begin{aligned}
\left(\frac{i}{\sqrt{2 N_{*}}} \mathbf{K}^{q r} \mathbb{E}\left[\mu_{q r}(z) \mathbf{U}(z)\right]\right)_{j l}=- & \frac{1}{4 N_{*}} g_{q r}^{2} \int_{0}^{z} \sigma_{\beta}^{2} \mathcal{R}\left(\frac{z-z^{\prime}}{l_{\beta}}\right)\left(\delta_{q j}^{K} \exp \left(i\left(\beta_{r}-\beta_{q}\right)\left(z-z^{\prime}\right)\right)\right. \\
& \left.+\delta_{r j}^{K} \exp \left(i\left(\beta_{q}-\beta_{r}\right)\left(z-z^{\prime}\right)\right)\right) d z^{\prime} U_{j l}(z), \\
\left(\frac{i}{\sqrt{2 N_{*}}} \mathbf{J}^{q} \mathbb{E}\left[\eta_{q}(z) \mathbf{U}(z)\right]\right)_{j l}= & -\frac{1}{2 N_{*}} g_{q}^{2} \int_{0}^{z} \sigma_{\beta}^{2} \mathcal{R}\left(\frac{z-z^{\prime}}{l_{\beta}}\right) d z^{\prime} \mathbf{1}_{q}(j) U_{j l}(z) .
\end{aligned}
$$

Therefore, using $z \gg l_{\beta}$,

$$
\frac{d \mathbb{E}[\mathbf{U}(z)]}{d z}=i \boldsymbol{\beta} \mathbb{E}[\mathbf{U}(z)]-\mathbf{Q} \mathbb{E}[\mathbf{U}(z)]
$$

with $\mathbf{Q}$ the diagonal matrix with diagonal coefficients

$$
Q_{m m}=\frac{\Delta \beta}{2 N_{*}} \int_{0}^{\infty} \sigma_{\beta}^{2} \mathcal{R}(s)\left(g_{m}^{2}+\sum_{p>m} g_{m p}^{2} \exp \left(i\left(\beta_{p}-\beta_{m}\right) l_{\beta} s\right)+\sum_{p<m} g_{p m}^{2} \exp \left(i\left(\beta_{p}-\beta_{m}\right) l_{\beta} s\right) d s\right) .
$$

The real part of $Q_{m m}$ is positive (because it is the sum of Fourier coefficients of $\mathcal{R}$, which are non-negative by Bochner's theorem), which means that the mean wave mode amplitudes decay exponentially with the decay rate $1 / Q_{m m}$.

\section{B. Second order moments}

We can proceed in the same way for the second-order moment. Let us denote $a_{p}(z)=\left(\mathbf{U}(z) \boldsymbol{A}_{0}\right)_{p}$. We have

$$
\frac{d a_{n}}{d z}=i \beta_{n} a_{n}+\frac{i}{\sqrt{4 N_{*}}}\left\{\sum_{q<n} g_{q n} \nu_{q n} a_{q}+\sum_{q>n} g_{n q} \nu_{n q} a_{q}-i \sum_{q<n} g_{q n} \mu_{q n} a_{q}+i \sum_{q>n} g_{n q} \mu_{n q} a_{q}+\sqrt{2} g_{n} \eta_{n} a_{n}\right\},
$$

and therefore

$$
\begin{aligned}
\frac{d\left|a_{n}\right|^{2}}{d z}= & \frac{i}{\sqrt{2 N}}\left\{\sum_{q<n} g_{q n} \nu_{q n}\left(a_{q} a_{n}{ }^{*}-a_{n} a_{q}{ }^{*}\right)+\sum_{q>n} g_{n q} \nu_{n q}\left(a_{q} a_{n}{ }^{*}-a_{n} a_{q}{ }^{*}\right)\right. \\
& \left.-i \sum_{q<n} g_{q n} \mu_{q n}\left(a_{q} a_{n}{ }^{*}+a_{n} a_{q}{ }^{*}\right)+i \sum_{q>n} g_{n q} \mu_{n q}\left(a_{q} a_{n}{ }^{*}+a_{n} a_{q}{ }^{*}\right)\right\} .
\end{aligned}
$$

By Furutsu-Novikov formula, we have for $q<n$

$$
\mathbb{E}\left[\nu_{q n}(z) a_{n} a_{q}{ }^{*}(z)\right]=\int_{0}^{z} \mathbb{E}\left[\frac{\delta a_{n} a_{q}{ }^{*}(z)}{\delta \nu_{q n}\left(z^{\prime}\right)}\right] \sigma_{\beta}^{2} \mathcal{R}\left(\frac{z-z^{\prime}}{l_{\beta}}\right) d z^{\prime} .
$$

The variational derivative $\frac{\delta a_{n} a_{q}{ }^{*}(z)}{\delta \nu_{q n}\left(z^{\prime}\right)}$, for $q<n$, satisfies for $z>z^{\prime}$ :

$$
\partial_{z} \frac{\delta a_{n} a_{q}^{*}(z)}{\delta \nu_{q n}\left(z^{\prime}\right)}=i\left(\beta_{n}-\beta_{q}\right) \frac{\delta a_{n} a_{q}{ }^{*}(z)}{\delta \nu_{q n}\left(z^{\prime}\right)}+\cdots
$$

with

$$
\left.\frac{\delta a_{n} a_{q}{ }^{*}(z)}{\delta \nu_{q n}\left(z^{\prime}\right)}\right|_{z=z^{\prime}}=\frac{i}{\sqrt{4 N_{*}}} g_{q n}\left(\left|a_{q}\right|^{2}-\left|a_{n}\right|^{2}\right)\left(z^{\prime}\right) .
$$

Therefore, if $\sigma_{\beta} l_{\beta} \ll 1$, then we get

$$
\frac{\delta a_{n} a_{q}{ }^{*}(z)}{\delta \nu_{q n}\left(z^{\prime}\right)}=\frac{i}{\sqrt{4 N_{*}}} g_{q n}\left(\left|a_{q}\right|^{2}-\left|a_{n}\right|^{2}\right)\left(z^{\prime}\right) \exp \left(i\left(\beta_{n}-\beta_{q}\right)\left(z-z^{\prime}\right)\right),
$$

and we have similar expressions for the other terms. Consequently, $w_{m}=\mathbb{E}\left[\left|a_{m}\right|^{2}\right]$ satisfy Eq.(36):

$$
\frac{d w_{m}}{d z}=2 \sigma_{\beta}^{2} \sum_{p=0}^{2 N_{*}-1} g_{m p}^{2} \int_{0}^{z}\left(w_{p}-w_{m}\right)\left(z^{\prime}\right) \mathcal{R}\left(\frac{z-z^{\prime}}{l_{\beta}}\right) \cos \left(\left(\beta_{p}-\beta_{m}\right)\left(z-z^{\prime}\right)\right) d z^{\prime} .
$$


[1] S. Pitaevskii, L. Stringari, Bose-Einstein Condensation (Oxford Science Publications, 2003).

[2] I. Carusotto, C. Ciuti, Quantum fluids of light, Rev. Modern Phys. 85299 (2013).

[3] J. Klaers, J. Schmitt, F. Vewinger, M. Weitz, BoseEinstein condensation of photons in an optical microcavity, Nature 468, 545 (2010).

[4] R. Weill, A. Bekker, B. Levit, B. Fischer, Bose-Einstein condensation of photons in an erbium-ytterbium codoped fiber cavity, Nature Comm. 10, 1-6 (2019).

[5] S. Dyachenko, A.C. Newell, A. Pushkarev, and V.E. Zakharov, Optical turbulence: Weak turbulence, condensates and collapsing filaments in the nonlinear Schrödinger equation, Physica D 57, 96-160 (1992).

[6] A.C. Newell, S. Nazarenko, L. Biven, Wave turbulence and intermittency, Physica D 152, 520 (2001).

[7] S. Nazarenko, Wave Turbulence (Springer, Lectures Notes in Physics, 2011).

[8] A.C. Newell, B. Rumpf, Wave Turbulence, Annu. Rev. Fluid Mech. 43, 59 (2011).

[9] N.G. Berloff and B.V. Svistunov, Scenario of strongly nonequilibrated Bose-Einstein condensation, Phys. Rev. A 66, 013603 (2002).

[10] C. Connaughton, C. Josserand, A. Picozzi, Y. Pomeau, S. Rica, Condensation of classical nonlinear waves, Phys. Rev. Lett. 95, 263901 (2005).

[11] S. Nazarenko, M. Onorato, Wave turbulence and vortices in Bose-Einstein condensation, Physica D 219, 1 (2006).

[12] N.G. Berloff, A.J. Youd, Dissipative dynamics of superfluid vortices at nonzero temperatures, Phys. Rev. Lett. 99, 145301 (2007).

[13] G. Düring, A. Picozzi, S. Rica, Breakdown of weakturbulence and nonlinear wave condensation, Physica D 238, 1524 (2009).

[14] H. Salman, N. Berloff, Condensation of classical nonlinear waves in a two-component system, Physica D 238, 1482 (2009).

[15] D. Proment, S. Nazarenko, M. Onorato, Sustained turbulence in the three-dimensional Gross-Pitaevskii model, Physica D 241, 304-314 (2012).

[16] G. Krstulovic and M. Brachet, Dispersive Bottleneck Delaying Thermalization of Turbulent Bose-Einstein Condensates, Phys. Rev. Lett. 106, 115303 (2011).

[17] P. Aschieri, J. Garnier, C. Michel, V. Doya, A. Picozzi, Condensation and thermalization of classsical optical waves in a waveguide, Phys. Rev. A 83, 033838 (2011).

[18] C. Sun, S. Jia, C. Barsi, S. Rica, A. Picozzi, J. Fleischer, Observation of the kinetic condensation of classical waves, Nature Phys. 8, 471 (2012).

[19] P. Suret and S. Randoux, Far field measurement in the focal plane of a lens: a cautionary note, arXiv:1307.5034.

[20] A. Picozzi, J. Garnier, T. Hansson, P. Suret, S. Randoux, G. Millot, D.N. Christodoulides, Optical wave turbulence: Toward a unified nonequilibrium thermodynamic formulation of statistical nonlinear optics, Physics Reports 542, 1-132 (2014).

[21] A. Rückriegel, P. Kopietz, Rayleigh-Jeans condensation of pumped magnons in thin-film ferromagnets, Phys. Rev. Lett. 115, 157203 (2015).
[22] S. Nazarenko, M. Onorato, D. Proment, Bose-Einstein condensation and Berezinskii-Kosterlitz-Thouless transition in the two-dimensional nonlinear Schrödinger model, Phys. Rev. A 90, 013624 (2014).

[23] N. Santic, A. Fusaro, S. Salem, J. Garnier, A. Picozzi, R. Kaiser, Nonequilibrium precondensation of classical waves in two dimensions propagating through atomic vapors, Phys. Rev. Lett. 120, 055301 (2018).

[24] V.E. Zakharov, V.S. L'vov, G. Falkovich, Kolmogorov Spectra of Turbulence I (Springer, Berlin, 1992).

[25] Advances in Wave Turbulence, World Scientific Series on Nonlinear Science Series A, Vol. 83, edited by V.I. Shrira and S. Nazarenko (World Scientific, Singapore, 2013).

[26] A. Chiocchetta, P.E. Larré, I. Carusotto, Thermalization and Bose-Einstein condensation of quantum light in bulk nonlinear media, Europhys. Lett. 115, 24002 (2016).

[27] J. Laurie, U. Bortolozzo, S. Nazarenko, S. Residori, One-dimensional optical wave turbulence: experiment and theory, Physics Reports 514, 121-175 (2012).

[28] C. Conti, M. Leonetti, A. Fratalocchi, L. Angelani, G. Ruocco, Condensation in disordered lasers: Theory, $3 \mathrm{~d}+1$ simulations, and experiments, Phys. Rev. Lett. 101, 143901 (2008).

[29] R. Weill, B. Fischer, O. Gat, Light-mode condensation in actively-mode-locked lasers, Phys. Rev. Lett. 104, 173901 (2010).

[30] N.G. Berloff and J. Keeling, Universality in modelling non-equilibrium polariton condensates, In: A. Bramati, M. Modugno (eds) Physics of Quantum Fluids (Springer Series in Solid-State Sciences, vol 177, Springer, Berlin, Heidelberg, 2013).

[31] G. Oren, A. Bekker, B. Fischer, Classical condensation of light pulses in a loss trap in a laser cavity, Optica 1, 145 (2014).

[32] E. Turitsyna, G. Falkovich, A. El-Taher, X. Shu, P. Harper, S. Turitsyn, Optical turbulence and spectral condensate in long fibre lasers, Proc. R. Soc. A 468, 2145 (2012)

[33] E. Turitsyna, S. Smirnov, S. Sugavanam, N. Tarasov, X. Shu, S. Babin, E. Podivilov, D. Churkin, G. Falkovich, S. Turitsyn, The laminar-turbulent transition in a fibre laser, Nature Photon. 7, 783 (2013).

[34] D. Churkin, I. Kolokolov, E. Podivilov, I. Vatnik, S. Vergeles, I. Terekhov, V. Lebedev, G. Falkovich, M. Nikulin, S. Babin, S. Turitsyn, Wave kinetics of a random fibre laser, Nature Commun. 2, 6214 (2015).

[35] J. Huang, C. Liu, Y. Zhu, S. Masala, E. Alarousu, Y. Han, A. Fratalocchi, Harnessing structural darkness in the visible and infrared wavelengths for a new source of light, Nat. Nanotech. 11, 60 (2015).

[36] M. Sich, J. K. Chana, O. A. Egorov, H. Sigurdsson, I. A. Shelykh, D. V. Skryabin, P. M. Walker, E. Clarke, B. Royall, M. S. Skolnick, and D. N. Krizhanovskii, Transition from propagating polariton solitons to a standing wave condensate induced by interactions, Phys. Rev. Lett. 120, 167402 (2018).

[37] K. Krupa, A. Tonello, A. Barthélémy, V. Couderc, B.M. Shalaby, A. Bendahmane, G. Millot, S. Wabnitz, Observation of geometric parametric instability induced by 
the periodic spatial self-imaging of multimode waves, Phys. Rev. Lett. 116, 183901 (2016).

[38] L.G. Wright, Z. Liu, D.A. Nolan, M.-J. Li, D.N. Christodoulides, F.W. Wise, Self-organized instability in graded-index multimode fibres, Nature Photon. 10, 771 (2016).

[39] K. Krupa, A. Tonello, B.M. Shalaby, M. Fabert, A. Barthélémy, G. Millot, S. Wabnitz, V. Couderc, Spatial beam self-cleaning in multimode fibres, Nature Photon. 11, 237 (2017).

[40] N.B. Terry, T.G. Alley, T.H. Russell, An explanation of SRS beam cleanup in graded-index fibers and the absence of SRS beam cleanup in step-index fibers, Opt. Express 15, 17509 (2007).

[41] A. Fusaro, J. Garnier, K. Krupa, G. Millot, A. Picozzi, Dramatic acceleration of wave condensation mediated by disorder in multimode fibers, Phys. Rev. Lett. 122, 123902 (2019).

[42] E. Podivilov, D. Kharenko, V. Gonta, K. Krupa, O.S. Sidelnikov, S. Turitsyn, M.P. Fedoruk, S.A. Babin, S. Wabnitz, Hydrodynamic 2D turbulence and spatial beam condensation in multimode optical fibers, Phys. Rev. Lett. 122, 103902 (2019).

[43] F.O. Wu, A.U. Hassan, D.N. Christodoulides, Thermodynamic theory of highly multimoded nonlinear optical systems, Nature Photon. (to be published).

[44] I.P. Kaminow, T. Li, A.F. Willner, Optical Fiber Telecommunications, Systems and Networks (Sixth Ed., Elsevier, 2013).

[45] Keang-Po Ho, J.M. Kahn, Linear Propagation Effects in Mode-Division Multiplexing Systems, J. Lightwave Tech. 32, 4 (2014).

[46] D. Psaltis, C. Moser, Imaging with multimode fibers, Opt. and Photon. News 27, 24 (2016).

[47] W. Xiong, C.W. Hsu, Y. Bromberg, J.E. AntonioLopez, R.A. Correa, and H. Cao, Complete polarization control in multimode fibers with polarization and mode coupling, Light: Science \& Applications 7:54 (2018).

[48] P.K.A. Wai, C.R. Menyuk, Polarization mode dispersion, decorrelation, and diffusion in optical fibers with randomly varying birefringence, IEEE J. Lightw. Technol. 14, 148 (1996).

[49] A. Mecozzi, C. Antonelli, M. Shtaif, Nonlinear propagation in multimode fibers in the strong coupling regime, Opt. Exp. 20, 11673 (2012).

[50] A. Mecozzi, C. Antonelli, M. Shtaif, Coupled Manakov equations in multimode fibers with strongly coupled groups of modes, Opt. Exp. 20, 23436 (2012).

[51] S. Mumtaz, R.J. Essiambre, G.P. Agrawal, Nonlinear propagation in multimode and multicore fibers: Generalization of the Manakov equations, J. Lightw. Technol. 31, 398 (2013).

[52] Y. Xiao, R.-J. Essiambre, M. Desgroseilliers, A.M. Tulino, R. Ryf, S. Mumtaz, G.P. Agrawal, Theory of intermodal four-wave mixing with random linear mode coupling in few-mode fibers, Opt. Exp. 22, 32039 (2014).

[53] S. Buch, S. Mumtaz, R.-J. Essiambre, A.M. Tulino, G.P. Agrawal, Averaged nonlinear equations for multimode fibers valid in all regimes of random linear coupling Opt. Fiber Techn. 48, 123 (2019).

[54] K. Krupa, G.G. Castaneda, A. Tonello, A. Ninag, D.S. Kharenko, M. Fabert, V. Couderc, G. Millot, U. Minoni, D. Modotto, S. Wabnitz, Nonlinear polarization dynamics of Kerr beam self-cleaning in a graded-index multimode optical fiber, Opt. Lett. 44, 171 (2019).

[55] E.A. Kartashova, Wave resonances in systems with discrete spectra. In: V.E. Zakharov, (ed.) Nonlinear Waves and Weak Turbulence, p. 95. Springer, Berlin (1998).

[56] V.E. Zakharov, A.O. Korotkevich, A.N. Pushkarev, A.I. Dyachenko, Mesoscopic Wave Turbulence, JETP Letters 82, 487 (2005).

[57] S.V. Nazarenko, Sandpile behaviour in discrete waterwave turbulence, J. Stat. Mech. L02002, (2006).

[58] E. Kartashova, S. Nazarenko, and O. Rudenko, Resonant interactions of nonlinear water waves in a finite basin, Phys. Rev. E 78, 016304 (2008).

[59] E. Kartashova, Discrete wave turbulence, EPL 87, 44001 (2009).

[60] E.A. Kartashova, Nonlinear Resonance Analysis Theory, Computation, Applications (Cambridge University Press, Cambridge, 2010)

[61] V.S. L'vov, S.V. Nazarenko, Discrete and mesoscopic regimes of finite-size wave turbulence, Phys. Rev. E 82, 056322 (2010).

[62] J. Harris, C. Connaughton, M.D. Bustamante, Percolation transition in the kinematics of nonlinear resonance broadening in Charney-Hasegawa-Mima model of Rossby wave turbulence, New Journal of Physics 15, 083011 (2013).

[63] K.L. Harper, M.D. Bustamante, S.V. Nazarenko, Quadratic invariants for discrete clusters of weakly interacting waves, J. Phys. A: Math. Theor. 46, 245501 (2013).

[64] M. Bustamante, B. Quinn, D. Lucas, Robust Energy Transfer Mechanism via Precession Resonance in Nonlinear Turbulent Wave Systems, Phys. Rev. Lett. 113, 084502 (2014).

[65] S. Kuksin, A. Maiocchi, The Effective Equation Method, in New approaches to nonlinear waves, edited by E. Tobisch (Springer, LNP 908, page 20).

[66] R. Hassaini, N. Mordant, Confinement effects on gravity-capillary wave turbulence, Phys. Rev. Fluids 3, 094805 (2018).

[67] E. Deliancourt, M. Fabert, A. Tonello, K. Krupa, A. Desfarges-Berthelemot, V. Kermene, G. Millot, A. Barthélémy, S. Wabnitz, V. Couderc, Kerr beam selfcleaning on the $\mathrm{LP}_{11}$ mode in graded-index multimode fibers, OSA Continuum 2, 1089 (2019).

[68] Z. Liu, L.G. Wright, D.N. Christodoulides, F.W. Wise, Kerr self-cleaning of femtosecond-pulsed beams in graded-index multimode fiber, Optics Letters 413675 (2016).

[69] W. H. Renninger and F. W. Wise, Optical solitons in graded-index multimode fibres, Nature Commun. 4, 1719 (2013).

[70] L.G. Wright, W.H. Renninger, D.N. Christodoulides, F.W. Wise, Spatiotemporal dynamics of multimode optical solitons Opt. Express 23, 3492-3506 (2015).

[71] L.G. Wright, S. Wabnitz, D.N. Christodoulides, F.W. Wise, Ultrabroadband dispersive radiation by spatiotemporal oscillation of multimode waves, Phys. Rev. Lett. 115, 223902 (2015).

[72] L. Wright, D. N. Christodoulides, and F. W. Wise, Controllable spatiotemporal nonlinear effects in multimode fibres, Nat. Photonics 9, 306 (2015).

[73] M. Conforti, C. Mas Arabi, A. Mussot, A. Kudlinski, Fast and accurate modeling of nonlinear pulse propaga- 
tion in graded-index multimode fibers, Opt. Lett. 42, 4004 (2017).

[74] A.S. Ahsan, G.P. Agrawal, Graded-index solitons in multimode fibers, Opt. Lett. 43, 3345 (2018)

[75] P. Horak, F. Poletti, Multimode nonlinear fibre optics: Theory and applications, in Recent Progress in Optical Fiber Research, M. Yasin, S. W. Harun, and H. Arof, eds. (InTech, 2012), 3-25.

[76] F. Poletti and P. Horak, Description of ultrashort pulse propagation in multimode optical fibers, J. Opt. Soc. Am. B 25, 1645 (2008).

[77] G. Agrawal, Nonlinear Fiber Optics, fifth Ed., Academic Press, New York, 2012

[78] The Fermi-Pasta-Ulam Problem: A Status Report, edited by G. Gallavotti (Springer, New York, 2007).

[79] G. Benettin, H. Christodoulidi, and A. Ponno, The Fermi-Pasta-Ulam Problem and Its Underlying Integrable Dynamics, J. Stat. Phys. 152, 195 (2013).

[80] M. Onorato, L. Vozella, D. Proment, and Y. V. L'vov, Route to Thermalization in the $\alpha$-Fermi-Pasta-Ulam System, Proc. Natl. Acad. Sci. U.S.A. 112, 4208 (2015).

[81] Y.V. Lvov and M. Onorato, Double Scaling in the Relaxation Time in the Fermi-Pasta-Ulam-Tsingou Model, Phys. Rev. Lett. 120, 144301 (2018).

[82] M. Guasoni, J. Garnier, B. Rumpf, D. Sugny, J. Fatome, F. Amrani, G. Millot, and A. Picozzi, Incoherent FermiPasta-Ulam Recurrences and Unconstrained Thermalization Mediated by Strong Phase Correlations, Phys. Rev. X 7, 011025 (2017).

[83] L. Ermann, D.L. Shepelyansky, Quantum Gibbs distribution from dynamical thermalization in classical nonlinear lattices, New Journal of Physics 15, 123004 (2013).

[84] L. Ermann, E. Vergini, D.L. Shepelyansky, Dynamical thermalization of Bose-Einstein condensate in Bunimovich stadium, Europhys. Lett. 111, 50009 (2015).

[85] N. Cherroret, T. Karpiuk, B. Grémaud, C. Miniatura, Thermalization of matter waves in speckle potentials, Phys. Rev. A 92, 063614 (2015).

[86] S. Chibbaro, G. Dematteis, C. Josserand, and L. Rondoni, Wave-turbulence theory of four-wave nonlinear interactions, Phys. Rev. E 96, 021101(R) (2017).

[87] S. Chibbaro, G. Dematteis, L. Rondoni, 4-wave dynamics in kinetic wave turbulence, Physica D 362, 24 (2018).

[88] C. Antonelli, A. Mecozzi, M. Shtaif, P.J. Winzer, Stokes-space analysis of modal dispersion in fibers with multiple mode transmission, Opt. Exp. 20, 11718 (2012).

[89] A. Galtarossa, L. Palmieri, M. Schiano, T. Tambosso, Measurement of birefringence correlation length in long single-mode fibers, Opt. Lett. 26, 962 (2001).

[90] E. Deliancourt, M. Fabert, A. Tonello, K. Krupa, A. Desfarges-Berthelemot, V. Kermene, G. Millot, A. Barthélémy, S. Wabnitz, V. Couderc, Wavefront shaping for optimized many-mode Kerr beam self-cleaning in graded-index multimode fiber, Opt. Express 12, 1731117321 (2019).

[91] H. Frostig, E. Small, A. Daniel, P. Oulevey, S. Derevyanko, Y. Silberberg, Focusing light by wavefront shaping through disorder and nonlinearity, Optica 4, 1073 (2017).

[92] M.B. Shemirani, W. Mao, R.A. Panicker, and J.M. Kahn, Principal modes in graded-index multimode fiber in presence of spatial- and polarization-mode coupling,
J. Lightw. Tech. 27, 1248 (2009).

[93] X. Shen, J.M. Kahn, and M.A. Horowitz, Compensation for multimode fiber dispersion by adaptive optics, Optics Letters 30, 2985 (2005).

[94] R. Dupiol, K. Krupa, A. Tonello, M. Fabert, D. Modotto S. Wabnitz, G. Millot, and V. Couderc, Interplay of Kerr and Raman beam cleaning with a multimode microstructure fiber, Opt. Letters 43, 587 (2018).

[95] L.G. Wright, D.N. Christodoulides, F.W. Wise, Spatiotemporal mode-locking in multimode fiber lasers, Science 358, 94 (2017).

[96] J. Laegsgaard, Spatial beam cleanup by pure Kerr processes in multimode fibers, Opt. Lett. 43, 2700 (2018).

[97] O.V. Shtyrina, M.P. Fedoruk, Y.S. Kivshar, and S.K. Turitsyn, Coexistence of collapse and stable spatiotemporal solitons in multimode fibers, Phys. Rev. A 97, 013841 (2018).

[98] C. Mas Arabi, A. Kudlinski, A. Mussot, and M. Conforti, Geometric parametric instability in periodically modulated graded-index multimode fibers, Phys. Rev. A 97, 023803 (2018).

[99] J. Garnier, G. Xu, S. Trillo and A. Picozzi, Incoherent Dispersive Shocks in the Spectral Evolution of Random Waves, Phys. Rev. Lett 111, 113902 (2013).

[100] G. Xu, D. Vocke, D. Faccio, J. Garnier, T. Rogers, S. Trillo, A. Picozzi, From coherent shocklets to giant collective incoherent shock waves in nonlocal turbulent flows, Nature Comm. 6, 8131 (2015).

[101] A. Eftekhar, L.G. Wright, M.S. Mills, M. Kolesik, R. Amezcua Correa, F.W. Wise, and D.N. Christodoulides, Versatile supercontinuum generation in parabolic multimode optical fibers, Opt. Exp. 25, 9078-9087 (2017).

[102] K. Krupa, C. Louot, V. Couderc, M. Fabert, R. Guenard, B.M. Shalaby, A. Tonello, D. Pagnoux, P. Leproux, A. Bendahmane, R. Dupiol, G. Millot, S. Wabnitz, Spatiotemporal characterization of supercontinuum extending from the visible to the mid-infrared in a multimode graded-index optical fiber, Opt. Lett. 41, 57855788 (2016).

[103] G. Lopez-Galmiche, Z. Sanjabi Eznaveh, M. A. Eftekhar, J. Antonio Lopez, L. G. Wright, F. Wise, D. Christodoulides, and R. Amezcua Correa, Visible supercontinuum generation in a graded index multimode fiber pumped at 1064nm, Opt. Lett. 41, 2553-2556 (2016).

[104] B. Barviau, B. Kibler, and A. Picozzi, Wave-turbulence approach of supercontinuum generation: Influence of self-steepening and higher-order dispersion, Phys. Rev. A 79, 063840 (2009).

[105] B. Barviau, J. Garnier, G. Xu, B. Kibler, G. Millot, and A. Picozzi, Truncated thermalization of incoherent optical waves through supercontinuum generation in photonic crystal fibers, Phys. Rev. A 87, 035803 (2013)

[106] G. Xu, J. Garnier, B. Rumpf, A. Fusaro, P. Suret, S. Randoux, A. Kudlinski, G. Millot, and A. Picozzi, Origins of spectral broadening of incoherent waves: Catastrophic process of coherence degradation, Phys. Rev. A 96, 023817 (2017).

[107] V.M. Malkin and N.J. Fisch, Transition between inverse and direct energy cascades in multiscale optical turbulence, Phys. Rev. E 97, 032202 (2018).

[108] G. Labeyrie, T. Ackemann, B. Klappauf, M. Pesch, G.L. Lippi, and R. Kaiser, Nonlinear beam shaping by a cloud of cold Rb atoms, Eur. Phys. J. D 22, 473 (2003).

[109] D. Vocke, K. Wilson, F. Marino, I. Carusotto, E.M. 
Wright, T. Roger, B.P. Anderson, P. Öhberg, D. Faccio, Role of geometry in the superfluid flow of nonlocal photon fluids, Phys. Rev. A 94, 013849 (2016).

[110] C. Michel, O. Boughdad, M. Albert, P.-E. Larré, M. Bellec, Superfluid motion and drag-force cancellation in a fluid of light, Nature Comm. 9, 2108 (2018).

[111] Q. Fontaine, T. Bienaimé, S. Pigeon, E. Giacobino, A. Bramati, and Q. Glorieux, Observation of the Bogoliubov Dispersion in a Fluid of Light, Phys. Rev. Lett. 121, 183604 (2018).

[112] J. Berges, S. Borsanyi, and C. Wetterich, Prethermalization, Phys. Rev. Lett. 93, 142002 (2004).

[113] T. Langen, T. Gasenzer, and J. Schmiedmayer, Prethermalization and universal dynamics in near-integrable quantum systems, J. Stat. Mech. 6, 064009 (2016).

[114] P.-E. Larré, I. Carusotto, Prethermalization in a quenched one-dimensional quantum fluid of light, Eur. Phys. J. D 70: 45 (2016).

[115] P.-E. Larré, D. Delande, N. Cherroret, Postquench prethermalization in a disordered quantum fluid of light, Phys. Rev. A 97, 043805 (2018).

[116] A. Campa, T. Dauxois, S. Ruffo, Statistical mechanics and dynamics of solvable models with long-range interactions, Physics Reports, 480, 57 (2009).

[117] S. Schütz, G. Morigi Prethermalization of Atoms Due to Photon-Mediated Long-Range Interactions, Phys. Rev. Lett. 113, 203002 (2014).

[118] A. Picozzi and J. Garnier, Incoherent Soliton Turbulence in Nonlocal Nonlinear Media, Phys. Rev. Lett. 107, 233901 (2011).

[119] P. Suret, A. Picozzi, and S. Randoux, Wave turbulence in integrable systems: nonlinear propagation of incoherent optical waves in single-mode fibers, Optics Express 19, 17852 (2011).

[120] S. Randoux, P. Walczak, M. Onorato, P. Suret, Nonlinear random optical waves: Integrable turbulence, rogue waves and intermittency, Physica D 333, 323 (2016).

[121] M. Gring, M. Kuhnert, T. Langen, T. Kitagawa, B.
Rauer, M. Schreitl, I. Mazets, D. Adu Smith, E. Demler, J. Schmiedmayer, Relaxation and prethermalization in an isolated quantum system, Science, 337, 1318 (2012).

[122] T. Langen, T. Gasenzer, J. Schmiedmayer, Prethermalization and universal dynamics in near-integrable quantum systems, J. Stat. Mech. 6, 064009 (2016).

[123] I. Chantesana, A. Piñeiro Orioli, and T. Gasenzer, Kinetic theory of nonthermal fixed points in a Bose gas Phys. Rev. A 99, 043620 (2019).

[124] Y. Lahini, A. Avidan, F. Pozzi, M. Sorel, R. Morandotti, D.N. Christodoulides, Y. Silberberg, Anderson Localization and nonlinearity in one-dimensional disordered photonic lattices, Phys. Rev. Lett. 100, 013906 (2008).

[125] M. Segev, Y. Silberberg, D.N. Christodoulides, Anderson localization of light, Nature Photonics 7, 197 (2013).

[126] M. Leonetti, S. Karbasi, A. Mafi, C. Conti, Light focusing in the Anderson regime, Nature Comm. 5, 4534 (2014).

[127] W. Schirmacher, B. Abaie, A. Mafi, G. Ruocco, M. Leonetti, What is the right theory for Anderson localization of light? An experimental test, Phys. Rev. Lett. 120, 067401 (2018).

[128] D. Pierangeli, A. Tavani, F. Di Mei, A.J. Agranat, C. Conti, E. DelRe, Observation of replica symmetry breaking in disordered nonlinear wave propagation, $\mathrm{Na}-$ ture Commun. 8, 1501 (2017).

[129] C. Conti and L. Leuzzi, Complexity of waves in nonlinear disordered media, Phys. Rev. B 83, 134204 (2011).

[130] F. Antenucci, M. Ibanez Berganza, L. Leuzzi, Statistical physics of nonlinear wave interaction, Phys. Rev. B 92, 014204 (2015).

[131] V. V. Konotop and L. Vazquez, Nonlinear Random Waves (World Scientific, Singapore, 1994).

[132] J. Kurzweil and J. Jarník, Limit processes in ordinary differential equations, Zeitschrift für angewandte Mathematik und Physik ZAMP 38, 241-256 (1987). 